\title{
Irregular Trellis for the Near-Capacity Unary Error Correction Coding of Symbol Values From an Infinite Set
}

\author{
Wenbo Zhang, Student Member, IEEE, Matthew F. Brejza, Tao Wang, Robert G. Maunder, Senior Member, IEEE, \\ and Lajos Hanzo, Fellow, IEEE
}

Abstract-Irregular joint source and channel coding (JSCC) scheme is proposed, which we refer to as the irregular unary error correction (IrUEC) code. This code operates on the basis of a single irregular trellis, instead of employing a set of separate regular trellises, as in previous irregular trellis-based codes. Our irregular trellis is designed with consideration of the UEC free distance, which we characterize for the first time in this paper. We conceive the serial concatenation of the proposed IrUEC code with an irregular unity rate code (IrURC) code and propose a new EXtrinsic Information Transfer (EXIT) chart matching algorithm for parametrizing these codes. This facilitates the creation of a narrow EXIT tunnel at a low $E_{\mathrm{b}} / N_{0}$ value and provides near-capacity operation. Owing to this, our scheme is found to offer a low symbol error ratio (SER), which is within $0.4 \mathrm{~dB}$ of the discrete-input continuous-output memoryless channel (DCMC) capacity bound in a particular practical scenario, where gray-mapped quaternary phase shift keying (QPSK) modulation is employed for transmission over an uncorrelated narrowband Rayleigh-fading channel with an effective throughput of $0.508 \mathrm{bits}^{-1} \mathrm{~Hz}^{-1}$. Furthermore, the proposed IrUEC-IrURC scheme offers a SER performance gain of $0.8 \mathrm{~dB}$, compared to the best of several regular and irregular separate source and channel coding (SSCC) benchmarkers, which is achieved without any increase in transmission energy, bandwidth, transmit duration, or decoding complexity.

Index Terms-Joint source-channel coding, irregular codecs, channel capacity, iterative decoding.

\section{INTRODUCTION}

N MOBILE wireless scenarios, multimedia transmission is required to be bandwidth efficient and resilient to transmission errors, motivating both source and channel coding [1]-[3]. Classic Separate Source and Channel Coding (SSCC) may be achieved by combining a near-entropy source code with a near-capacity channel code. In this scenario, it is theoretically

Manuscript received October 31, 2014; revised April 14, 2015 and July 25, 2015; accepted October 8, 2015. This work was supported in part by the EPSRC, Swindon UK under Grant EP/J015520/1 and Grant EP/L010550/1, in part by the TSB Swindon UK under Grant TS/L009390/1, in part by the RCUK under the India-UK Advanced Technology Centre (IU-ARC), and in part by the EU under the CONCERTO project and in part by the European Research Council's Advanced Fellow grant. The associate editor coordinating the review of this paper and approving it for publication was M. Xiao.

The authors are with School of Electronics and Computer Science, University of Southampton, Southampton SO17 1BJ, U.K. (e-mail: wz4g11@ecs.soton. ac.uk; mfb2g09@ecs.soton.ac.uk; tw08r@ecs.soton.ac.uk; rm@ecs.soton. ac.uk; lh@ecs.soton.ac.uk).

Color versions of one or more of the figures in this paper are available online at http://ieeexplore.iee.org.

Digital Object Identifier 10.1109/TCOMM.2015.2493149 possible to reconstruct the source information with an infinitesimally low probability of error, provided that the transmission rate does not exceed the channel's capacity [4]. However, separate source-channel coding [4] is only capable of approaching the capacity in the general case by imposing both infinite complexity and infinite latency. For example, adaptive arithmetic coding [5] and Lempel-Ziv coding [6] are capable of encoding a sequence of symbols using a near-entropy number of bits per symbol. However, these schemes require both the transmitter and receiver to accurately estimate the occurrence probability of every symbol value that the source produces. In practice, the occurrence probability of rare symbol values can only be accurately estimated, if a sufficiently large number of symbols has been observed, hence potentially imposing an excessive latency.

This motivates the design of universal codes, such as the Elias Gamma (EG) code [7], which facilitate the binary encoding of symbols selected from infinite sets, without requiring any knowledge of the corresponding occurrence probabilities at either the transmitter or receiver. The H.264 video codec [8] employs the EG code and this may be concatenated with classic channel codes, such as a Convolutional Code (CC) to provide a separate error correction capability. Nevertheless, this SSCC typically suffers from a capacity loss, owing to the residual redundancy that is typically retained during EG encoding, which results in an average number of EG-encoded bits per symbol that exceeds the entropy of the symbols.

In order to exploit the residual redundancy and hence to achieve near-capacity operation, the classic SSCC schemes may be replaced by Joint Source and Channel Coding (JSCC) arrangements [9] in many applications. As we have previously demonstrated in [10, Fig. 1], the symbols that are EG encoded in H.264 are approximately zeta probability distributed [11], resulting in most symbols having low values, but some rare symbols having values around 1000 . Until recently, the decoding complexity of all previous JSCCs, such as Reversible Variable Length Codes (RVLCs) [12] and Variable Length Error Correction (VLEC) codes [13], increased rapidly with the cardinality of the symbol set, so much so that it became excessive for the H.264 symbol probability distribution and asymptotically tending to infinity, when the cardinality is infinite.

Against this background, a novel JSCC scheme referred to as a Unary Error Correction (UEC) code [10] was proposed as the first JSCC that mitigates the capacity loss and incurs 

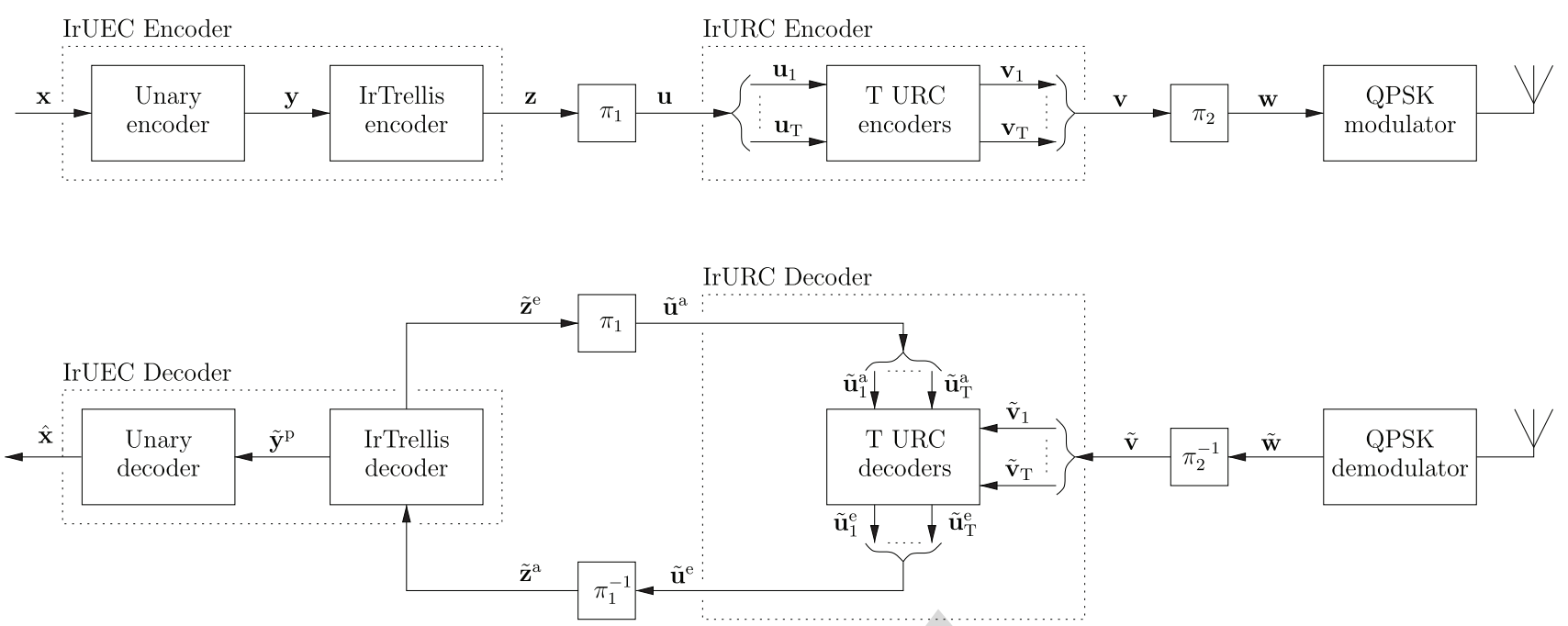

Fig. 1. Schematic of the proposed IrUEC-IrURC scheme, in which an IrUEC code is serially concatenated with IrURC code and Gray-coded QPSK modulation schemes. Here, $\pi_{1}$ and $\pi_{2}$ represent interleavers, while $\pi_{1}^{-1}$ and $\pi_{2}^{-1}$ represent the corresponding deinterleavers.

only a moderate decoding complexity, even when the cardinality of the symbol set is infinite. In a particular practical scenario, an iteratively-decoded serial concatenation of the UEC code with an Irregular Unity Rate Code (IrURC) was shown to offer a $1.3 \mathrm{~dB}$ gain compared to a SSCC benchmarker, without incurring an increased transmission energy, duration, bandwidth or decoding complexity. Furthermore, this was achieved within $1.6 \mathrm{~dB}$ of the Quaternary Phase Shift Keying (QPSK)-modulated uncorrelated narrow band Rayleigh fading Discrete-input Continuous-output Memoryless Channel (DCMC) capacity bound.

In this paper, we will further exploit the properties of UEC codes in order to facilitate reliable operation even closer to the capacity bound. More specifically, we propose an Irregular Unary Error Correction (IrUEC) code, which extends the regular UEC of our previous work [10]. This IrUEC code employs different UEC parametrizations for the coding of different subsets of each message frame, in analogy with previous irregular codes, such as the IrURC [14], the Irregular Convolutional Code (IrCC) [15] and the Irregular Variable Length Code (IrVLC) [16]. However, these previous irregular codes operate on the basis of a number of separate trellises, each of which has a different but uniform structure and is used for the coding of a different subset of the message frame. By contrast, our new IrUEC code operates on the basis of a single irregular trellis having a novel design. This trellis has a non-uniform structure that applies different UEC parametrizations for different subsets of the frame on a bit-by-bit basis. This allows the irregularity of the proposed IrUEC code to be controlled on a fine-grained bit-by-bit basis, rather than on a symbol-bysymbol basis, hence facilitating nearer-to-capacity operation. More specifically, our results demonstrate that controlling the IrUEC irregularity on a bit-by-bit basis offers gains of up to $0.2 \mathrm{~dB}$ over the symbol-by-symbol approach, without imposing any increase in transmission energy, bandwidth, latency or decoding complexity.

This bit-by-bit IrUEC approach is facilitated by some particular properties of UEC codes, which grant some commonality to all UEC parametrizations. By exploiting this fine-grained 117 control of the IrUEC irregularity, the IrUEC EXtrinsic 118 Information Transfer (EXIT) function may be shaped to cre- 119 ate a narrow, but marginally open EXIT chart tunnel. This 120 implies that near-capacity operation is facilitated, according to 121 the theoretical properties of EXIT charts [17].

The rest of this paper is organized as follows. Section II 123 describes a transmitter that serially concatenates the proposed 124 IrUEC encoder with a IrURC encoder, while Section III 125 describes the corresponding iterative receiver. The IrUEC 126 encoder and decoder operate on the basis of our novel irregular 127 trellis structure, which allows bit-level control of the irregular 128 coding fractions. The free distance of UEC codes is quantified 129 for the first time in Section IV, which proposes a novel low- 130 complexity heuristic method conceived for this purpose. This is 131 used for selecting a family of UEC trellis structures having a 132 wide variety of EXIT function shapes. The resultant UEC trel- 133 lis family maximises the design freedom for the IrUEC EXIT 134 function and therefore has a general applicability for IrUEC 135 codes used in diverse applications. Furthermore, for any partic- 136 ular application of an IrUEC code, we propose a double-sided 137 EXIT chart matching algorithm for selecting the specific frac- 138 tion of the frame that should be encoded using each IrUEC 139 and IrURC trellis structure. This allows the EXIT functions of 140 IrUEC and IrURC codes to be accurately shaped for closely 141 matching each other, hence creating a narrow but marginally 142 open EXIT chart tunnel. In Section V, the proposed IrUEC- 143 IrURC scheme is compared to an irregular JSCC benchmarker, 144 which is referred to as the EG-IrCC-IrURC scheme. The first 145 version of this benchmarker employs the recursive systematic 146 CCs that were originally recommended as IrCC component 147 codes in [15]. However, we demonstrate that the systematic 148 nature of these IrCC component codes results in a capacity loss. 149 This motivates the employment of the second version of our 150 EG-IrCC-IrURC benchmarker, which employs the recursive 151 non-systematic CCs of [10] as the IrCC component codes. The 152 simulation results of Section V show that in a particular prac- 153 tical scenario, the proposed IrUEC-IrURC scheme provides a 154 
$0.8 \mathrm{~dB}$ gain over the best SSCC benchmarker, while operating within $0.4 \mathrm{~dB}$ of the capacity bound. This is achieved without any increase in transmission energy, bandwidth, latency or decoding complexity. Finally, Section VI concludes the paper.

\section{IRUEC-IRURC ENCODER}

In this section, we introduce the transmitter of the proposed IrUEC-IrURC scheme of Fig. 1. The IrURC encoder employs $T$ number of component Unity Rate Code (URC) encoders $\left\{\mathrm{URC}^{t}\right\}_{t=1}^{T}$, each having a distinct independent trellis structure. By contrast, the IrUEC employs a unary encoder and a novel Irregular Trellis (IrTrellis) encoder with a single irregular trellis. However, in analogy with the IrURC code, we note that this irregular trellis comprises a merging of $S$ component UEC trellis structures $\left\{\mathrm{UEC}^{S}\right\}_{s=1}^{S}$, where $\mathrm{UEC}^{S}$ is the $s$-th component UEC trellis structure that is defined by the corresponding codeword set $\mathbb{C}_{s}$, as illustrated in [10, Fig. 3(a)]. In Section II-A and Section II-B, the two components of the IrUEC encoder in Fig. 1, namely the unary encoder and the novel IrTrellis encoder are detailed. The IrURC encoder and the modulator are introduced in Section II-C.

\section{A. Unary Encoder}

The IrUEC encoder is designed for conveying a vector $\mathbf{x}=\left[x_{i}\right]_{i=1}^{a}$ comprising $a$ number of symbols, as shown in Fig. 1. The value of each symbol $x_{i} \in \mathbb{N}_{1}$ may be modeled by an Independent and Identically Distributed (IID) Random Variable $(\mathrm{RV}) X_{i}$, which adopts the value $x$ with a probability of $\operatorname{Pr}\left(X_{i}=x\right)=P(x)$, where $\mathbb{N}_{1}=\{1,2,3, \ldots, \infty\}$ is the infinite-cardinality set comprising all positive integers. Throughout this paper we assume that the symbol values obey a zeta probability distribution [11], since this models the symbols produced by multimedia encoders, as described in Section I. The zeta probability distribution is defined as

$$
P(x)=\frac{x^{-s}}{\zeta(s)},
$$

where $\zeta(s)=\sum_{x \in \mathbb{N}_{1}} x^{-s}$ is the Riemann zeta function, $s>1$ parametrizes the zeta distribution and $p_{1}=\operatorname{Pr}\left(X_{i}=1\right)=$ $1 / \zeta(s)$ is the probability of occurrence for the most frequently occurring symbol value, namely $x=1$. Without loss of generality, Table I exemplifies the first ten symbol probabilities $P\left(x_{i}\right)$ for a zeta distribution having the parameter $p_{1}=0.797$, which corresponds to $s=2.77$ and was found in [10] to allow a fair comparison between unary- and EG-based schemes. Note that other $p_{1}$ values of $0.694,0.8$ and 0.9 have been investigated in [18], [19]. In the situation where the symbols obey the zeta probability distribution of (1), the symbol entropy is given by

$$
H_{X}=\sum_{x \in \mathbb{N}_{1}} H[P(x)]=\frac{\ln (\zeta(s))}{\ln (2)}-\frac{s \zeta^{\prime}(s)}{\ln (2) \zeta(s)},
$$

where $H[p]=p \log _{2}(1 / p)$ and $\zeta^{\prime}(s)=-\sum_{x \in \mathbb{N}_{1}} \ln (x) x^{-s}$ is the derivative of the Riemann zeta function.

As shown in Fig. 1, the IrUEC encoder represents the source vector $\mathbf{x}$ using a unary encoder. More specifically, each symbol
TABLE I

The First Ten Symbol Probabilities For a Zeta Distribution HAVING THE PARAMETER $p_{1}=0.797$, AS Well AS THE CORRESPONDING UNARY AND EG CODEWORDS

\begin{tabular}{|c|l|l|l|}
\hline \multirow{2}{*}{$x_{i}$} & \multirow{2}{*}{$P\left(x_{i}\right)$} & \multicolumn{2}{|c|}{$\mathrm{y}_{i}$} \\
\cline { 3 - 4 } & & \multicolumn{1}{|c|}{ Unary } & EG \\
\hline 1 & 0.797 & 0 & 1 \\
\hline 2 & 0.117 & 10 & 010 \\
\hline 3 & 0.038 & 110 & 011 \\
\hline 4 & 0.017 & 1110 & 00100 \\
\hline 5 & 0.009 & 11110 & 00101 \\
\hline 6 & 0.006 & 111110 & 00110 \\
\hline 7 & 0.004 & 1111110 & 00111 \\
\hline 8 & 0.003 & 11111110 & 0001000 \\
\hline 9 & 0.002 & 111111110 & 0001001 \\
\hline 10 & 0.001 & 1111111110 & 0001010 \\
\hline
\end{tabular}

$x_{i}$ in the vector $\mathbf{x}$ is represented by a corresponding codeword 202 $\mathbf{y}_{i}$ that comprises $x_{i}$ bits, namely $\left(x_{i}-1\right)$ binary ones followed 203 by a zero, as exemplified in Table I. When the symbols adopt 204 the zeta distribution of (1), the average unary codeword length $l 205$ is only finite for $s>2$ and hence for $p_{1}>0.608$ [10], in which 206 case we have

$$
l=\sum_{x \in \mathbb{N}_{1}} P(x) \cdot x=\frac{\zeta(s-1)}{\zeta(s)} .
$$

Note that for $p_{1} \leq 0.608$, our Elias Gamma Error Correction 208 (EGEC) code of [19] may be employed in order to achieve a 209 finite average codeword length, albeit at the cost of an increased 210 complexity. In our future work, we will consider a novel 211 Irregular EGEC code, which has a finite codeword length for 212 $p_{1} \leq 0.608$. Without loss of generality, in the example scenario 213 of $p_{1}=0.797$, an average codeword length of $l=1.54$ results. 214 The output of the unary encoder is generated by concatenating 215 the selected codewords $\left\{\mathbf{y}_{i}\right\}_{i=1}^{a}$, in order to form the $b$-bit vec- 216 tor $\mathbf{y}=\left[y_{j}\right]_{j=1}^{b}$. For example, the source vector $\mathbf{x}=[4,1,2]$ of 217 $a=3$ symbols yields the $b=7$-bit vector $\mathbf{y}=[1110010]$. Note 218 that the average length of the bit vector $\mathbf{y}$ is given by $(a \cdot l)$.

\section{B. IrTrellis Encoder}

Following unary encoding, the IrTrellis encoder of Fig. 1221 employs a single new irregular trellis to encode the bit vec- 222 tor $\mathbf{y}$, rather than using a selection of separate trellis structures, 223 as is necessary for the IrCC [15], IrVLC [16] and IrURC [14] 224 coding schemes. Our novel irregular trellis structure is facil- 225 itated by the properties of the generalised trellis structure of 226 [10, Fig. 3(a)], which was the basis of our previous work on 227 regular UEC codes. This trellis structure is parametrized by 228 an even number of states $r$ and by the UEC codeword set $\mathbb{C}, 229$ which comprises $r / 2$ binary codewords of a particular length 230 $n$. Each bit $y_{j}$ of the unary-encoded bit sequence $\mathbf{y}=\left[y_{j}\right]_{j=1}^{b} 231$ corresponds to a transition in the UEC trellis from the previous 232 state $m_{j-1} \in\{1,2, \ldots, r\}$ to the next state $m_{j} \in\{1,2, \ldots, r\} .233$ Each next state $m_{j}$ is selected from two legitimate alternatives, 234 depending both on the previous state $m_{j-1}$ and on the bit value 235 $y_{j}$, according to [18, (3)]. More specifically, regardless of how 236 the UEC trellis is parametrized, a unary-coded bit of $y_{j}=1237$ causes a transition towards state $m_{j}=r-1$ or $r$ of the gener- 238 alised UEC trellis of [10, Fig. 3(a)], while the $y_{j}=0$-valued bit 239 
at the end of each unary codeword causes a transition to state $m_{j}=1$ or 2 , depending on whether the current symbol $x_{i}$ has an odd or even index $i$.

This common feature of all UEC trellises maintains synchronisation with the unary codewords and allows the residual redundancy that remains following unary encoding to be explicated for error correction. Furthermore, this common treatment of the unary-encoded bits in $\mathbf{y}$ between all UEC trellises allows them to merge in order to form our novel irregular trellis. More specifically, our novel irregular trellis can be seen as concatenation of a number of individual UEC trellis structures with different numbers of states $r$ and different codeword sets $\mathbb{C}$. By contrast, CCs, Variable Length Codes (VLCs) and URC codes having different parametrizations do not generally exhibit the required similarity in their trellises. More specifically, the final state of a particular component encoder has no specific relationship with the initial state of the subsequent component encoder, hence preventing their amalgamation into IrCC, IrVLC and IrURC trellises, respectively.

The IrTrellis encoder of Fig. 1 encodes the $b$-bit unaryencoded bit sequence $\mathbf{y}=\left[y_{j}\right]_{j=1}^{b}$ using an irregular trellis that is obtained by concatenating $b$ number of regular UEC trellis structures. The proposed IrTrellis can be constructed using diverse combinations of component regular UEC trellises, having any parametrization. However, the component regular trellises may be strategically selected in order to carefully shape the EXIT function of the IrUEC code, for the sake of producing a narrow EXIT chart tunnel and for facilitating near-capacity operation, as it will be detailed in Section IV. Without loss of generality, Fig. 1 provides an example of the irregular trellis for the example scenario where we have $b=7$. Each bit $y_{j}$ in the vector $\mathbf{y}$ is encoded using the corresponding one of these $b$ trellis structures, which is parametrized by an even number of states $r_{j}$ and the codeword set $\mathbb{C}_{j}=$ $\left\{\mathbf{c}_{1}^{j}, \mathbf{c}_{2}^{j}, \ldots, \mathbf{c}_{r_{j} / 2-1}^{j}, \mathbf{c}_{r_{j} / 2}^{j}\right\}$, which comprises $r_{j} / 2$ binary codewords of a particular length $n_{j}$. Note that successive trellis structures can have different numbers of states, subject to the constraint $r_{j} \leq r_{j-1}+2$, as it will be demonstrated in the following discussions. Note that this constraint does not restrict the generality of the IrUEC trellis, since the IrUEC EXIT function shape is independent of the ordering of the component trellis structures.
As in the regular UEC trellis of [10], the encoding process 282 always emerges from the state $m_{0}=1$. The unary-encoded 283 bits of $\mathbf{y}$ are considered in order of increasing index $j$ and 284 each bit $y_{j}$ causes the novel IrTrellis to traverse from the 285 previous state $m_{j-1} \in\left\{1,2, \ldots, r_{j-1}\right\}$ to the next state $m_{j} \in 286$ $\left\{1,2, \ldots, r_{j}\right\}$, which is selected from two legitimate alterna- 287 tives. More specifically,

$$
m_{j}=\left\{\begin{array}{ll}
1+\operatorname{odd}\left(m_{j-1}\right) & \text { if } y_{j}=0 \\
\min \left[m_{j-1}+2, r_{j}-\operatorname{odd}\left(m_{j-1}\right)\right] & \text { if } y_{j}=1
\end{array},\right.
$$

where the function odd $(\cdot)$ yields 1 if the operand is odd or 0 if 289 it is even. Note that the next state $m_{j}$ in the irregular trellis is 290 confined by the number of states $r_{j}$ in the corresponding trellis 291 structure, rather than by a constant number of states $r$, as in the 292 regular UEC trellis of [10]. In this way, the bit sequence $\mathbf{y}$ iden- 293 tifies a path through the single irregular trellis, which may be 294 represented by a vector $\mathbf{m}=\left[m_{j}\right]_{j=0}^{b}$ comprising $b+1$ state 295 values. As in the regular UEC trellis of [10], the transitions of 296 the proposed irregular trellis are synchronous with the unary 297 codewords of Table I. More specifically, just as each symbol 298 $x_{i}$ in the vector $\mathbf{x}$ corresponds to an $x_{i}$-bit codeword $\mathbf{y}_{i}$ in the 299 vector $\mathbf{y}$, the symbol $x_{i}$ also corresponds to a section $\mathbf{m}_{i}$ of 300 the trellis path $\mathbf{m}$ comprising $x_{i}$ transitions between $\left(x_{i}+1\right) \quad 301$ states. Owing to this, the path $\mathbf{m}$ is guaranteed to terminate 302 in the state $m_{b}=1$, when the symbol vector $\mathbf{x}$ has an even 303 length $a$, while $m_{b}=2$ is guaranteed when $a$ is odd [10]. Note 304 that the example unary-encoded bit sequence $\mathbf{y}=[1110010] 305$ corresponds to the path $\mathbf{m}=[1,3,5,3,2,1,1,2]$ through the 306 irregular UEC trellis of Fig. 2.

The path $\mathbf{m}$ may be modeled as a particular realization 308 of a vector $\mathbf{M}=\left[M_{j}\right]_{j=0}^{b}$ comprising $(b+1)$ RVs. Note that 309 the probability $\operatorname{Pr}\left(M_{j}=m_{j}, M_{j-1}=m_{j-1}\right)=P\left(m_{j}, m_{j-1}\right) 310$ of the transition from the previous state $m_{j-1}$ to the next state 311 $m_{j}$ can be derived by observing the value of each symbol in 312 the vector $\mathbf{x}$ and simultaneously its corresponding index. The 313 state transition $\mathbf{M}=\left\{M_{j}\right\}_{j=0}^{b}$ follows the same rule shown in 314 (4), and all the transitions can be categorised into four types, as 315 illustrated in $[10,(8)]$. Owing to this, the probability of a tran- 316 sition $P\left(m_{j}, m_{j-1}\right)$ in the irregular trellis is associated with the 317 transition probabilities $\operatorname{Pr}\left(M_{j}=m, M_{j-1}=m^{\prime}\right)=P\left(m, m^{\prime}\right) \quad 318$ in (5), shown at the bottom of the page. Note that these 319

$$
P\left(m_{j}, m_{j-1}\right)= \begin{cases}\frac{1}{2 l}\left[1-\sum_{x=1}^{\left\lceil\frac{m_{j-1}}{2}\right\rceil} P(x)\right] & \text { if } m_{j-1} \in\left\{1,2,3, \ldots, r_{j-1}-2\right\}, m_{j}=m_{j-1}+2 \\ \left.\frac{1}{2 l} P(x)\right|_{x=\left\lceil\frac{m_{j-1}}{2}\right\rceil} & \text { if } m_{j-1} \in\left\{1,2,3, \ldots, r_{j-1}-2\right\}, m_{j}=1+\text { odd }\left(m_{j-1}\right) \\ \frac{1}{2 l}\left[1-\sum_{x=1}^{\frac{r_{j-1}}{2}-1} P(x)\right] & \text { if } m_{j-1} \in\left\{r_{j-1}-1, r_{j-1}\right\}, m_{j}=1+\operatorname{odd}\left(m_{j-1}\right) \\ \frac{1}{2 l}\left[l-\frac{r_{j-1}}{2}-\sum_{x=1}^{\frac{r_{j-1}}{2}-1} P(x)\left(x-\frac{r_{j-1}}{2}\right)\right] & \text { if } m_{j-1} \in\left\{r_{j-1}-1, r_{j-1}\right\}, m_{j} \in\left\{r_{j}-1, r_{j}\right\} \\ 0 & \text { otherwise }\end{cases}
$$




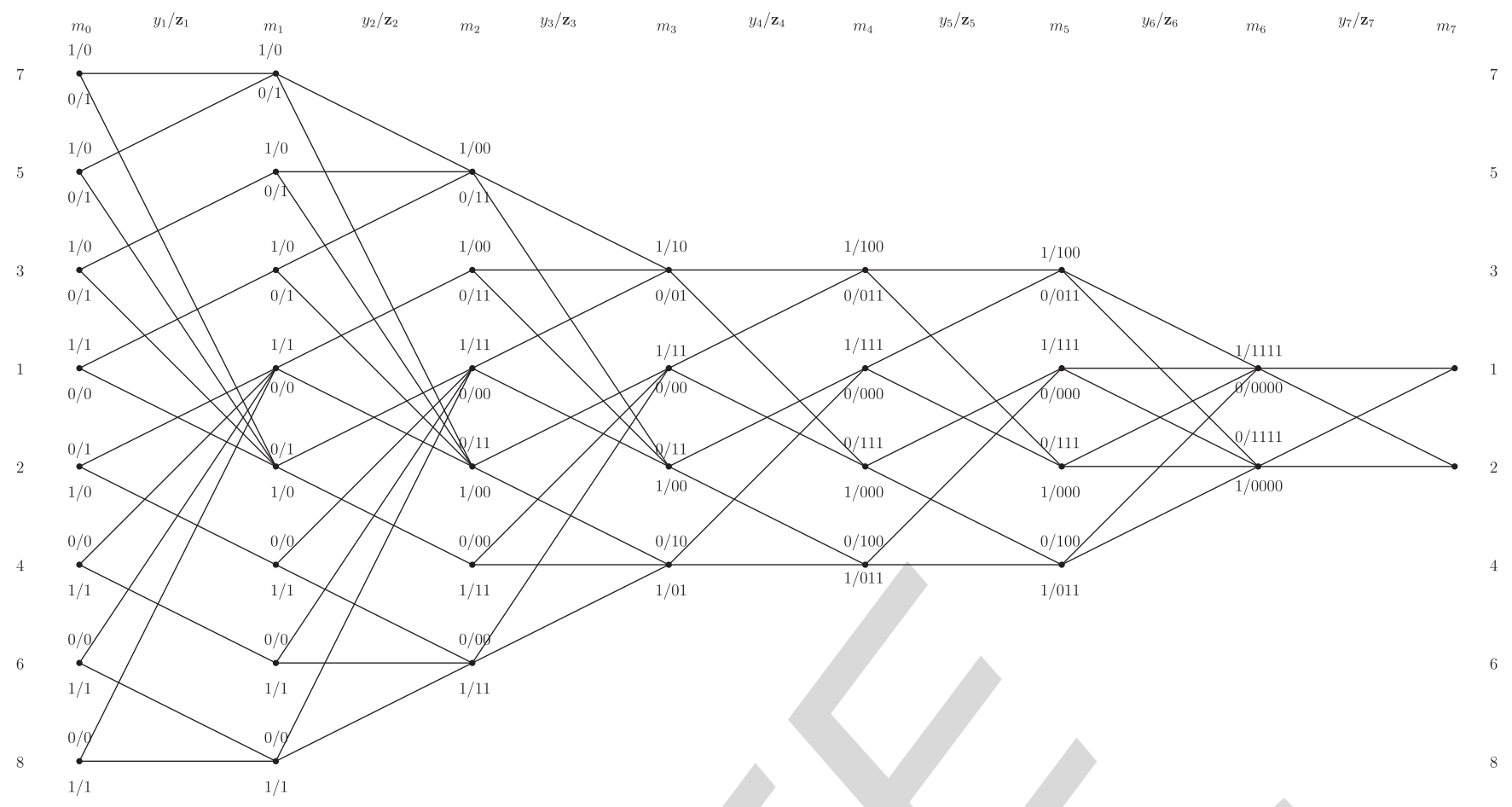

Fig. 2. An example of the proposed irregular UEC trellis, which is obtained by amalgamating seven different UEC trellises. Here, the component UEC codebooks $\mathbb{C}_{1}=\{0,1,1,1\}, \mathbb{C}_{2}=\{0,1,1,1\}, \mathbb{C}_{3}=\{000,000,000\}, \mathbb{C}_{4}=\{00,01\}, \mathbb{C}_{5}=\{000,011\}, \mathbb{C}_{6}=\{000,011\}$ and $\mathbb{C}_{7}=\{0000\}$ are employed.

transition probabilities are generalized, allow their application to any IrUEC trellis and to any source probability distribution $P(x)$.

Similar to the regular UEC trellis encoder, the proposed IrTrellis encoder represents each bit $y_{j}$ in the vector $\mathbf{y}$ by a codeword $\mathbf{z}_{j}$ comprising $n_{j}$ bits. This is selected from the corresponding set of $r_{j} / 2$ codewords $\mathbb{C}_{j}=\left\{\mathbf{c}_{1}^{j}, \mathbf{c}_{2}^{j}, \ldots, \mathbf{c}_{r_{j} / 2-1}^{j}\right.$, $\left.\mathbf{c}_{r_{j} / 2}^{j}\right\}$ or from the complementary set $\overline{\mathbb{C}}_{j}=\left\{\overline{\mathbf{c}_{1}^{j}}, \overline{\mathbf{c}_{2}^{j}}, \ldots\right.$, $\overline{\mathbf{c}_{r_{j} / 2-1}^{j}}, \overline{\mathbf{c}_{r_{j} / 2}^{j}}$, which is achieved according to

$$
\mathbf{z}_{j}=\left\{\begin{array}{ll}
\overline{\mathbf{c}_{\left\lceil m_{j-1} / 2\right\rceil}^{j}} & \text { if } y_{j}=\operatorname{odd}\left(m_{j-1}\right) \\
\mathbf{c}_{\left\lceil m_{j-1} / 2\right\rceil}^{j} & \text { if } y_{j} \neq \operatorname{odd}\left(m_{j-1}\right)
\end{array} .\right.
$$

Finally, the selected codewords are concatenated to obtain the bit vector $\mathbf{z}=\left[z_{k}\right]_{k=1}^{b \bar{n}}$ of Fig. 1 , where $\bar{n}=\frac{1}{b} \sum_{j=1}^{b} n_{j}$ is the average codeword length. For example, the path $\mathbf{m}=$ $[1,3,5,3,2,1,1,2]$ through the irregular UEC trellis of Fig. 2 yields the encoded bit sequence $\mathbf{z}=$ [1000011111110000], which comprises $b \bar{n}=16$ bits, where we have $\bar{n}=\frac{16}{7}$.

Note that the bit vector $\mathbf{z}$ may be modeled as a specific realization of a vector $\mathbf{Z}=\left[Z_{k}\right]_{k=1}^{b \bar{n}}$ comprising $b \bar{n}$ binary RVs. Observe in Fig. 2 that each of the $b$ component trellis structures in the irregular UEC trellis of the IrTrellis encoder is designed to obey symmetry and to rely on complementary codewords. Hence, bits of the encoded bit vector $\mathbf{Z}$ have equiprobable values, where $\operatorname{Pr}\left(Z_{k}=0\right)=\operatorname{Pr}\left(Z_{k}=1\right)=0.5$, and the bit entropy obeys $H_{Z_{k}}=H\left[\operatorname{Pr}\left(Z_{k}=0\right)\right]+H\left[\operatorname{Pr}\left(Z_{k}=\right.\right.$ $1)]=1$. Owing to this, in contrast to some of the benchmarkers to be considered in Section V, the proposed IrUEC scheme of Fig. 1 does not suffer from additional capacity loss.
We assume that each of the $b$ trellis structures in the proposed 346 irregular UEC trellis is selected from a set of $S$ component 347 UEC trellis structures $\left\{\mathrm{UEC}^{S}\right\}_{S=1}^{S}$, corresponding to a set of $S 348$ component codebooks $\left\{\mathbb{C}_{s}\right\}_{s=1}^{S}$. More specifically, we assume 349 that each codebook $\mathbb{C}_{S}$ is employed for generating a particu- 350 lar fraction $\alpha_{s}$ of the bits in $\mathbf{z}$, where we have $\sum_{s=1}^{S} \alpha_{s}=1.351$ Here, the number of bits generated using the codebook $\mathbb{C}_{s}$ is 352 given by $b \bar{n} \cdot \alpha_{s}$. We will in Section IV show that the fractions 353 $\alpha=\left\{\alpha_{S}\right\}_{s=1}^{S}$ may be designed in order to appropriately shape 354 the IrUEC EXIT function. Moreover, the IrUEC coding rate is 355 given by $R_{\mathrm{IrUEC}}=\sum_{s=1}^{S} \alpha_{s} \cdot R_{\mathrm{UEC}^{s}}$, where the corresponding 356 coding rate $R_{\mathrm{UEC}^{s}}$ of the regular $\mathrm{UEC}^{s}$ code depends on the 357 codebook $\mathbb{C}_{S}$ and is given by [10, Eq. (11)].

\section{IrURC Encoder and Modulator}

As shown in Fig. 1, the IrUEC-encoded bit sequence $\mathbf{z}$ is 360 interleaved in the block $\pi_{1}$ in order to obtain the bit vector $\mathbf{v}, 361$ which is encoded by an IrURC encoder [14], [20] comprising 362 $T$ component URC codes $\left\{\mathrm{URC}^{t}\right\}_{t=1}^{T}$. Unlike our IrUEC code, 363 each component URC code URC ${ }^{t}$ of the IrURC code employs 364 a separate trellis structure. This is necessary, since the final 365 state of each component URC code has no relation to the ini- 366 tial state of the subsequent component URC code, as described 367 in Section II-B. Therefore, the interleaved IrURC-encoded bit 368 vector $\mathbf{u}$ is decomposed into $T$ sub-vectors $\left\{\mathbf{u}_{t}\right\}_{t=1}^{T}$, each having 369 a length given by $b \bar{n} \cdot \beta_{t}$, where $\beta_{t}$ represents the specific frac- 370 tion of the bits in $\mathbf{v}$ that are encoded by the component $\mathrm{URC}^{t} 371$ code, which obeys $\sum_{t=1}^{T} \beta_{t}=1$. In Section IV, we also show 372 that the fractions $\beta=\left\{\beta_{t}\right\}_{t=1}^{T}$ may be designed in order to shape 373 the IrURC EXIT function. 
In common with each of its $T$ number of component URC codes, the IrURC code has a coding rate of $R_{\text {IrURC }}=1$, regardless of the particular irregular code design. Owing to this, each of the $T$ number of binary sub-vectors $\left\{\mathbf{v}_{t}\right\}_{t=1}^{T}$ that result from IrURC encoding has the same length as the corresponding subvector $\mathbf{u}_{t}$. The set of these sub-vectors $\left\{\mathbf{v}_{t}\right\}_{t=1}^{T}$ are concatenated to obtain the bit-vector $\mathbf{v}$, which comprises $b \bar{n}$ bits.

Finally, the IrURC-encoded bit vector $\mathbf{v}$ is interleaved by $\pi_{2}$ in order to obtain the bit vector $\mathbf{w}$, which is modulated onto the uncorrelated non-dispersive Rayleigh fading channel using Gray-mapped QPSK. The overall effective throughput of the proposed scheme is given by $\eta=R_{\mathrm{IrUEC}} \cdot R_{\mathrm{IrURC}} \cdot \log _{2}(M)$, where we have $M=4$ for QPSK.

\section{IRUEC-IRURC DECODER}

In this section, we introduce the receiver of the proposed IrUEC-IrURC scheme shown in Fig. 1. In analogy with the IrURC encoder, the IrURC decoder employs $T$ number of component URC decoders $\left\{\mathrm{URC}^{t}\right\}_{t=1}^{T}$, each having a distinct independent trellis structure. By contrast, the IrUEC employs a unary decoder and a novel IrTrellis decoder relying on a single irregular trellis. In Section III-A, the demodulator and the iterative operation of the IrURC and IrUEC decoders will be discussed, while in Sections III-B and III-C we will detail the internal operation of two components of the IrUEC decoder, namely of the IrTrellis decoder and of the unary decoder, respectively.

\section{A. Demodulator and Iterative Decoding}

As shown in Fig. 1, QPSK demodulation is employed by the receiver in order to obtain the vector $\tilde{\mathbf{w}}$ of Logarithmic Likelihood Ratios (LLRs), which pertain to the bits in the vector $\mathbf{w}$. This vector is deinterleaved by $\pi_{2}^{-1}$ for the sake of obtaining the LLR vector $\tilde{\mathbf{v}}$, which is decomposed into the $T$ sub-vectors $\left\{\tilde{\mathbf{v}}_{t}\right\}_{t=1}^{T}$ that have the same lengths as the corresponding sub-vectors of $\left\{\mathbf{v}_{t}\right\}_{t=1}^{T}$. Here, we assume that a small amount of side information is used for reliably conveying the lengths of all vectors in the IrUEC-IrURC transmitter to the receiver. The sub-vectors $\left\{\tilde{\mathbf{v}}_{t}\right\}_{t=1}^{T}$ are then input to the corresponding component URC decoders $\left\{\mathrm{URC}^{t}\right\}_{t=1}^{T}$ of the IrURC decoder.

Following this, iterative exchanges of the vectors of extrinsic LLRs [21] commences between the Soft-Input Soft-Output (SISO) IrUEC and IrURC decoders. In Fig. 1, the notation $\tilde{\mathbf{u}}$ and $\tilde{\mathbf{z}}$ represent vectors of LLRs pertaining to the bit vectors $\mathbf{u}$ and $\mathbf{z}$, which are related to the inner IrURC decoder and the outer IrUEC decoder, respectively. Additionally, a subscript of this notation denotes the dedicated role of the LLRs, with $a$, $e$ and $p$ indicating a priori, extrinsic and a posteriori LLRs, respectively.

At the beginning of iterative decoding, the a priori LLR vector $\tilde{\mathbf{u}}^{\mathrm{a}}$ is initialised with a vector of zeros, having the same length as the corresponding bit vector $\mathbf{u}$. As shown in the IrURC decoder of Fig. 1, the vector $\tilde{\mathbf{u}}^{\mathrm{a}}$ is decomposed into the $T$ sub-vectors $\left\{\tilde{\mathbf{u}}_{t}^{\mathrm{a}}\right\}_{t=1}^{T}$, which have the same lengths as the corresponding sub-vectors of $\left\{\mathbf{u}_{t}\right\}_{t=1}^{T}$. Together with $\left\{\tilde{\mathbf{v}}_{t}^{\mathrm{a}}\right\}_{t=1}^{T}$, the sub-vectors $\left\{\tilde{\mathbf{u}}_{t}^{\mathrm{a}}\right\}_{t=1}^{T}$ are fed to the corresponding URC decoder 429 $\mathrm{URC}^{t}$, which then outputs the resulting extrinsic LLR vectors 430 $\left\{\tilde{\mathbf{u}}_{t}^{\mathrm{e}}\right\}_{t=1}^{T}$ by employing the logarithmic Bahl-Cocke-Jelinek- 431 Raviv (BCJR) algorithm [22]. These vectors are combined for 432 forming the extrinsic LLR vector $\tilde{\mathbf{u}}^{\mathrm{e}}$ that pertains to the vec- 433 tor $\mathbf{u}$, which is sequentially deinterleaved by the block $\pi_{1}^{-1}$ in 434 order to obtain the a priori LLR vector $\tilde{\mathbf{z}}^{\mathrm{a}}$ that pertains to the 435 bit vector $\mathbf{z}$. Similarly, the IrTrellis decoder is provided with 436 the a priori LLR vector $\tilde{\mathbf{z}}^{\mathrm{a}}$ and generates the vector of extrinsic 437 LLRs $\tilde{\mathbf{z}}^{\mathrm{e}}$, which are interleaved in the block $\pi_{1}$ to obtain the $a 438$ priori LLR vector $\tilde{\mathbf{u}}^{\mathrm{a}}$ that is provided for the next iteration of 439 the IrURC decoder.

\section{B. IrTrellis Decoder}

As discussed in Section II, our IrUEC code employs a novel 442 bit-based irregular trellis, while the IrURC code employs a 443 selection of independent trellises. The novel IrTrellis decoder 444 within the IrUEC decoder applies the BCJR algorithm to the 445 irregular trellis. The synchronization between the novel irreg- 446 ular trellis and the unary codewords is exploited during the 447 BCJR algorithm's $\gamma_{t}$ calculation of [22, (9)]. This employs 448 the conditional transition probability $\operatorname{Pr}\left(M_{j}=m_{j} \mid M_{j-1}=449\right.$ $m_{j-1}$ ), where we have

$$
P\left(m_{j} \mid m_{j-1}\right)=\frac{P\left(m_{j}, m_{j-1}\right)}{\sum_{\check{m}=1}^{r_{j}} P\left(\check{m}, m_{j-1}\right)}
$$

and $P\left(m_{j}, m_{j-1}\right)$ is given in (5).

Note that the IrUEC decoder will have an EXIT function 452 [23] that reaches the $(1,1)$ point of perfect convergence to an 453 infinitesimally low Symbol Error Ratio (SER), provided that all 454 component codebooks in the set $\left\{\mathbb{C}_{S}\right\}_{S=1}^{S}$ have a free distance of 455 at least 2 [24], as characterised in Section IV. Since the combi- 456 nation of the IrURC decoder and demodulator will also have an 457 EXIT curve that reaches the $(1,1)$ point in the top right corner 458 of the EXIT chart, iterative decoding convergence towards the 459 Maximum Likelihood (ML) performance is facilitated [25]. At 460 this point, the IrTrellis decoder may invoke the BCJR algorithm 461 for generating the vector of a posteriori LLRs $\tilde{\mathbf{y}}^{\mathrm{p}}$ that pertain to 462 the corresponding bits in the vector $\mathbf{y}$.

\section{Unary Decoder}

As described in [10], the unary decoder of Fig. 1 sorts the 465 values in the LLR vector $\tilde{\mathbf{y}}^{\mathrm{p}}$ in order to identify the $a$ number of 466 bits in the vector $\mathbf{y}$ that are most likely to have values of zero. 467 A hard decision vector $\hat{\mathbf{y}}$ is then obtained by setting the value of 468 these bits to zero and the value of all other bits to one. Finally, 469 the bit vector $\hat{\mathbf{y}}$ can be unary decoded in order to obtain the 470 symbol vector $\hat{\mathbf{x}}$ of Fig. 1, which is guaranteed to comprise $a \quad 471$ number of symbols.

\section{Algorithm For the Parametrization of the} IRUEC-IRURC SCHEME

The performance of the IrUEC-IrURC scheme depends on 475 how well it is parametrized. A good parametrization is one that 476 


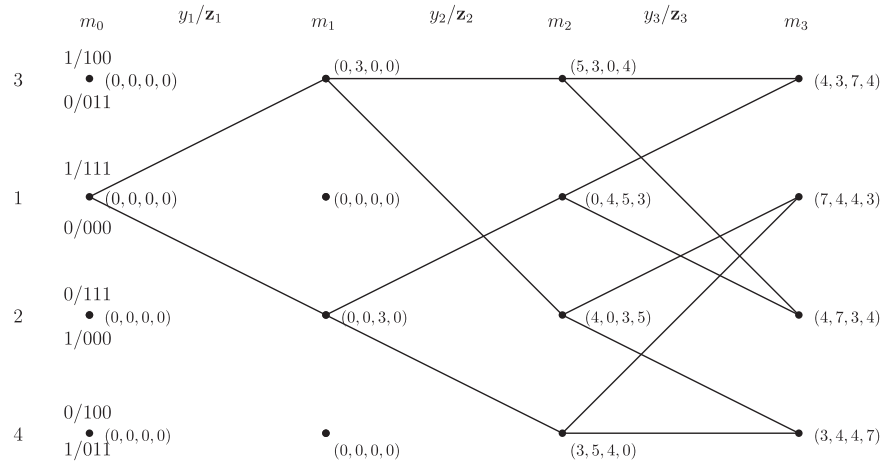

Fig. 3. The legitimate paths through the first three stages in UEC trellis having the codewords $\mathbb{C}=\{000,011\}$.

results in a narrow but still open EXIT chart tunnel, although achieving this requires a high degree of design freedom, when shaping the IrUEC and IrURC EXIT functions. Therefore, we begin in Section IV-A by characterising the free distance property of the UEC codes and selecting a set of UEC component codes having a wide variety of different inverted EXIT function shapes. This maximises the degree of freedom that is afforded, when matching the IrUEC EXIT function to that of the IrURC code. In Section IV-B, we propose a novel extension to the double-sided EXIT chart matching algorithm of [14], which we employ for jointly matching the EXIT functions of the IrUEC and the IrURC codes. However, in contrast to the algorithm of [14], which does not allow a particular coding rate to be targeted for the IrUEC-IrURC scheme, our algorithm designs both the fractions $\alpha$ and $\beta$ to achieve a particular target coding rate. In Section V, this will be exploited to facilitate a fair comparison with benchmarkers having particular coding rates.

\section{A. Design of UEC Component Codes}

Since an $r$-state $n$-bit UEC code is parametrized by a codebook set $\mathbb{C}$ comprising $r / 2$ number of codewords each having $n$ bits, there are a total of $2^{n \cdot r / 2}$ number of candidates for $\mathbb{C}$. It is neither possible nor necessary to employ all these $2^{n \cdot r / 2}$ codebooks as the component codes in our IrUEC code, because some of the codebooks will have identical or similar inverted EXIT function shapes, offering no additional degree of freedom, when performing EXIT chart matching. Therefore, it is desirable to eliminate these candidate codebooks.

The generalised UEC trellis structure associated with the codebook $\mathbb{C}=\left\{\mathbf{c}_{1}, \mathbf{c}_{2}, \ldots, \mathbf{c}_{r / 2-1}, \mathbf{c}_{r / 2}\right\}$ is depicted in [10, Fig. 3(a)]. Note that the upper half and the lower half of the trellis is symmetrical in terms of the output codewords $z_{j}$ generated in response to a given input bit value $y_{j}$, as shown in (6). More specifically, for the states in the upper half of the trellis, the output codewords $z_{j}$ are selected from the codebook $\mathbb{C}$ when $y_{j}=0$, while the codewords from its complementary codebook $\overline{\mathbb{C}}=\left\{\overline{\mathbf{c}_{1}}, \overline{\mathbf{c}_{2}}, \ldots, \overline{\mathbf{c}_{r / 2-1}}, \overline{\mathbf{c}_{r / 2}}\right\}$ are selected when $y_{j}=1$. For the states in the lower half of the trellis, the output codewords $z_{j}$ are selected from the codebook $\mathbb{C}$ when $y_{j}=1$ and from the complementary codebook $\overline{\mathbb{C}}$ when $y_{j}=0$. Intuitively, if any particular subset of the $n$ bits at the same positions within each codeword of $\mathbb{C}$ are inverted, this would not change the distance properties of the output bit vector $\mathbf{z}$, hence resulting in an identical inverted EXIT function. For example, inverting 519 the first bit of each codeword in the codebook $\mathbb{C}_{0}=\{00,01\} 520$ will give a new codebook $\mathbb{C}_{1}=\{10,11\}$ having an identical 521 EXIT function. Likewise, inverting both bits of the codewords 522 in $\mathbb{C}_{0}$ will give $\mathbb{C}_{2}=\{11,10\}$, which also has an identical EXIT 523 function. Similarly, swapping any pair of the $n$ bits at the same 524 positions between each pair of codewords will not affect the 525 distance properties or the shape of the inverted EXIT function 526 either. For example, swapping the two bits in the codebook $\mathbb{C}_{0} 527$ results in a new codebook $\mathbb{C}_{3}=\{00,10\}$, having an identical 528 inverted UEC EXIT function shape. Therefore, each of these 529 four codebooks, $\mathbb{C}_{0}, \mathbb{C}_{1}, \mathbb{C}_{2}$ and $\mathbb{C}_{3}$, as well as their conversions 530 created by bit-inversion and swapping, have identical inverted 531 EXIT functions. Consequently, all but one of these codebooks 532 can be eliminated as candidates for the sake of reducing the 533 complexity of EXIT chart matching.

The number of candidate UEC codebooks may be further 535 reduced by characterising their free distance properties. Since 536 no analytic method has been developed for calculating the free 537 distance $d_{f}$ of a UEC code, we propose a heuristic method 538 for obtaining an approximate measure of $d_{f}$. The free dis- 539 tance represents the minimum distance between any pair of 540 encoded bit vectors produced by different paths through the 541 trellis. The total number of possible pairings of paths emerg- 542 ing from a particular state in a UEC trellis of length $b$ is given 543 by $2^{b-1}\left(2^{b}-1\right)$, which grows exponentially. However, consid- 544 ering the symmetry of a regular UEC trellis, it is possible to 545 use a step-by-step directed search for determining the free dis- 546 tance, rather than using a brute force exhaustive search. Note 547 that in the regular UEC trellis as generalised in [10, Fig. 3(a)], 548 a bit vector $\mathbf{y}=\left[y_{j}\right]_{j=1}^{b}$ identifies a unique path $\mathbf{m}=\left[m_{j}\right]_{j=0}^{b} 549$ that emerges from state 1 and terminates at either state 1550 or 2, hence accordingly identifying a corresponding output 551 bit sequence $\mathbf{z}=\left[z_{k}\right]_{k=1}^{b \vec{n}}$. By exploiting this observation, the 552 free distance $d_{f}$ can be obtained by computing the Hamming 553 Distance(HD) between each pair of paths and then selecting the 554 pair having the minimum HD, whenever two paths merge at a 555 particular state in the trellis. 556

When the bit sequence length considered satisfies $b>557$ $r / 2$, the paths form complete trellis stages, as exemplified 558 in Fig. 3. Therefore, in order to reduce the search complex- 559 ity, we consider all permutations of the $b$-bit unary-encoded 560 vector $\mathbf{y}$ bit-by-bit, considering all paths that emerge from 561 state $m_{0}=1$ and terminate at each particular state $m_{b}=562$ $1,2, \ldots, r$, on a step-by-step basis. For a pair of states 563 $m_{j}, m_{j}^{\prime} \in\{1,2,3, \ldots, r\}$, we define $d_{m_{j}, m_{j}^{\prime}}^{j}$ as the minimum 564 HD between the set of all paths that terminate at state $m_{j} 565$ and the set that ends at state $m_{j}^{\prime}$, given the input bit sequence 566 $\left[y_{1}, y_{2}, \ldots, y_{j}\right]$, where $j \in\{0,1, \ldots, b\}$. Each state $m_{j}$ is 567 labelled as $\left(d_{m_{j}, 1}^{j}, d_{m_{j}, 2}^{j}, d_{m_{j}, 3}^{j}, \ldots, d_{m_{j}, r}^{j}\right)$, where we have 568 $d_{m_{j}, m_{j}^{\prime}}^{j}=d_{m_{j}^{\prime}, m_{j}}^{j}$. For each state $m_{0} \in\{1,2,3, \ldots, r\}$, the min- 569 imum HDs are initialized to 0s. Therefore, the distance $d_{m_{j}, m_{j}^{\prime}}^{j}$ can be calculated by

$$
d_{m_{j}, m_{j}^{\prime}}^{j}=\min _{m_{j-1}, m_{j-1}^{\prime}}\left[d_{m_{j-1}, m_{j-1}^{\prime}}^{j-1}+h\left(\mathbf{z}_{m_{j-1}, m_{j}}, \mathbf{z}_{m_{j-1}^{\prime}, m_{j}^{\prime}}\right)\right] .
$$


Here, $\mathbf{z}_{m_{j-1}, m_{j}}$ is the codeword in the set $\mathbb{C}$ or in the complementary set $\overline{\mathbb{C}}$ that is generated by the transition from state $m_{j-1}$ to state $m_{j}$, while the function $h(\cdot, \cdot)$ denotes the HD between the two operands. Owing to this, our method conceived for determining the free distance of a UEC code has a complexity order of $O[b \cdot r(r-1)]$, where $r$ is the number of states in the trellis and $b$ is the length of the bit vector $\mathbf{y}$ considered. Let $\mathbb{Y}_{b_{1}}$ be the bit sequence set associated with the set of all paths $\mathbb{M}_{b_{1}}$ having a length of $b_{1}$, while $\mathbb{Y}_{b_{2}}$ is the bit sequence set associated with the path set $\mathbb{M}_{b_{2}}$ having a length of $b_{2}$. Therefore, all sequences in $\mathbb{Y}_{b_{1}}$ are prefix of sequences in $\mathbb{Y}_{b_{2}}$, when we have $b_{1}<b_{2}$. For example, when $b_{1}=2$ and $b_{2}=3$, the bit sequence $\mathbf{y}_{2}=\{111011\}$ is a prefix of the bit sequence $\mathbf{y}_{3}=\{111011111\}$, where $\mathbf{y}_{2}$ is associated with the path vector $\mathbf{m}_{2}=\{1,3,2\}$ and $\mathbf{y}_{2}$ is associated with the path vector $\mathbf{m}_{2}=\{1,3,2,1\}$, respectively. Note that according to [26, Lemma 1$]$, the minimum $\mathrm{HD} d_{f}\left(\mathbb{Y}_{b_{1}}\right)$ among all bit sequences in $\mathbb{Y}_{b_{1}}$ is an upper bound on the minimum HD $d_{f}\left(\mathbb{Y}_{b_{2}}\right)$ of $\mathbb{Y}_{b_{2}}$, when we have $b_{1}<b_{2}$. Owing to this, the approximate free distance $d_{f}$ calculated using our method converges to the true free distance, as the lengths of the paths considered are extended towards infinity. In our experiments, we considered bit vector lengths of up to $b=10 r$. In all cases, we found that the free distance has converged before that point, regardless of how the UEC code is parametrised, owing to the common features of all UEC codes described in Section II-B."

For example, Fig. 3 shows all of the legitimate paths through an $r=4$-state trellis employing the codebook $\mathbb{C}=$ $\{000,011\}$ that may be caused by the first three bits in a bit vector $\mathbf{y}=\left\{y_{j}\right\}_{j=1}^{b}$, having a length $b>3$. Particularly, the minimum HD $d_{2,3}^{1}$ between states $m_{1}=2$ and $m_{1}^{\prime}=3$ is given by $d_{2,3}^{1}=d_{1,1}^{0}+h(111,000)=3$. Since there are no legitimate paths leading to the states $m_{1}=1$ or $m_{1}=$ 4 , we do not update the associated distances, as shown in Fig. 3. Similarly, we have $d_{1,2}^{2}=d_{2,3}^{1}+h(111,011)=4$, and $d_{1,2}^{3}=\min \left(d_{1,2}^{2}+h(000,111), d_{1,4}^{2}+h(000,100), d_{2,3}^{2}+\right.$ $\left.h(111,011), d_{3,4}^{2}+h(011,100)\right)=4$. Once the forward recursion has considered a sufficient number of trellis stages for $\min \left(d_{1,1}^{j}, d_{1,2}^{j}, d_{2,2}^{j}\right)=\min \left(d_{1,1}^{j-1}, d_{1,2}^{j-1}, d_{2,2}^{j-1}\right)$, then the approximate free distance becomes $d_{f}=\min \left(d_{1,1}^{j}, d_{1,2}^{j}, d_{2,2}^{j}\right)$.

Our set of candidate component UEC codes was further reduced by considering their free distances. More specifically, in order to achieve a wide variety of EXIT function shapes, we retained only UEC codebooks having the maximal or minimal free distances for each combination of $n \in\{2,3,4\}$ and $r \in\{2,4\}$, where a free distance of 3 is the minimal value that facilitates convergence to the $(1,1)$ point $[24]$ and avoids an error floor. We drew the EXIT functions for all remaining candidate component UEC codes and selected the five codebooks offering the largest variety of EXIT function shapes, as listed in Table II. Our experiments revealed that only insignificant EXIT function shape variations are obtained, when considering more than $r=4$ states. Without loss of generality, our irregular trellis example of Fig. 2 is constructed by concatenating the five UEC codebooks of Table II. In the following simulations, we will consider irregular trellises that are constructed using these
TABLE II

AFter InVERTING AND SWAPPING, We SELECT the IRUEC COMPONENT UEC CODEBOoKS $\left\{\mathbb{C}_{s}\right\}_{s=1}^{5}$ WITH $n$ BITS AND $r$ STATES Both up to 4 . All the Codebooks ARe in the Format $\left(\mathbb{C}_{s}, d_{f}\right)$, Where $d_{f}$ IS The Approximate Free Distance

\begin{tabular}{|l|l|l|}
\hline$n$ & $r=2$ & $r=4$ \\
\hline 2 & & $(\{00,01\}, 3)$ \\
\hline 3 & $(\{000\}, 3)$ & $(\{000,011\}, 4)$ \\
\hline 4 & $(\{0000\}, 4)$ & $(\{0000,0111\}, 5)$ \\
\hline
\end{tabular}

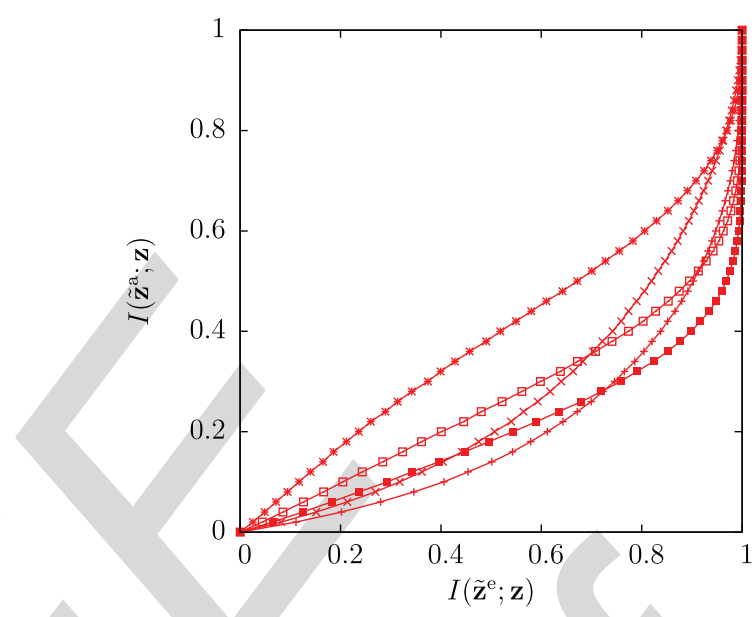

Fig. 4. Inverted EXIT functions for the $S=5$ component UEC codes $\left\{\mathrm{UEC}^{S}\right\}_{s=1}^{5}$ of Table II, when extended to $r=10$ states codebooks, and when the symbol values obey a zeta probability distribution having the parameter value $p_{1}=0.797$.

codebooks. However, the number of states $r$ employed by our 628 five UEC component codes can be optionally and independently 629 increased in the receiver, in order to facilitate nearer-to-capacity 630 operation at the cost of an increased decoding complexity [10]. 631 This is achieved by repeating the last element in the code- 632 book. For example, while the transmitter may use the codebook 633 $\mathbb{C}=\{00,01\}$, the receiver may extend this to the $r=10$-state 634 codebook $\mathbb{C}=\{00,01,01,01,01\}$. Fig. 4 plots the inverted 635 EXIT functions of the component UEC codes $\left\{\mathrm{UEC}^{s}\right\}_{s=1}^{5}, 636$ when extended to $r=10$ states. Note that, similar to the IrURC 637 EXIT function, the composite IrUEC EXIT function $f_{\text {IrUEC }}$ is 638 given as a weighted average of the component EXIT functions 639 $\left\{f_{\mathrm{UEC}^{s}}\right\}_{s=1}^{5}$, where we have

$$
f_{\mathrm{IrUEC}}=\sum_{s=1}^{5} \alpha_{s} \cdot f_{\mathrm{UEC}^{s}} .
$$

\section{B. Double-Sided EXIT Chart Matching Algorithm}

The sixth column of Table III provides the specific $E_{\mathrm{b}} / N_{0} 642$ values, where the DCMC capacity becomes equal to the 643 throughput $\eta$ of each scheme considered. These $E_{\mathrm{b}} / N_{0}$ values 644 represent the capacity bound, above which it is theoretically 645 possible to achieve reliable communication. Note that the 646 capacity bound is a function of the overall effective throughput 647 $\eta$ of the proposed IrUEC scheme, as described in Section II- 648 C. In turn, the overall effective throughput $\eta$ depends on the 649 
TABLE III

Characteristics of the VArious Schemes Considered, InCluding Outer Coding RATE $R_{\mathrm{O}}$, InNer Coding RATE $R_{\mathrm{I}}$ AND EFFECTIVE Throughput $\eta$. $E_{\mathrm{B}} / N_{0}$ Bounds ARE GIVEN For THE CASE OF GRAY-CODED QPSK TRANSMISSION OVER an Uncorrelated Narrowband Rayleigh Fading Channel. Complexity is QuANTIFIEd By the Average Number of ACS Operations InCURRed Per DeCOding Iteration ANd PER Bit in the Vector $\mathbf{z}$

\begin{tabular}{|c|c|c|c|c|c|c|c|c|}
\hline 1 & 2 & 3 & 4 & 5 & 6 & 7 & 8 & 9 \\
\hline Scheme & Codebooks & $R_{\mathrm{o}}$ & $R_{\mathrm{i}}$ & $\eta$ & $\begin{array}{c}E_{\mathrm{b}} / N_{0}[\mathrm{~dB}] \\
\text { capacity bound }\end{array}$ & $\begin{array}{c}E_{\mathrm{b}} / N_{0}[\mathrm{~dB}] \\
\text { area bound }\end{array}$ & $\begin{array}{l}E_{\mathrm{b}} / N_{0}[\mathrm{~dB}] \\
\text { tunnel bound }\end{array}$ & Complexity \\
\hline IrUEC-IrURC & $\multirow{3}{*}{\mathrm{UEC}^{s}}_{s=1}^{5}$ & \multirow{4}{*}{0.254} & \multirow{4}{*}{1} & \multirow{7}{*}{0.508} & \multirow{7}{*}{-0.05} & 0.21 & $\overline{0.3}$ & 258 \\
\hline IrUEC(med)-IrURC & & & & & & 0.30 & 0.6 & 192 \\
\hline IrUEC(low)-IrURC & & & & & & 1.14 & 1.2 & 157 \\
\hline UEC-IrURC & $\{000,011\}$ & & & & & 0.49 & 1.7 & 120 \\
\hline \multirow{2}{*}{ EG-IrCC-IrURC } & $\left\{\mathbf{C C}_{\mathrm{sys}}^{s}\right\}_{s=1}^{13}$ & 0.667 & 0.576 & & & 1.72 & 2.0 & 341 \\
\hline & $\left\{\mathbf{C C}_{\mathrm{ns}}^{\mathrm{s}}\right\}_{s=1}^{11}$ & \multirow{2}{*}{0.254} & \multirow[b]{2}{*}{1} & & & 1.02 & 1.1 & 146 \\
\hline EG-CC-IrURC & $([4,7,7], 6,6)$ & & & & & 1.00 & 2.2 & 132 \\
\hline
\end{tabular}

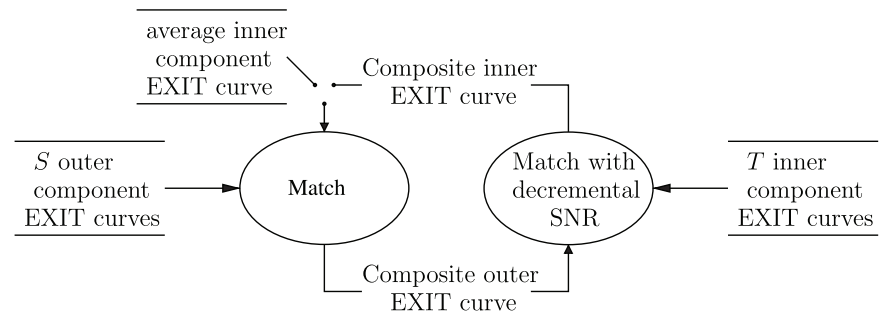

Fig. 5. Data-flow diagram of the proposed double-sided EXIT chart matching algorithm.

IrUEC coding rate $R_{\text {IrUEC }}$, which depends on the entropy of the zeta distribution $H_{X}$, as described in Section II-A. In order to facilitate the creation of an open EXIT chart tunnel, it is necessary, but not sufficient, for the area $A_{\mathrm{o}}$ beneath the inverted outer EXIT function to exceed the area $A_{\mathrm{i}}$ beneath the inner EXIT function [17]. Therefore, the area bound provides the $E_{\mathrm{b}} / N_{0}$ values where we have $A_{\mathrm{o}}=A_{\mathrm{i}}$, which would theoretically allow the creation of an open EXIT chart tunnel [27], if the outer and inner EXIT functions were shaped to match each other. Here, $A_{0}$ and $A_{\mathrm{i}}$ are the areas beneath the outer and inner EXIT functions, respectively. Depending on how well the EXIT functions match each other, a narrow but open EXIT chart tunnel can only be created at a specific $E_{\mathrm{b}} / N_{0}$ value, which we refer to as the tunnel bound. Based on these observations, the $E_{\mathrm{b}} / N_{0}$ difference between the capacity bound and the area bound quantifies the capacity loss that is mitigated by JSCC, while the difference between the area bound and the tunnel bound quantifies the capacity loss that is mitigated by irregular coding [28]. Based on this observation, our doublesided EXIT chart matching algorithm may be iteratively applied in order to match a pair of composite outer and inner EXIT functions, which are formed as a combination of $S$ component UEC EXIT functions and $T$ constituent URC EXIT functions, where the latter depend on the $E_{\mathrm{b}} / N_{0}$ value of the channel. In this way, a narrow but open EXIT chart tunnel between the inverted IrUEC EXIT function and the inner IrURC EXIT function may be created at $E_{\mathrm{b}} / N_{0}$ values that approach the capacity and area bounds, hence avoiding capacity loss and facilitating near-capacity operation.

As depicted in the data-flow diagram of Fig. 5, the algorithm commences by selecting the fractions $\alpha$, in order to yield an IrUEC code design having a particular coding rate $R_{\text {IrUEC }}$ and a composite IrUEC EXIT function that is shaped to match the 682 average of $T$ URC EXIT functions that correspond to a partic- 683 ular $E_{\mathrm{b}} / N_{0}$ value. The technique of [14] may be employed for 684 selecting the fractions $\beta$, in order to yield a composite IrURC 685 EXIT function that is shaped to match that of the IrUEC code. 686 Following this, the algorithm alternates between the matching 687 of the composite IrUEC EXIT function to the composite IrURC 688 EXIT function and vice versa, as shown in Fig. 5. In order 689 to facilitate near-capacity operation, we use a $0.1 \mathrm{~dB} E_{\mathrm{b}} / N_{0} 690$ decrement per iteration for the component URC EXIT func- 691 tions, when designing the fractions $\beta$ for the IrURC code, until 692 we find the lowest $E_{\mathrm{b}} / N_{0}$ value that achieves a marginally open 693 EXIT tunnel. Note that the double-sided EXIT chart matching 694 algorithm allows the design of an IrUEC code having a spe- 695 cific coding rate $R_{\text {IrUEC }}$. This enables us to design the IrUEC 696 code to have a coding rate of $R_{\mathrm{IrUEC}}=0.254$, which provides 697 a fair performance comparison with the regular UEC-IrURC 698 scheme of [10] and with other benchmarkers, as detailed in 699 Section V. More specifically, this results in the same overall 700 effective throughput of $\eta=R_{\mathrm{IrUEC}} \cdot R_{\mathrm{IrURC}} \cdot \log _{2}(M)=0.508701$ $\mathrm{bit} / \mathrm{s} / \mathrm{Hz}$, as listed in Table III.

For the IrURC encoder, we employ the $T=10$-component 703 URC codes $\left\{\mathrm{URC}^{t}\right\}_{t=1}^{10}$ of [20], [29]. After running the double- 704 sided EXIT chart matching algorithm of Fig. 5 until the $E_{\mathrm{b}} / N_{0} \quad 705$ value cannot be reduced any further without closing the EXIT 706 chart tunnel, the composite EXIT functions of the IrUEC and 707 IrURC schemes are obtained, as depicted in Fig. 6(a). Here, the 708 $E_{\mathrm{b}} / N_{0}$ value is $0.3 \mathrm{~dB}$, which is $0.35 \mathrm{~dB}$ away from the DCMC 709 capacity bound of $-0.05 \mathrm{~dB}$ and was found to be the lowest one 710 that creates an open EXIT chart tunnel. More specifically, the 711 fractions of the bit vector $\mathbf{z}$ that are generated by the constituent 712 UEC codes $\left\{\mathrm{UEC}^{s}\right\}_{s=1}^{5}$ of the IrUEC encoder are $\alpha=\left[\begin{array}{lll}0 & 0.7240 & 713\end{array}\right.$ $0.092400 .1836]$, respectively. Similarly, the fractions of the bit 714 vector $\mathbf{u}$ that encoded by the constituent URC codes $\left\{\mathrm{URC}^{t}\right\}_{t=1}^{10} 715$ of the IrURC encoder are $\beta=\left[\begin{array}{lllllllllll}0.1767 & 0 & 0.8233 & 0 & 0 & 0 & 0 & 0 & 0 & 0\end{array}\right], 716$ respectively.

\section{BENCHMARKERS AND SIMULATIONS}

In this section, we compare the SER performance of the 719 proposed IrUEC-IrURC scheme of Fig. 1 to that of various 720 SSCC and JSCC benchmarkers. As mentioned in Section IV, 721 the proposed IrUEC-IrURC scheme and all benchmarkers are 722 designed to have the same effective overall throughput of 723 


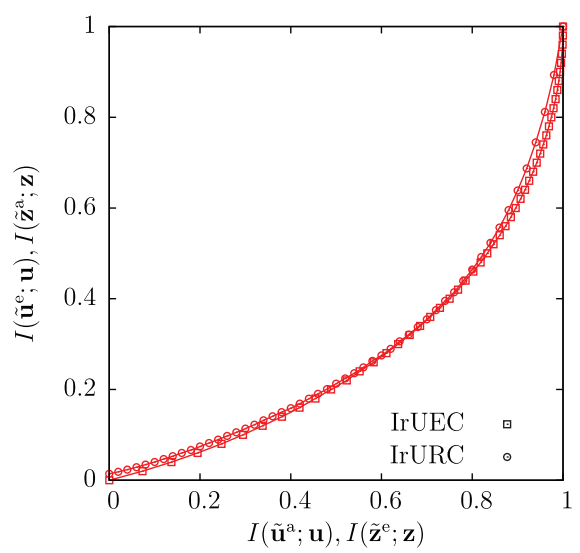

(a)

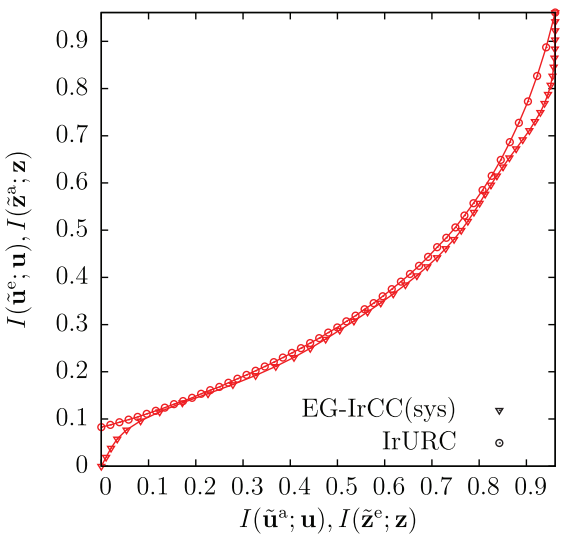

(b)

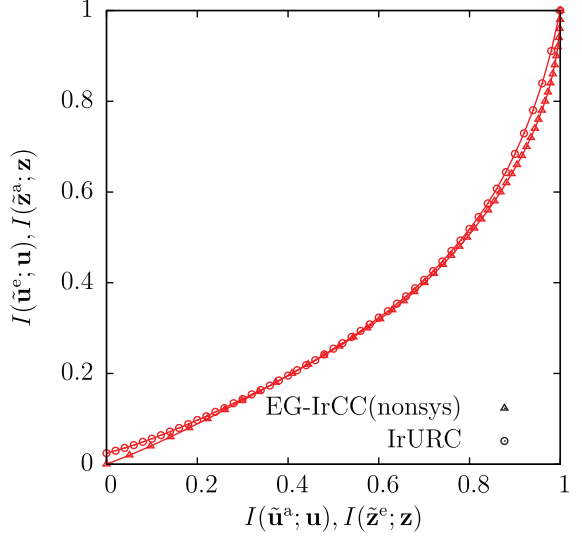

(c)

Fig. 6. Composite EXIT functions of (a) the IrUEC decoder employing $S=5$ component UEC codes $\left\{\text { UEC }^{s}\right\}_{s=1}^{5}$, (b) the EG-IrCC decoder employing the $S=13$ component recursive systematic CC codes $\left\{\mathbf{C C}_{\mathrm{sys}}^{s}\right\}_{s=1}^{13}$ and (c) the EG-IrCC scheme employing the $S=11$ component non-systematic CC codes $\left\{\mathbf{C C}_{\mathrm{ns}}^{s}\right\}_{s=1}^{11}$, and the IrURC scheme employing the $T=10$ component URC codes $\left\{\mathrm{URC}^{t}\right\}_{t=1}^{10}$, when conveying symbols obey a zeta distribution having the parameter $p_{1}=0.797$, and communicating over a QPSK-modulated uncorrelated narrowband Rayleigh fading channel. The EXIT chart tunnel is marginally open when $E_{\mathrm{b}} / N_{0}=0.3$, 2.0 and $1.1 \mathrm{~dB}$, respectively.
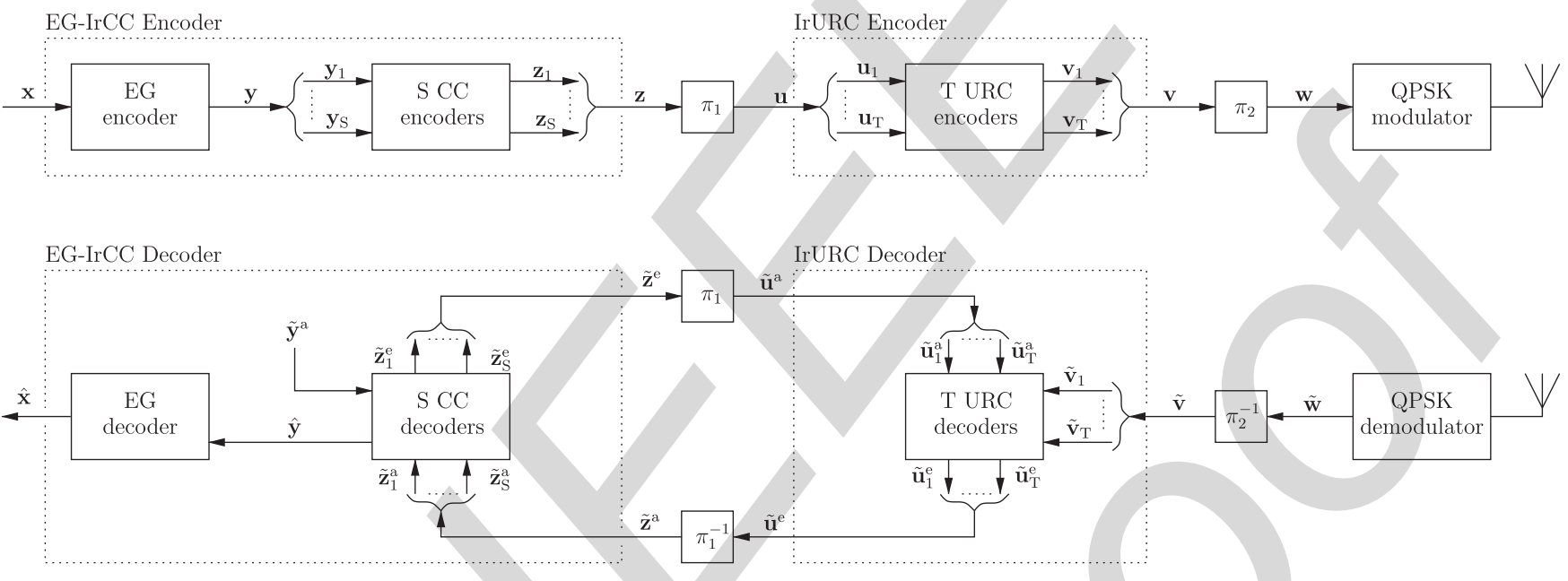

Fig. 7. Schematic of the EG-IrCC-IrURC benchmarker, in which an EG-IrCC code is serially concatenated with IrURC code and Gray-coded QPSK modulation schemes. Here, $\pi_{1}$ and $\pi_{2}$ represent interleavers, while $\pi_{1}^{-1}$ and $\pi_{2}^{-1}$ represent the corresponding deinterleavers.

$\eta=0.508 \mathrm{bit} / \mathrm{s} / \mathrm{Hz}$, for the sake of fair comparison. A pair of benchmarkers are constituted by the UEC-IrURC and EGCC-IrURC schemes of our previous work [10]. Furthermore, a new benchmarker is created by replacing the unary encoder and the IrTrellis encoder in the transmitter of Fig. 1 with an EG encoder and an IrCC encoder, respectively. This results in the SSCC benchmarker of Fig. 7, which we refer to as the EGIrCC-IrURC scheme. Table I shows the first ten codewords of the EG code, which are used for encoding the symbol vector $\mathbf{x}$.

As in the IrUEC-IrURC scheme, the bit vector $\mathbf{y}$ output by the EG encoder may be modeled as a realization of vector $\mathbf{Y}=$ $\left[Y_{j}\right]_{j=1}^{b}$ having binary RVs. However, as observed in [10], these RVs do not adopt equiprobable values $\operatorname{Pr}\left(Y_{j}=0\right) \neq \operatorname{Pr}\left(Y_{j}=\right.$ $1)$, hence giving a less than unity value for the corresponding bit entropy $H_{Y_{j}}$. Similarly, the bit vector $\mathbf{z}$ of Fig. 7 may be modeled as a particular realization of a vector $\mathbf{Z}=\left[Z_{k}\right]_{k=1}^{b \bar{n}}$ comprising $b \bar{n}$ binary RVs. Each binary RV $Z_{k}$ adopts the values 0 and 1 with the probabilities $\operatorname{Pr}\left(Z_{k}=0\right)$ and $\operatorname{Pr}\left(Z_{k}=1\right)$ respectively, corresponding to a bit entropy of $H_{Z_{k}}$. In the case where the IrCC code employs systematic component codes, the 743 bits of $\mathbf{y}$ having the entropy $H_{Y_{j}}<1$ will appear in $\mathbf{z}$, resulting 744 in a bit entropy of $H_{Z_{k}}<1$. However, a bit entropy of $H_{Z_{k}}<1745$ is associated with a capacity loss, as described in [10].

Hence, for the sake of avoiding any capacity loss, it is 747 necessary to use non-systematic recursive component codes, so 748 that the bits in the resultant encoded vector $\mathbf{z}$ have equiprob- 749 able values [10]. In order to demonstrate this, we introduce 750 two versions of the EG-IrCC-IrURC benchmarker. Firstly, 751 the $N=13$ recursive systematic component CC codes [15] 752 $\left\{\mathbf{C C}_{\text {sys }}^{s}\right\}_{s=1}^{13}$ that were originally proposed for IrCC encoding 753 are adopted in the EG-IrCC-IrURC encoder, as it will be 754 described in Section V-A. Secondly, Section V-B employs the 755 $S=11$ non-systematic recursive CC codebooks $\left\{\mathbf{C C}_{\mathrm{ns}}^{S}\right\}_{s=1}^{11} 756$ proposed in [20], in order to offer an improved version of the 757 EG-IrCC benchmarker. Meanwhile, the 10 component URC 758 codebooks $\left\{\mathrm{URC}^{t}\right\}_{t=1}^{10}$ employed by the IrURC encoder in both 759 versions of the benchmarker of Fig. 7 are identical to those in 760 the IrURC encoder of Fig. 1. 


\section{A. Recursive Systematic Component CC Codes}

763

764

765

766

767

768

769

770

771

772

773

774

775

776
The recursive systematic CC codes $\left\{\mathbf{C C}_{\text {sys }}^{s}\right\}_{s=1}^{13}$ employed in [15] were designed to have coding rates of $R_{\mathrm{CC}_{\mathrm{sys}}^{s}} \in$ $\{0.1,0.15, \ldots, 0.65,0.7\}$. However, since the EG-encoded bits in the vector $\mathbf{y}$ are not equiprobable, none of the systematic bits in the bit vector $\mathbf{z}$ will be equiprobable either. As a result, the coding rate $R_{\mathrm{CC}_{\mathrm{sys}}^{s}}=\frac{H_{Y_{j}}}{n_{\mathrm{CC}_{\mathrm{sys}}^{s}} \cdot H_{Z_{k}}^{\mathrm{CC}_{\mathrm{sys}}}}$ of each systematic $\mathrm{CC}$ will be lower than the above-mentioned values. Since each $\mathrm{CC}$ code $\mathbf{C C}_{\text {sys }}^{s}$ produces a different number of systematic bits, each will have a different bit entropy $H_{Z_{k}}^{\mathrm{CC}_{\text {sys }}^{s}}$, and the EXIT function of each CC code will converge to a different point $\left(H_{Z_{k}}^{\mathbf{C C}} \mathbf{C}_{\text {sys }}^{s}, H_{Z_{k}}^{\mathbf{C C}} \mathbf{C}_{\text {sys }}^{s}\right)$ in the EXIT chart [30]. The composite IrCC EXIT function will converge to a point $\left(H_{Z_{k}}^{\mathrm{IrCC}}, H_{Z_{k}}^{\mathrm{IrCC}}\right)$, where $H_{Z_{k}}^{\mathrm{IrCC}}$ is given by a weighted average of $\left\{H_{Z_{k}}^{\mathbf{C C}_{\text {sys }}^{s}}\right\}_{s=1}^{13}$, according to

$$
H_{Z_{k}}^{\mathrm{IrCC}}=\sum_{s=1}^{13} \alpha_{s} \cdot H_{Z_{k}}^{\mathbf{C C}_{\mathrm{sys}}^{s}} .
$$

Since the vector $\mathbf{z}$ is interleaved to generate the bit vector $\mathbf{u}$ as the input of the IrURC encoder, the IrURC EXIT function will also converge to $\left(H_{Z_{k}}^{\mathrm{IrCC}}, H_{Z_{k}}^{\mathrm{IrCC}}\right)$. However, this presents a particular challenge, when parametrizing the fractions $\alpha$ and $\beta$ of the EG-IrCC(sys)-IrURC scheme. More specifically, the fractions $\alpha$ vary as our double-sided EXIT chart matching algorithm progresses, causing the entropy $H_{Z_{k}}^{\mathrm{IrCC}}$ to vary as well. This in turn causes the IrURC EXIT function to vary, creating a cyclical dependency that cannot be readily resolved. More specifically, the fractions $\alpha$ must be selected to shape the EG-IrCC EXIT function so that it matches the IrURC EXIT function, but the IrURC EXIT function depends on the fractions $\alpha$ selected for the EG-IrCC EXIT function.

Owing to this, we design the fractions $\alpha$ and $\beta$ by assuming that the bits of $\mathbf{y}$ are equiprobable and by plotting the inverted EXIT functions for the $S=13$ recursive systematic CC codes accordingly, giving convergence to the $(1,1)$ point in Fig. 6(b). Then we invoke our double-sided EXIT matching algorithm to design the fractions $\alpha$ and $\beta$ for the $\operatorname{IrCC}$ (sys) and IrURC codes, which we apply to the EG-IrCC(sys)-IrURC scheme. For the case where the bits of the vector $\mathbf{y}$ have the non-equiprobable values that result from EG encoding, the composite EXIT functions are shown in Fig. 6(b). Here, the effective throughput is $\eta=0.508 \mathrm{bit} / \mathrm{s} / \mathrm{Hz}$ and the $E_{\mathrm{b}} / N_{0}$ value is $2.0 \mathrm{~dB}$, which is the lowest value for which an open EXIT chart tunnel can be created. This $E_{\mathrm{b}} / N_{0}$ tunnel bound is $2.05 \mathrm{~dB}$ away from the DCMC capacity bound of $-0.05 \mathrm{~dB}$, owing to the above-mentioned capacity loss. Furthermore, the EG-IrCC(sys)-IrURC scheme has an area bound of $1.72 \mathrm{~dB}$, which corresponds to a capacity loss of $1.77 \mathrm{~dB}$, relative to the capacity bound. The designed fractions for the EGIrCC scheme are $\alpha=\left[\begin{array}{ll}0.06200 .29970 .04970 .00040 .19430 \\ 0.098\end{array}\right.$ $0.09840 .128500000 .00020 .1668]$, while the fractions for the IrURC code are $\beta=\left[\begin{array}{lllllllllll}0.6548 & 0 & 0.3452 & 0 & 0 & 0 & 0 & 0 & 0 & 0\end{array}\right]$, respectively.

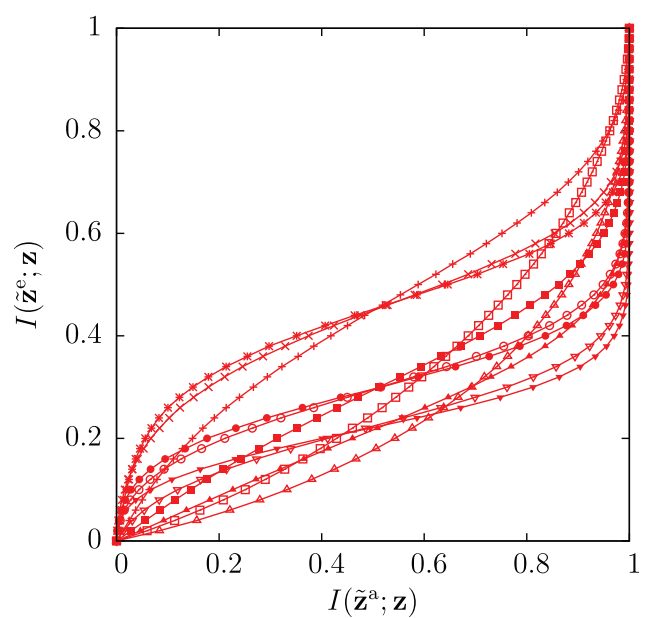

Fig. 8. Inverted EXIT functions for EG-CC code, for the case where the $S=11$ component recursive non-systematic CC codes $\left\{\mathbf{C C}_{\mathrm{ns}}^{S}\right\}_{s=1}^{11}$ are employed, and the symbol values obey a zeta probability distribution having the parameter value $p_{1}=0.797$.

\section{B. Recursive Non-Systematic Component CC Codes}

In order to avoid the capacity loss introduced by the recursive systematic $\mathrm{CC}$ codes, we advocate the recursive non-systematic $\mathrm{CC}$ codebooks $\left\{\mathbf{C C}_{\mathrm{ns}}^{s}\right\}_{s=1}^{11}$, which are described by the generator and feedback polynomials provided in [10, Table II]. More specifically, of the 12 codes presented in [10, Table II], we use all but the $r=2, n=2$ code, for the sake of avoiding an error floor. These recursive non-systematic CC codes attain the optimal distance properties [31] subject to the constraint of producing equiprobable bits $\operatorname{Pr}\left(Z_{j}=0\right)=\operatorname{Pr}\left(Z_{j}=1\right)$, which is necessary for avoiding any capacity loss. The inverted EXIT functions are plotted in Fig. 8.

For the sake of a fair comparison, we apply the doublesided EXIT chart matching algorithm of Fig. 5 again to design the EG-IrCC(nonsys)-IrURC scheme having a coding rate of $R_{\mathrm{EG}-\mathrm{IrCC}}=0.254$ and an effective throughput of $\eta=$ $0.508 \mathrm{bit} / \mathrm{s} / \mathrm{Hz}$. The composite EXIT functions of the EGIrCC(nonsys) and IrURC schemes are shown in Fig. 6(c). Here, the fractions of the EG-IrCC scheme are $\alpha=[0.810100 .0643$ 00000.1256000 ], while the fractions of the IrURC code are

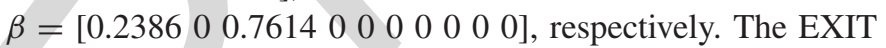
chart of Fig. 8 is provided for an $E_{\mathrm{b}} / N_{0}$ value of $1.1 \mathrm{~dB}$, which is the lowest value for which an open EXIT chart tunnel is created. As shown in Table III, this $E_{\mathrm{b}} / N_{0}$ tunnel bound is just $1.15 \mathrm{~dB}$ away from the DCMC capacity bound of $-0.05 \mathrm{~dB}$. This improvement relative to the EG-IrCC(sys)-IrURC scheme may be attributed to the non-systematic nature of the EGIrCC(nonsys)-IrURC scheme, which has reduced the capacity loss to $1.07 \mathrm{~dB}$, as quantified by considering the difference between the $E_{\mathrm{b}} / N_{0}$ area bound of $1.02 \mathrm{~dB}$ and the capacity bound.

\section{Parallel Component UEC Codes}

In order to make a comprehensive comparison, we also con- 844 sider a Parallel IrUEC-IrURC scheme. As shown in Fig. 9, 845 this scheme employs a parallel concatenation of $S$ number 846 

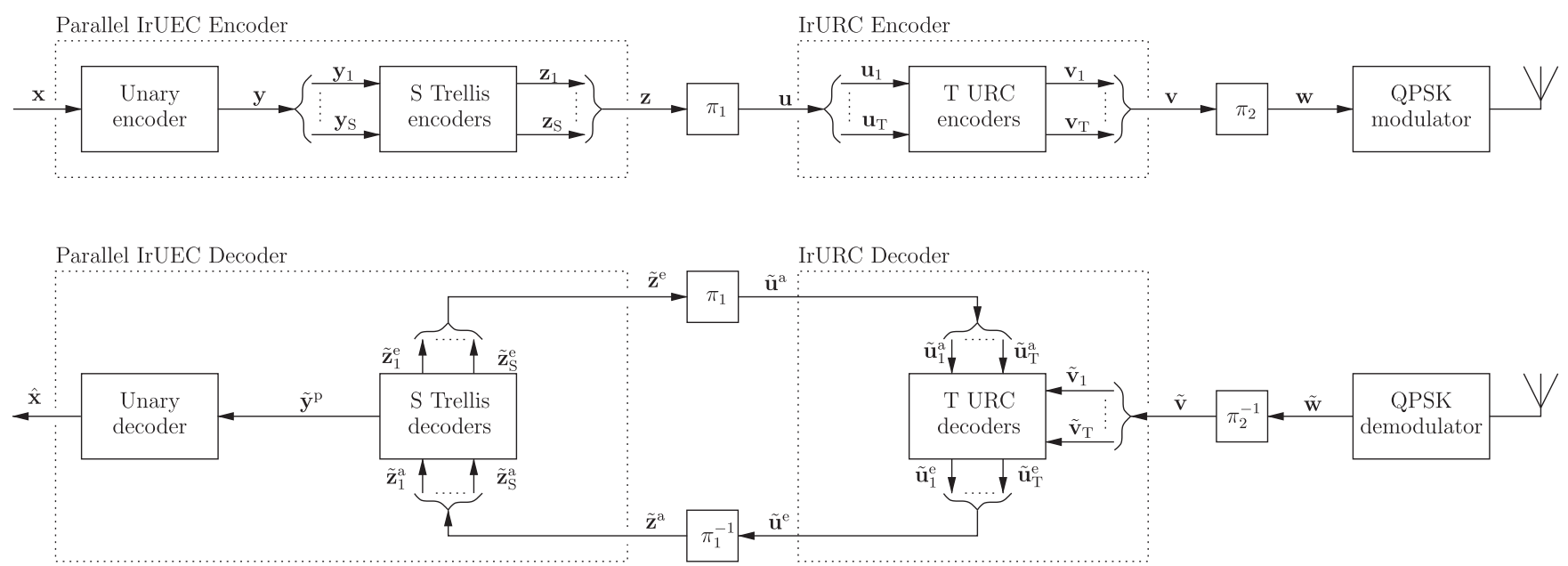

Fig. 9. Schematic of the Parallel IrUEC-IrURC benchmarker, in which a parallel IrUEC code is serially concatenated with IrURC code and Gray-coded QPSK modulation schemes. Here, $\pi_{1}$ and $\pi_{2}$ represent interleavers, while $\pi_{1}^{-1}$ and $\pi_{2}^{-1}$ represent the corresponding deinterleavers.

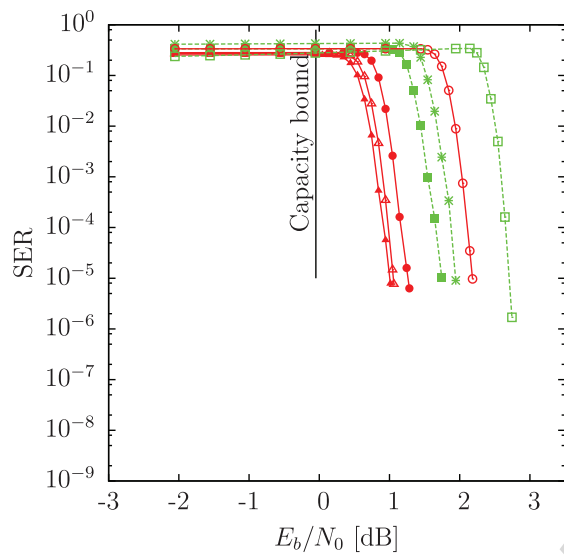

(a)

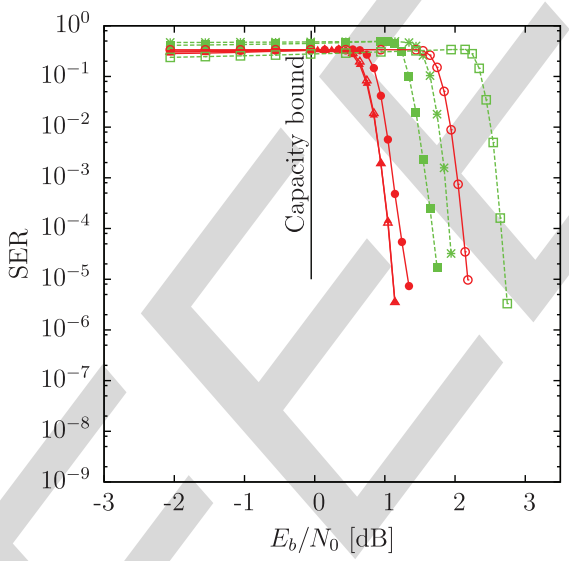

(b)

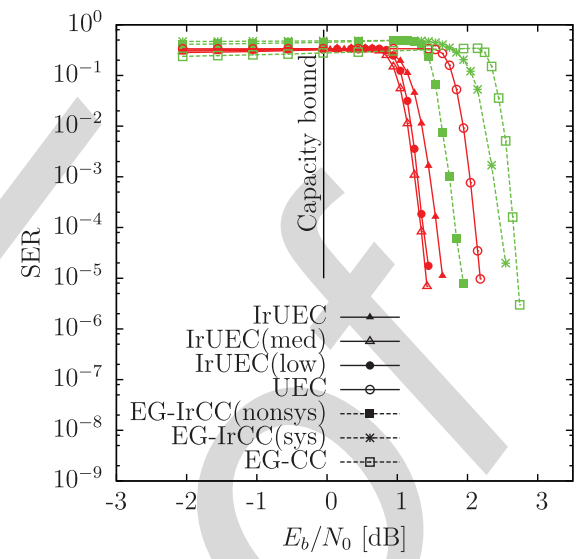

(c)

Fig. 10. SER performance for various arrangements of the proposed IrUEC-IrURC scheme of Fig. 1, the EG-IrCC-IrURC of Fig. 7, the Parallel IrUEC-IrURC scheme of Fig. 9, as well as the UEC-IrURC and the EG-IrURC schemes of [10], when conveying symbols obey a zeta distribution having the parameter $p_{1}=0.797$, and communicating over a QPSK-modulated uncorrelated narrowband Rayleigh fading channel having a range of $E_{\mathrm{b}} / N_{0}$ values. A complexity limit of (a) unlimited, (b) 10,000 and (c) 5,000 ACS operations per decoding iteration is imposed for decoding each of the bits in $\mathbf{z}$.

of separate UEC trellis encoders to encode the bit vector $\mathbf{y}$, in analogy with the structure of the EG-IrCC scheme. More specifically, the component UEC codes of the Parallel IrUEC encoder are selected from the five constituent codes provided in Table II, while the component UEC codes of the Parallel IrUEC decoder are extended to $r=10$ states. The irregular fractions employed by the Parallel IrUEC scheme are the same as those used in our proposed IrUEC scheme. However, in order for each component UEC trellis encoder to remain synchronized with the unary codewords in the bit vector $\mathbf{y}$, it is necessary for each component trellis to commence its encoding action from state $m_{0}=1$ and end at state $m_{b}=1$ or $m_{b}=2$. Owing to this, the subvectors of $\mathbf{y}$ input to each component UEC must comprise an integer number of complete unary codewords. The irregular coding fractions can only be controlled at the symbol level in the case of the parallel IrUEC scheme, rather than at the bit level, as in the proposed IrUEC scheme. Therefore, the corresponding EXIT chart of the parallel IrUEC scheme is not guaranteed to have an open tunnel, when the $E_{\mathrm{b}} / N_{0}$ value approaches the tunnel bound of Table III, hence resulting in a 866 degraded SER performance. However, if the frame length $a$ was 867 orders of magnitude higher, the difference between the symbol- 868 based and bit-based segmentations of the bit vector $\mathbf{y}$ would 869 become insignificantly small. As a result, a similar SER per- 870 formance may be expected for the parallel IrUEC scheme in 871 this case. In the following section, we will compare the perfor- 872 mances of the Parallel IrUEC and the proposed IrUEC schemes, 873 using different values for the frame length $a$.

\section{SER Results}

The SER performance of the IrUEC-IrURC, the EG- 876 IrCC(sys)-IrURC and the EG-IrCC(nonsys)-IrURC, UEC- 877 IrURC and EG-CC-IrURC schemes is characterised in Fig. 10. 878 In each case, the source symbol sequence $\mathbf{x}$ comprises $a=10^{4} 879$ symbols, the values of which obey a zeta distribution hav- 880 ing a parameter value of $p_{1}=0.797$. As shown above, the 881 parametrizations of the irregular codes in each scheme are 882 
designed to achieve the closest possible matching of EXIT charts, while giving the same overall effective throughput of $\eta=0.508 \mathrm{bit} / \mathrm{s} / \mathrm{Hz}$. Transmission is performed over a Graycoded QPSK-modulated uncorrelated narrowband Rayleigh fading channel, resulting in the DCMC capacity bound of $-0.05 \mathrm{~dB}$. We select two parametrizations of the schemes in [10] to create two of our four benchmarkers, namely the $r=4$-state UEC-IrURC and the $r=4$-state EG-CC-IrURC schemes. Note that the $r=4$-state EG-CC-IrURC scheme was found to outperform other parametrizations of the same scheme having higher number of states, owing to its superior EXIT chart matching accordingly. With the same effective throughput $\eta$, a fair comparison is provided between our proposed IrUEC-IrURC scheme and the four benchmarkers.

Note that the practical implementation of the time-variant IrTrellis used in our IrUEC-IrURC scheme follows the same principles as the parallel time-invariant trellises of the benchmarker schemes, such as the EG-IrCC-IrURC scheme and the regular UEC-IrURC scheme. Once the irregular coding fractions have been determined, the specific portions of message that should be encoded and decoded by the corresponding trellises are also determined. In both time-variant and parallel time-invariant trellises, the hardware is required to support different trellis structures, which may be implemented by appropriately changing the connections among the states of a single hardware implementation of a trellis. Although the proposed time-invariant trellis has some peculiarities at the interface between its different sections, these can also be implemented using the same hardware at either side of the interface. As an example platform for hardware implementation, the computation unit of [32] performs one ACS arithmetic operation per clock cycle, which are the fundamental operations used in BCJR decoders [18]. Therefore, the implementational complexity depends only on the computational complexity, as quantified per decoding iteration in Table III. Since a common computational complexity limit is used in our comparisons of the various schemes, they can be deemed to have the same implementational complexity. Although the routing and control of the proposed IrTrellis may be expected to be more complicated than in the parallel time-invariant trellises of the benchmarkers, it may be expected that the associated overhead is negligible compared to the overall implementational complexity.

As shown in Table III, our IrUEC-IrURC scheme imposes a complexity of 258 ACS operations per iteration per bit, when employing $r=10$ states for each component UEC code in the IrTrellis decoder. We also consider alternative parametrizations of our IrUEC-IrURC scheme, which employ an IrTrellis having fewer states, in order to achieve lower complexities. The IrUEC(med)-IrURC scheme relies on $r=6$ trellis states for different stages of the IrTrellis, which results in a total complexity of 192 ACS operations per iteration per bit. This matches that of the UEC-IrURC benchmarker. At the same time, the IrUEC(low)-IrURC scheme employs the minimal number of states for each stage of the IrTrellis, namely either $r=4$ states, as listed in Table II, hence resulting in a complexity of 157 ACS operations per iteration per bit.

During the simulation of each scheme, we recorded both the SER and the complexity incurred after each decoding iteration, resulting in a 3D plot of SER versus $E_{\mathrm{b}} / N_{0}$ and ver- 941 sus complexity. Fig. 10 presents 2D plots of SER versus $E_{\mathrm{b}} / N_{0} 942$ relationship, which were obtained by slicing through these 3D 943 plots at a particular complexity. More specifically, we select the 944 complexity limits of 10,000 and 5,000 ACS operations per iter- 945 ation per bit in Fig. 10(b) and (c), respectively. Meanwhile, 946 Fig.10 (a) characterizes the SER performance achieved after 947 iterative decoding convergence, regardless of the complexity. $\quad 948$

As shown in Table III, the proposed IrUEC-IrURC scheme 949 has an area bound of $0.21 \mathrm{~dB}$, which is the $E_{\mathrm{b}} / N_{0}$ value where 950 the area $A_{\mathrm{o}}$ beneath the inverted IrUEC EXIT function equals 951 that beneath the IrURC EXIT function. Although the UEC- 952 IrURC benchmarker has a similar area bound of $E_{\mathrm{b}} / N_{0}=953$ $0.49 \mathrm{~dB}$, it has an inferior EXIT chart matching capability 954 owing to its employment of regular UEC constituent codes. By 955 contrast, the employment of two irregular codes in the proposed 956 IrUEC-IrURC scheme facilitates an open EXIT chart tunnel at 957 an $E_{\mathrm{b}} / N_{0}$ value of $0.3 \mathrm{~dB}$, which is $1.4 \mathrm{~dB}$ lower than the open 958 tunnel bound of the UEC-IrURC benchmarker. Note that the 959 area and tunnel bounds are degraded in the context of the lower 960 complexity versions of the proposed IrUEC-IrURC scheme, 961 which have fewer states in the IrTrellis. This may be explained 962 by the increased capacity loss encountered when the number 963 of UEC states is reduced [10]. Note however that even with a 964 reduced complexity, the proposed IrUEC-IrURC scheme tends 965 to exhibit superior area and tunnel bounds, when compared 966 to the EG-IrCC-IrURC and EG-CC-IrURC benchmarkers, as 967 shown in Table III. This may be attributed to the large capacity 968 loss that is associated with SSCC scheme [10]. 969

Fig. 10 demonstrates that our proposed IrUEC-IrURC 970 scheme has a superior SER performance compared to all other 971 benchmarkers, regardless of which complexity limit is selected 972 in this particular scenario. For example, as shown in Fig. 10(a), 973 our IrUEC-IrURC scheme facilitates operation within $0.4 \mathrm{~dB}$ of 974 the capacity bound, offering a $0.8 \mathrm{~dB}$ gain compared to the EG- 975 IrCC(nonsys)-IrURC scheme, which is the best-performing of 976 the SSCC benchmarkers. This is achieved without any increase 977 in transmission energy, bandwidth, transmit duration or decod- 978 ing complexity. Note that the EG-IrCC(nonsys)-IrURC bench- 979 marker offers a $0.9 \mathrm{~dB}$ gain over the EG-IrCC(sys)-IrURC 980 benchmarker, which is owing to the capacity loss that is asso- 981 ciated with systematic IrCC component codes. As expected, 982 the reduced complexity versions of the proposed IrUEC-IrURC 983 scheme exhibit a degraded SER performance. However, the 984 IrUEC(low)-IrURC scheme can be seen to offer up to $0.5 \mathrm{~dB} 985$ gain over the UEC-IrURC benchmarker, which has a close 986 decoding complexity per bit per iteration. Since the Parallel 987 IrUEC-IrURC scheme can only provide a symbol-level con- 988 trol of the irregular coding fractions, the EXIT chart tunnel is 989 not guaranteed to be open at low $E_{\mathrm{b}} / N_{0}$ values. As a result, 990 Fig. 11 shows that the Parallel IrUEC-IrURC scheme of Fig. 9991 performs relatively poorly compared to the proposed IrUEC- 992 IrURC scheme, particularly when the frame length has values 993 of $a=10^{2}$ and $a=10^{3}$ symbols. Note that this performance 994 gain offered by the proposed scheme is obtained without impos- 995 ing any additional decoding complexity and without requiring 996 any additional transmission-energy, -bandwidth, or -duration. 997 In analogy with Fig. 10(a), an additional set of SER results 998 


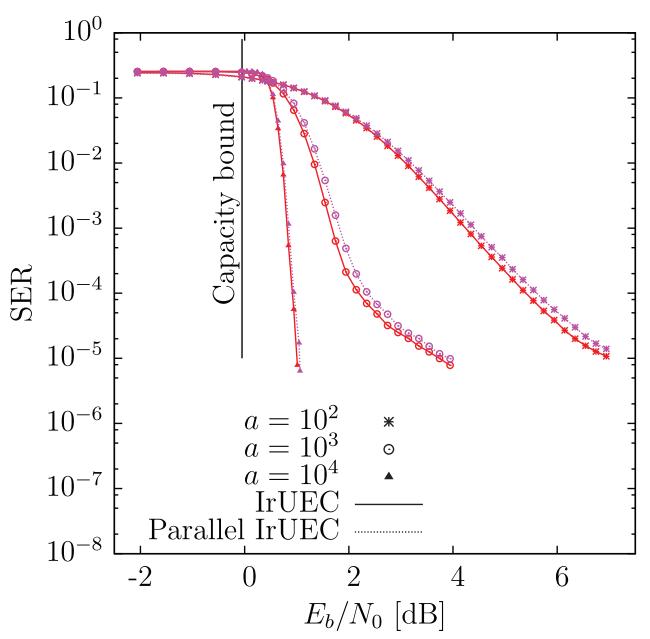

Fig. 11. SER performance for various frame lengths $a \in\left\{10^{2}, 10^{3}, 10^{4}\right\}$ of the proposed IrUEC-IrURC scheme of Fig. 1 and the Parallel IrUEC-IrURC scheme of Fig. 9, when conveying symbols obeying a zeta distribution having the parameter $p_{1}=0.797$, and communicating over a QPSK-modulated uncorrelated narrowband Rayleigh fading channel having a range of $E_{\mathrm{b}} / N_{0}$ values.

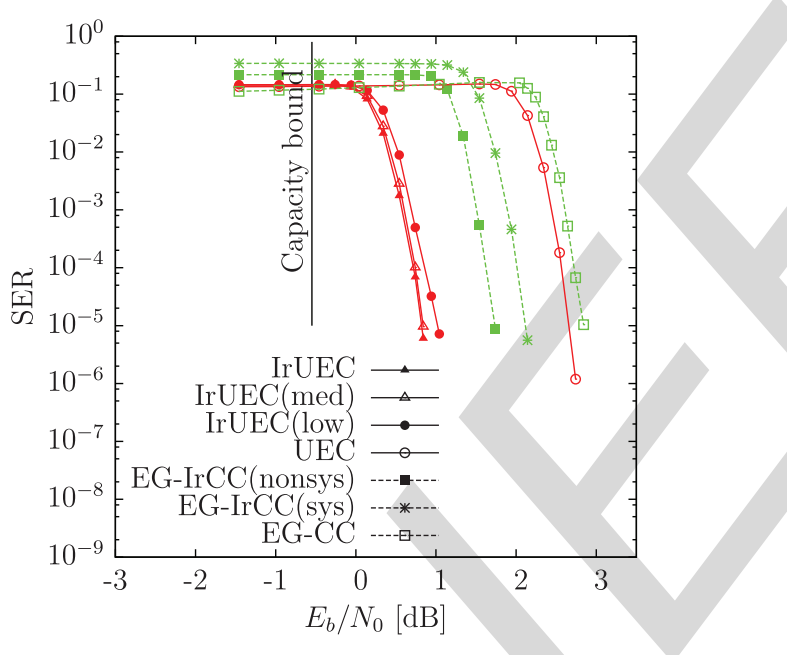

Fig. 12. SER performance for various arrangements of the proposed IrUECIrURC scheme of Fig. 1, the EG-IrCC-IrURC of Fig. 7, the Parallel IrUECIrURC scheme of Fig. 9, as well as the UEC-IrURC and the EG-IrURC schemes of [10], when conveying symbols obey a zeta distribution having the parameter $p_{1}=0.9$, and communicating over a QPSK-modulated uncorrelated narrowband Rayleigh fading channel having a range of $E_{\mathrm{b}} / N_{0}$ values. The complexity is unlimited for decoding each of the bits in $\mathbf{z}$. is provided in Fig. 12 for the various schemes considered, where the source symbols obey a zeta distribution having the parameter $p_{1}=0.9$, where the complexity is potentially unlimited. It can be seen that the proposed IrUEC-IrURC scheme also outperforms all other benchmarkers in this situation, offering a $1 \mathrm{~dB}$ gain compared to the EG-IrCC(nonsys)-IrURC scheme, which is the best-performing one of the set of SSCC benchmarkers.

Note that the performance gain of the proposed IrUECIrURC scheme is obtained by elaborately designing the IrUEC EXIT function, in order to create a narrow but marginally open EXIT chart tunnel at a low $E_{\mathrm{b}} / N_{0}$ value that is close to the area bound and capacity bound, as discussed in Section IV-B. Since the benchmarker schemes suffer from capacity loss which sep- 1012 arates their tunnel, area and capacity bounds, the performance 1013 gain of the proposed IrUEC-IrURC scheme depicted in Fig. 101014 and 12 may be expected in the general case, regardless of the 1015 specific source probability distribution and the parametrization 1016 of the scheme. As an additional benefit of the proposed IrUEC- 1017 IrURC scheme, a single bit error within a particular codeword 1018 can only result in splitting it into two codewords, or into merg- 1019 ing it with the next codeword, since every unary codeword 1020 contains only a single 0 . Fortunately, the decoding of the other 1021 unary codewords will be unaffected. Owing to this, a single bit 1022 error in the IrUEC-IrURC scheme can only cause a Levenshtein 1023 distance [33] of 2, hence preventing error propagation. By con- 1024 trast, in the EG-based benchmarkers, a single bit error can cause 1025 error propagation, resulting in a Levenshtein distance that is 1026 bounded only by the length of the message.

\section{CONCLUSIONS}

1028

In this paper, we have proposed a novel near-capacity JSCC 1029 scheme, which we refer to as the IrUEC code. Like the regular 1030 UEC code of [10], this employs a unary code, but replaces the 1031 UEC's trellis code with a novel IrTrellis code. Unlike a con- 1032 ventional irregular code, the IrTrellis code operates on the basis 1033 of a single amalgamated irregular trellis, rather than a number 1034 of separate trellises. Our results demonstrated that this single 1035 amalgamated trellis offers gains of up to $0.2 \mathrm{~dB}$ over the use 1036 of separate trellises, without imposing any increase in trans- 1037 mission energy, bandwidth, latency or decoding complexity. By 1038 characterizing the free distance property of the UEC trellis, we 1039 have selected a suite of UEC codes having a wide variety of 1040 EXIT chart shapes for the component codes of our IrUEC code. 1041 We concatenated the proposed IrUEC code with an IrURC code 1042 in Fig. 1 and introduced a new double-sided EXIT chart match- 1043 ing algorithm. On the one hand, the component UEC codes 1044 having a wide variety of EXIT chart shapes provide a great 1045 design freedom of the IrUEC EXIT chart. On the other hand, the 1046 novel double-sided EXIT chart matching algorithm utilize this 1047 design freedom sufficiently, in order to parametrize the IrUEC- 1048 IrURC scheme for creating a narrow but marginally open EXIT 1049 chart tunnel at a low $E_{\mathrm{b}} / N_{0}$ value that is close the area bound 1050 and the capacity bound. As a result, near-capacity operation 1051 is facilitated at $E_{\mathrm{b}} / N_{0}$ values that are within $0.4 \mathrm{~dB}$ of the 1052 DCMC capacity bound, when achieving an effective throughput 1053 of $\eta=0.508 \mathrm{bit} / \mathrm{s} / \mathrm{Hz}$ and employing (QPSK) for transmission 1054 over an uncorrelated narrowband Rayleigh fading channel. This 1055 corresponds to a gain of $0.8 \mathrm{~dB}$ compared to the best of several 1056 SSCC benchmarkers, which is achieved without any increase in 1057 transmission energy, bandwidth, transmit duration or decoding 1058 complexity.

\section{REFERENCES}

[1] J. Zou, H. Xiong, C. Li, R. Zhang, and Z. He, "Lifetime and distor- 1061 tion optimization with joint source/channel rate adaptation and network 1062 coding-based error control in wireless video sensor networks," IEEE 1063 Trans. Veh. Technol., vol. 60, no. 3, pp. 1182-1194, Mar. 2011.

[2] Y. Huo, C. Zhu, and L. Hanzo, "Spatio-temporal iterative source-channel 1065 decoding aided video transmission," IEEE Trans. Veh. Technol., vol. 62, 1066 no. 4, pp. 1597-1609, May 2013. 
1068

1069

1070

1071

1072

1073

1074

1075

1076

1077

1078

1079

1080

1081

1082

1083

1084

1085

1086

1087

1088

1089

1090

1091

1092

1093

1094

1095

1096

1097

1098

1099

1100

1101

1102

1103

1104

1105

1106

1107

1108

1109

1110

1111

1112

1113

1114

1115

1116

1117

1118

1119

1120

1121

1122

1123

1124

1125

1126

1127

1128

1129

1130

1131

1132

1133

1134

1135

1136

1137

1138

1139

1140
[3] N. S. Othman, M. El-Hajjar, O. Alamri, S. X. Ng, and L. Hanzo, "Iterative AMR-WB source and channel decoding using differential space-time spreading-assisted sphere-packing modulation," IEEE Trans. Veh. Technol., vol. 58, no. 1, pp. 484-490, Jan. 2009.

[4] C. E. Shannon, "The mathematical theory of communication," Bell Syst. Tech. J., vol. 27, pp. 379-423, Jul. 1948.

[5] B. Ryabko and J. Rissanen, "Fast adaptive arithmetic code for large alphabet sources with asymmetrical distributions," in Proc. IEEE Int. Symp. Inf. Theory, 2002, p. 319.

[6] J. Ziv and A. Lempel, "Compression of individual sequences via variable rate coding," IEEE Trans. Inf. Theory, vol. 24, no. 5, pp. 530-536, Sep. 1978.

[7] P. Elias, "Universal codeword sets and representations of the integers," IEEE Trans. Inf. Theory, vol. 21, no. 2, pp. 194-203, Mar. 1975.

[8] Advanced Video Coding for Generic Audiovisual Services, ITU-T Std. H.264, Mar. 2005.

[9] J. L. Massey, "Joint source and channel coding," in Proc. Commun. Syst. Random Process Theory, Dec. 1978, pp. 279-293.

[10] R. G. Maunder, W. Zhang, T. Wang, and L. Hanzo, "A unary error correction code for the near-capacity joint source and channel coding of symbol values from an infinite set," IEEE Trans. Commun., vol. 61, no. 5, pp. 1977-1987, May 2013

[11] N. L. Johnson, A. W. Kemp, and S. Kotz, Univariate Discrete Distributions. Hoboken, NJ, USA: Wiley, 2005.

[12] Y. Takishima, M. Wada, and H. Murakami, "Reversible variable length codes," IEEE Trans. Commun., vol. 43, no. 234, pp. 158-162, Feb. 1995.

[13] V. Buttigieg and P. G. Farrell, "Variable-length error-correcting codes," IEE Proc. Commun., vol. 147, no. 4, pp. 211-215, Aug. 2000.

[14] R. G. Maunder and L. Hanzo, "Near-capacity irregular variable length coding and irregular unity rate coding," IEEE Trans. Wireless Commun., vol. 8, no. 11, pp. 5500-5507, Nov. 2009.

[15] M. Tüchler, "Design of serially concatenated systems depending on the block length," IEEE Trans. Commun., vol. 52, no. 2, pp. 209-218, Feb. 2004

[16] R. G. Maunder and L. Hanzo, "Genetic algorithm aided design of component codes for irregular variable length coding," IEEE Trans. Commun., vol. 57, no. 5, pp. 1290-1297, May 2009.

[17] A. Ashikhmin, G. Kramer, and S. ten Brink, "Code rate and the area under extrinsic information transfer curves," in Proc. IEEE Int. Symp. Inf. Theory, Lausanne, Switzerland, Jun. 2002, p. 115.

[18] W. Zhang, R. G. Maunder, and L. Hanzo, "On the complexity of unary error correction codes for the near-capacity transmission of symbol values from an infinite set," in Proc. IEEE Wireless Commun. Netw. Conf., Apr. 2013, pp. 2795-2800.

[19] T. Wang, W. Zhang, R. G. Maunder, and L. Hanzo, "Near-capacity joint source and channel coding of symbol values from an infinite source set using Elias gamma error correction codes," IEEE Trans. Commun., vol. 62, no. 1, pp. 280-292, Jan. 2014

[20] L. Hanzo, R. G. Maunder, J. Wang, and L.-L. Yang, Near-Capacity Variable Length Coding. Hoboken, NJ, USA: Wiley, 2010.

[21] S. Benedetto and G. Montorsi, "Iterative decoding of serially concatenated convolutional codes," Electron. Lett., vol. 32, no. 13, pp. 11861188, Jun. 1996

[22] L. Bahl, J. Cocke, F. Jelinek, and J. Raviv, "Optimal decoding of linear codes for minimizing symbol error rate," IEEE Trans. Inf. Theory, vol. 20, no. 2, pp. 284-287, Mar. 1974

[23] R. G. Maunder and L. Hanzo, "Iterative decoding convergence and termination of serially concatenated codes," IEEE Trans. Veh. Technol., vol. 59, no. 1, pp. 216-224, Jan. 2010.

[24] J. Kliewer, N. Goertz, and A. Mertins, "Iterative source-channel decoding with Markov random field source models," IEEE Trans. Signal Process., vol. 54 , no. 10 , pp. 3688-3701, Oct. 2006

[25] D. Divsalar, H. Jin, and R. J. McEliece, "Coding theorems for turbo like codes," in Proc. 36th Allerton Conf. Commun. Control Comput., Allerton House, IA, USA, Sep. 1998, pp. 201-210.

[26] A. Diallo, C. Weidmann, and M. Kieffer, "Efficient computation and optimization of the free distance of variable-length finite-state joint sourcechannel codes," IEEE Trans. Commun., vol. 59, no. 4, pp. 1043-1052, Apr. 2011.

[27] S. ten Brink, "Convergence behavior of iteratively decoded parallel concatenated codes," IEEE Trans. Commun., vol. 49, no. 10, pp. 1727-1737, Oct. 2001.
[28] M. Tüchler and J. Hagenauer, "EXIT charts of irregular codes," in Proc. 1141 Conf. Inf. Sci. Syst., Princeton, NJ, USA, Mar. 2002, pp. 748-753.

[29] L. Hanzo, T. H. Liew, B. L. Yeap, R. Y. S. Tee, and S. X. Ng, Turbo 1143 Coding, Turbo Equalisation and Space-Time Coding: EXIT-Chart Aided 1144 Near-Capacity Designs for Wireless Channels. Hoboken, NJ, USA: 1145 Wiley, 2010.

[30] J. Kliewer, A. Huebner, and D. J. Costello, "On the achievable extrinsic 1147 information of inner decoders in serial concatenation," in Proc. IEEE Int. 1148 Symp. Inf. Theory, Seattle, WA, USA, Jul. 2006, pp. 2680-2684. 1149

[31] P. Frenger, P. Orten, and T. Ottosson, "Convolutional codes with optimum 1150 distance spectrum," IEEE Commun. Lett., vol. 3, no. 11, pp. 317-319, 1151 Nov. 1999.

1152

[32] L. Li, R. G. Maunder, B. M. Al-Hashimi, and L. Hanzo, "A low- 1153 complexity turbo decoder architecture for energy-efficient wireless sensor 1154 networks," IEEE Trans. Very Large Scale Integr. (VLSI) Syst., vol. 21, 1155 no. 1, pp. 14-22, Jan. 2013

[33] D. Sankoff and J. B. Kruskal, Time Warps, String Edits, and 1157 Macromolecules: The Theory and Practice of Sequence Comparison. 1158 Reading, MA, USA: Addison-Wesley, 1983.



Wenbo Zhang (S'14) received the M.E. degree in 1160 information and communication engineering from 1161 the University of Beijing University of Posts and 1162 Telecommunications (BUPT), Beijing, China, in 1163 2011. Currently, he is pursuing the Ph.D. degree 1164 at the Communications Research Group, School of 1165 Electronics and Computer Science, University of 1166 Southampton, Southampton, U.K. His research inter- 1167 ests include joint source/channel coding and variable 1168 length coding.

1169

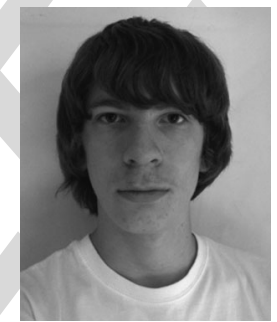

Matthew F. Brejza received the B.Eng. degree 1170 (first class Hons.) in electronic engineering from the 1171 University of Southampton, Southampton, U.K., in 1172 2012, and is currently pursuing the Ph.D. degree 1173 at the Communications Research Group, School of 1174 Electronics and Computer Science, University of 1175 Southampton. His research interests include flexible 1176 hardware implementation, channel coding and their 1177 applications in low power data communications.

1178



Tao Wang received the B.S. degree in informa- 1179 tion engineering from the University of Science 1180 and Technology of Beijing (USTB), Beijing, China, 1181 and the M.Sc. degree in communication from 1182 University of Southampton, Southampton, U.K., in 1183 2006 and 2008, respectively, and is currently pursu- 1184 ing the Ph.D. degree at the Communications Research 1185 Group, School of Electronics and Computer Science, 1186 University of Southampton. His research interests 1187 include joint source/channel coding and distributed 1188 video coding.

1189



Robert G. Maunder (SM'12) received the B.Eng. 1190 degree (first class Hons.) in electronic engineering 1191 and the Ph.D. degree in wireless communications, 1192 in 2003 and 2007, respectively. He has authored a 1193 number of IEEE papers in his research interest areas. 1194 His research interests include joint source/channel 1195 coding, iterative decoding, irregular coding, and mod- 1196 ulation techniques. He received a lectureship in 1197 December 2007. 




Lajos Hanzo (F'04) received the degree in electronics and the doctorate degree, in 1976 and 1983, respectively. During his 38-year career in telecommunications, he has held various research and academic posts in Hungary, Germany, and the U.K. Since 1986, he has been with the School of Electronics and Computer Science, University of Southampton, Southampton, U.K., where he holds the Chair in telecommunications. He has coauthored 20 Wiley/IEEE Press books on mobile radio communications totalling in excess of 10000 pages, authored more than 1500 research entries at the IEEE Xplore, acted both as TPC and General Chair of the IEEE conferences, presented keynote lectures and has been awarded a number of distinctions. Currently, he is directing a 60strong academic research team, working on a range of research projects in the field of wireless multimedia communications sponsored by industry, the Engineering and Physical Sciences Research Council (EPSRC), U.K., the European Research Council's Advanced Fellow Grant, and the Royal Society's Wolfson Research Merit Award. He is a Governor of the IEEE VTS. From 2008 to 2012, he was the Editor-in-Chief of the IEEE Press and also a Chaired Professor at Tsinghua University, Beijing, China. His research is funded by the European Research Council's Senior Research Fellow Grant. Lajos has more than 22000 citations. In 2009, he was was the recipient of an Honorary Doctorate by the Technical University of Budapest, while in 2015, by the University of Edinburgh. 


\title{
Irregular Trellis for the Near-Capacity Unary Error Correction Coding of Symbol Values From an Infinite Set
}

\author{
Wenbo Zhang, Student Member, IEEE, Matthew F. Brejza, Tao Wang, Robert G. Maunder, Senior Member, IEEE, \\ and Lajos Hanzo, Fellow, IEEE
}

Abstract-Irregular joint source and channel coding (JSCC) scheme is proposed, which we refer to as the irregular unary error correction (IrUEC) code. This code operates on the basis of a single irregular trellis, instead of employing a set of separate regular trellises, as in previous irregular trellis-based codes. Our irregular trellis is designed with consideration of the UEC free distance, which we characterize for the first time in this paper. We conceive the serial concatenation of the proposed IrUEC code with an irregular unity rate code (IrURC) code and propose a new EXtrinsic Information Transfer (EXIT) chart matching algorithm for parametrizing these codes. This facilitates the creation of a narrow EXIT tunnel at a low $E_{\mathrm{b}} / N_{0}$ value and provides near-capacity operation. Owing to this, our scheme is found to offer a low symbol error ratio (SER), which is within $0.4 \mathrm{~dB}$ of the discrete-input continuous-output memoryless channel (DCMC) capacity bound in a particular practical scenario, where gray-mapped quaternary phase shift keying (QPSK) modulation is employed for transmission over an uncorrelated narrowband Rayleigh-fading channel with an effective throughput of $0.508 \mathrm{bits}^{-1} \mathrm{~Hz}^{-1}$. Furthermore, the proposed IrUEC-IrURC scheme offers a SER performance gain of $0.8 \mathrm{~dB}$, compared to the best of several regular and irregular separate source and channel coding (SSCC) benchmarkers, which is achieved without any increase in transmission energy, bandwidth, transmit duration, or decoding complexity.

Index Terms-Joint source-channel coding, irregular codecs, channel capacity, iterative decoding.

\section{INTRODUCTION}

N MOBILE wireless scenarios, multimedia transmission is required to be bandwidth efficient and resilient to transmission errors, motivating both source and channel coding [1]-[3]. Classic Separate Source and Channel Coding (SSCC) may be achieved by combining a near-entropy source code with a near-capacity channel code. In this scenario, it is theoretically

Manuscript received October 31, 2014; revised April 14, 2015 and July 25, 2015; accepted October 8, 2015. This work was supported in part by the EPSRC, Swindon UK under Grant EP/J015520/1 and Grant EP/L010550/1, in part by the TSB Swindon UK under Grant TS/L009390/1, in part by the RCUK under the India-UK Advanced Technology Centre (IU-ARC), and in part by the EU under the CONCERTO project and in part by the European Research Council's Advanced Fellow grant. The associate editor coordinating the review of this paper and approving it for publication was M. Xiao.

The authors are with School of Electronics and Computer Science, University of Southampton, Southampton SO17 1BJ, U.K. (e-mail: wz4g11@ecs.soton. ac.uk; mfb2g09@ecs.soton.ac.uk; tw08r@ecs.soton.ac.uk; rm@ecs.soton. ac.uk; lh@ecs.soton.ac.uk).

Color versions of one or more of the figures in this paper are available online at http://ieeexplore.iee.org.

Digital Object Identifier 10.1109/TCOMM.2015.2493149 possible to reconstruct the source information with an infinitesimally low probability of error, provided that the transmission rate does not exceed the channel's capacity [4]. However, separate source-channel coding [4] is only capable of approaching the capacity in the general case by imposing both infinite complexity and infinite latency. For example, adaptive arithmetic coding [5] and Lempel-Ziv coding [6] are capable of encoding a sequence of symbols using a near-entropy number of bits per symbol. However, these schemes require both the transmitter and receiver to accurately estimate the occurrence probability of every symbol value that the source produces. In practice, the occurrence probability of rare symbol values can only be accurately estimated, if a sufficiently large number of symbols has been observed, hence potentially imposing an excessive latency.

This motivates the design of universal codes, such as the Elias Gamma (EG) code [7], which facilitate the binary encoding of symbols selected from infinite sets, without requiring any knowledge of the corresponding occurrence probabilities at either the transmitter or receiver. The H.264 video codec [8] employs the EG code and this may be concatenated with classic channel codes, such as a Convolutional Code (CC) to provide a separate error correction capability. Nevertheless, this SSCC typically suffers from a capacity loss, owing to the residual redundancy that is typically retained during EG encoding, which results in an average number of EG-encoded bits per symbol that exceeds the entropy of the symbols.

In order to exploit the residual redundancy and hence to achieve near-capacity operation, the classic SSCC schemes may be replaced by Joint Source and Channel Coding (JSCC) arrangements [9] in many applications. As we have previously demonstrated in [10, Fig. 1], the symbols that are EG encoded in H.264 are approximately zeta probability distributed [11], resulting in most symbols having low values, but some rare symbols having values around 1000 . Until recently, the decoding complexity of all previous JSCCs, such as Reversible Variable Length Codes (RVLCs) [12] and Variable Length Error Correction (VLEC) codes [13], increased rapidly with the cardinality of the symbol set, so much so that it became excessive for the H.264 symbol probability distribution and asymptotically tending to infinity, when the cardinality is infinite.

Against this background, a novel JSCC scheme referred to as a Unary Error Correction (UEC) code [10] was proposed as the first JSCC that mitigates the capacity loss and incurs 

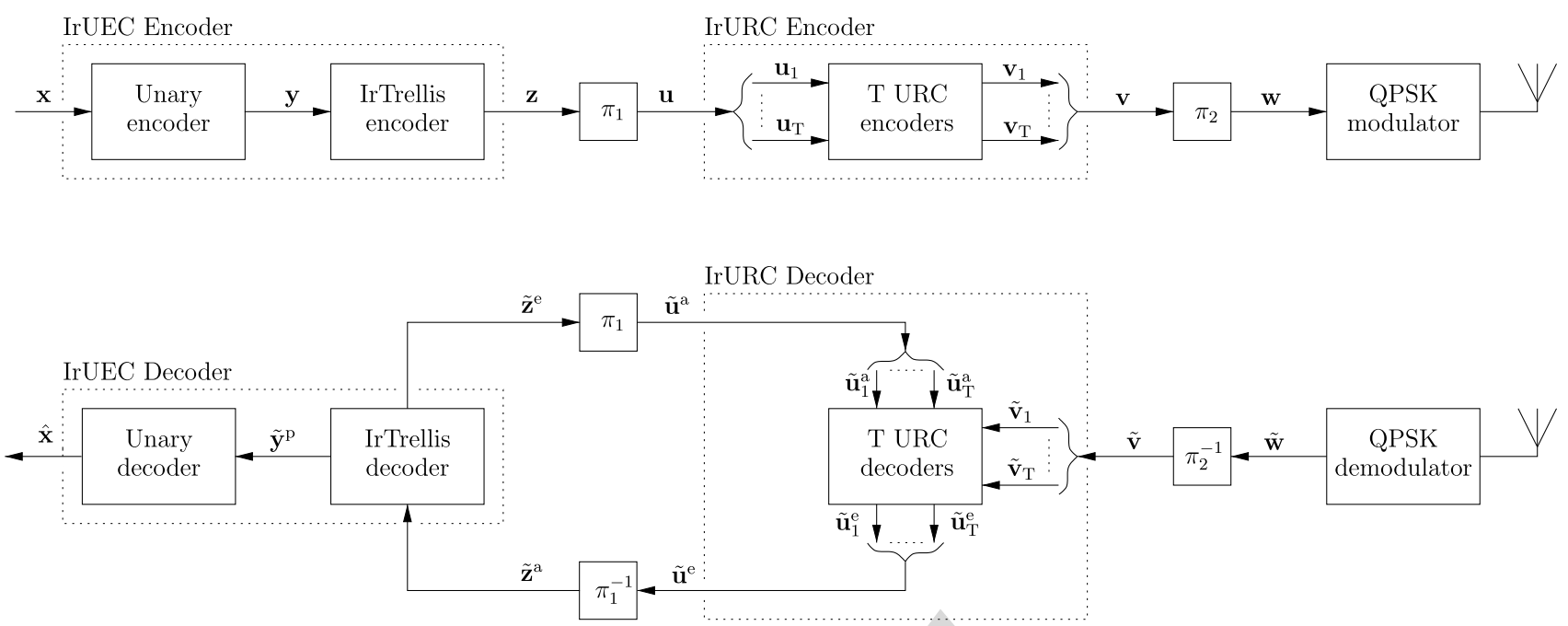

Fig. 1. Schematic of the proposed IrUEC-IrURC scheme, in which an IrUEC code is serially concatenated with IrURC code and Gray-coded QPSK modulation schemes. Here, $\pi_{1}$ and $\pi_{2}$ represent interleavers, while $\pi_{1}^{-1}$ and $\pi_{2}^{-1}$ represent the corresponding deinterleavers.

only a moderate decoding complexity, even when the cardinality of the symbol set is infinite. In a particular practical scenario, an iteratively-decoded serial concatenation of the UEC code with an Irregular Unity Rate Code (IrURC) was shown to offer a $1.3 \mathrm{~dB}$ gain compared to a SSCC benchmarker, without incurring an increased transmission energy, duration, bandwidth or decoding complexity. Furthermore, this was achieved within $1.6 \mathrm{~dB}$ of the Quaternary Phase Shift Keying (QPSK)-modulated uncorrelated narrow band Rayleigh fading Discrete-input Continuous-output Memoryless Channel (DCMC) capacity bound.

In this paper, we will further exploit the properties of UEC codes in order to facilitate reliable operation even closer to the capacity bound. More specifically, we propose an Irregular Unary Error Correction (IrUEC) code, which extends the regular UEC of our previous work [10]. This IrUEC code employs different UEC parametrizations for the coding of different subsets of each message frame, in analogy with previous irregular codes, such as the IrURC [14], the Irregular Convolutional Code (IrCC) [15] and the Irregular Variable Length Code (IrVLC) [16]. However, these previous irregular codes operate on the basis of a number of separate trellises, each of which has a different but uniform structure and is used for the coding of a different subset of the message frame. By contrast, our new IrUEC code operates on the basis of a single irregular trellis having a novel design. This trellis has a non-uniform structure that applies different UEC parametrizations for different subsets of the frame on a bit-by-bit basis. This allows the irregularity of the proposed IrUEC code to be controlled on a fine-grained bit-by-bit basis, rather than on a symbol-bysymbol basis, hence facilitating nearer-to-capacity operation. More specifically, our results demonstrate that controlling the IrUEC irregularity on a bit-by-bit basis offers gains of up to $0.2 \mathrm{~dB}$ over the symbol-by-symbol approach, without imposing any increase in transmission energy, bandwidth, latency or decoding complexity.

This bit-by-bit IrUEC approach is facilitated by some particular properties of UEC codes, which grant some commonality to all UEC parametrizations. By exploiting this fine-grained 117 control of the IrUEC irregularity, the IrUEC EXtrinsic 118 Information Transfer (EXIT) function may be shaped to cre- 119 ate a narrow, but marginally open EXIT chart tunnel. This 120 implies that near-capacity operation is facilitated, according to 121 the theoretical properties of EXIT charts [17].

The rest of this paper is organized as follows. Section II 123 describes a transmitter that serially concatenates the proposed 124 IrUEC encoder with a IrURC encoder, while Section III 125 describes the corresponding iterative receiver. The IrUEC 126 encoder and decoder operate on the basis of our novel irregular 127 trellis structure, which allows bit-level control of the irregular 128 coding fractions. The free distance of UEC codes is quantified 129 for the first time in Section IV, which proposes a novel low- 130 complexity heuristic method conceived for this purpose. This is 131 used for selecting a family of UEC trellis structures having a 132 wide variety of EXIT function shapes. The resultant UEC trel- 133 lis family maximises the design freedom for the IrUEC EXIT 134 function and therefore has a general applicability for IrUEC 135 codes used in diverse applications. Furthermore, for any partic- 136 ular application of an IrUEC code, we propose a double-sided 137 EXIT chart matching algorithm for selecting the specific frac- 138 tion of the frame that should be encoded using each IrUEC 139 and IrURC trellis structure. This allows the EXIT functions of 140 IrUEC and IrURC codes to be accurately shaped for closely 141 matching each other, hence creating a narrow but marginally 142 open EXIT chart tunnel. In Section V, the proposed IrUEC- 143 IrURC scheme is compared to an irregular JSCC benchmarker, 144 which is referred to as the EG-IrCC-IrURC scheme. The first 145 version of this benchmarker employs the recursive systematic 146 CCs that were originally recommended as IrCC component 147 codes in [15]. However, we demonstrate that the systematic 148 nature of these IrCC component codes results in a capacity loss. 149 This motivates the employment of the second version of our 150 EG-IrCC-IrURC benchmarker, which employs the recursive 151 non-systematic CCs of [10] as the IrCC component codes. The 152 simulation results of Section V show that in a particular prac- 153 tical scenario, the proposed IrUEC-IrURC scheme provides a 154 
$0.8 \mathrm{~dB}$ gain over the best SSCC benchmarker, while operating within $0.4 \mathrm{~dB}$ of the capacity bound. This is achieved without any increase in transmission energy, bandwidth, latency or decoding complexity. Finally, Section VI concludes the paper.

\section{IRUEC-IRURC ENCODER}

In this section, we introduce the transmitter of the proposed IrUEC-IrURC scheme of Fig. 1. The IrURC encoder employs $T$ number of component Unity Rate Code (URC) encoders $\left\{\mathrm{URC}^{t}\right\}_{t=1}^{T}$, each having a distinct independent trellis structure. By contrast, the IrUEC employs a unary encoder and a novel Irregular Trellis (IrTrellis) encoder with a single irregular trellis. However, in analogy with the IrURC code, we note that this irregular trellis comprises a merging of $S$ component UEC trellis structures $\left\{\mathrm{UEC}^{s}\right\}_{s=1}^{S}$, where $\mathrm{UEC}^{s}$ is the $s$-th component UEC trellis structure that is defined by the corresponding codeword set $\mathbb{C}_{s}$, as illustrated in [10, Fig. 3(a)]. In Section II-A and Section II-B, the two components of the IrUEC encoder in Fig. 1, namely the unary encoder and the novel IrTrellis encoder are detailed. The IrURC encoder and the modulator are introduced in Section II-C.

\section{A. Unary Encoder}

The IrUEC encoder is designed for conveying a vector $\mathbf{x}=\left[x_{i}\right]_{i=1}^{a}$ comprising $a$ number of symbols, as shown in Fig. 1. The value of each symbol $x_{i} \in \mathbb{N}_{1}$ may be modeled by an Independent and Identically Distributed (IID) Random Variable $(\mathrm{RV}) X_{i}$, which adopts the value $x$ with a probability of $\operatorname{Pr}\left(X_{i}=x\right)=P(x)$, where $\mathbb{N}_{1}=\{1,2,3, \ldots, \infty\}$ is the infinite-cardinality set comprising all positive integers. Throughout this paper we assume that the symbol values obey a zeta probability distribution [11], since this models the symbols produced by multimedia encoders, as described in Section I. The zeta probability distribution is defined as

$$
P(x)=\frac{x^{-s}}{\zeta(s)}
$$

where $\zeta(s)=\sum_{x \in \mathbb{N}_{1}} x^{-s}$ is the Riemann zeta function, $s>1$ parametrizes the zeta distribution and $p_{1}=\operatorname{Pr}\left(X_{i}=1\right)=$ $1 / \zeta(s)$ is the probability of occurrence for the most frequently occurring symbol value, namely $x=1$. Without loss of generality, Table I exemplifies the first ten symbol probabilities $P\left(x_{i}\right)$ for a zeta distribution having the parameter $p_{1}=0.797$, which corresponds to $s=2.77$ and was found in [10] to allow a fair comparison between unary- and EG-based schemes. Note that other $p_{1}$ values of $0.694,0.8$ and 0.9 have been investigated in [18], [19]. In the situation where the symbols obey the zeta probability distribution of (1), the symbol entropy is given by

$$
H_{X}=\sum_{x \in \mathbb{N}_{1}} H[P(x)]=\frac{\ln (\zeta(s))}{\ln (2)}-\frac{s \zeta^{\prime}(s)}{\ln (2) \zeta(s)},
$$

where $H[p]=p \log _{2}(1 / p)$ and $\zeta^{\prime}(s)=-\sum_{x \in \mathbb{N}_{1}} \ln (x) x^{-s}$ is the derivative of the Riemann zeta function.

As shown in Fig. 1, the IrUEC encoder represents the source vector $\mathbf{x}$ using a unary encoder. More specifically, each symbol
TABLE I

The First TEN Symbol Probabilities FOR A ZETA Distribution HAVING THE PARAMETER $p_{1}=0.797$, AS WELL AS THE CORRESPONDING UNARY AND EG CODEWORDS

\begin{tabular}{|c|l|l|l|}
\hline \multirow{2}{*}{$x_{i}$} & \multirow{2}{*}{$P\left(x_{i}\right)$} & \multicolumn{2}{|c|}{$\mathbf{y}_{i}$} \\
\cline { 3 - 4 } & & \multicolumn{1}{|c|}{ Unary } & EG \\
\hline 1 & 0.797 & 0 & 1 \\
\hline 2 & 0.117 & 10 & 010 \\
\hline 3 & 0.038 & 110 & 011 \\
\hline 4 & 0.017 & 1110 & 00100 \\
\hline 5 & 0.009 & 11110 & 00101 \\
\hline 6 & 0.006 & 111110 & 00110 \\
\hline 7 & 0.004 & 1111110 & 00111 \\
\hline 8 & 0.003 & 11111110 & 0001000 \\
\hline 9 & 0.002 & 111111110 & 0001001 \\
\hline 10 & 0.001 & 1111111110 & 0001010 \\
\hline
\end{tabular}

$x_{i}$ in the vector $\mathbf{x}$ is represented by a corresponding codeword 202 $\mathbf{y}_{i}$ that comprises $x_{i}$ bits, namely $\left(x_{i}-1\right)$ binary ones followed 203 by a zero, as exemplified in Table I. When the symbols adopt 204 the zeta distribution of (1), the average unary codeword length $l 205$ is only finite for $s>2$ and hence for $p_{1}>0.608$ [10], in which 206 case we have

$$
l=\sum_{x \in \mathbb{N}_{1}} P(x) \cdot x=\frac{\zeta(s-1)}{\zeta(s)} .
$$

Note that for $p_{1} \leq 0.608$, our Elias Gamma Error Correction 208 (EGEC) code of [19] may be employed in order to achieve a 209 finite average codeword length, albeit at the cost of an increased 210 complexity. In our future work, we will consider a novel 211 Irregular EGEC code, which has a finite codeword length for 212 $p_{1} \leq 0.608$. Without loss of generality, in the example scenario 213 of $p_{1}=0.797$, an average codeword length of $l=1.54$ results. 214 The output of the unary encoder is generated by concatenating 215 the selected codewords $\left\{\mathbf{y}_{i}\right\}_{i=1}^{a}$, in order to form the $b$-bit vec- 216 tor $\mathbf{y}=\left[y_{j}\right]_{j=1}^{b}$. For example, the source vector $\mathbf{x}=[4,1,2]$ of 217 $a=3$ symbols yields the $b=7$-bit vector $\mathbf{y}=[1110010]$. Note 218 that the average length of the bit vector $\mathbf{y}$ is given by $(a \cdot l)$.

\section{B. IrTrellis Encoder}

Following unary encoding, the IrTrellis encoder of Fig. 1221 employs a single new irregular trellis to encode the bit vec- 222 tor $\mathbf{y}$, rather than using a selection of separate trellis structures, 223 as is necessary for the IrCC [15], IrVLC [16] and IrURC [14] 224 coding schemes. Our novel irregular trellis structure is facil- 225 itated by the properties of the generalised trellis structure of 226 [10, Fig. 3(a)], which was the basis of our previous work on 227 regular UEC codes. This trellis structure is parametrized by 228 an even number of states $r$ and by the UEC codeword set $\mathbb{C}, 229$ which comprises $r / 2$ binary codewords of a particular length 230 $n$. Each bit $y_{j}$ of the unary-encoded bit sequence $\mathbf{y}=\left[y_{j}\right]_{j=1}^{b} 231$ corresponds to a transition in the UEC trellis from the previous 232 state $m_{j-1} \in\{1,2, \ldots, r\}$ to the next state $m_{j} \in\{1,2, \ldots, r\} .233$ Each next state $m_{j}$ is selected from two legitimate alternatives, 234 depending both on the previous state $m_{j-1}$ and on the bit value 235 $y_{j}$, according to [18, (3)]. More specifically, regardless of how 236 the UEC trellis is parametrized, a unary-coded bit of $y_{j}=1237$ causes a transition towards state $m_{j}=r-1$ or $r$ of the gener- 238 alised UEC trellis of [10, Fig. 3(a)], while the $y_{j}=0$-valued bit 239 
at the end of each unary codeword causes a transition to state $m_{j}=1$ or 2 , depending on whether the current symbol $x_{i}$ has an odd or even index $i$.

This common feature of all UEC trellises maintains synchronisation with the unary codewords and allows the residual redundancy that remains following unary encoding to be explicated for error correction. Furthermore, this common treatment of the unary-encoded bits in $\mathbf{y}$ between all UEC trellises allows them to merge in order to form our novel irregular trellis. More specifically, our novel irregular trellis can be seen as concatenation of a number of individual UEC trellis structures with different numbers of states $r$ and different codeword sets $\mathbb{C}$. By contrast, CCs, Variable Length Codes (VLCs) and URC codes having different parametrizations do not generally exhibit the required similarity in their trellises. More specifically, the final state of a particular component encoder has no specific relationship with the initial state of the subsequent component encoder, hence preventing their amalgamation into IrCC, IrVLC and IrURC trellises, respectively.

The IrTrellis encoder of Fig. 1 encodes the $b$-bit unaryencoded bit sequence $\mathbf{y}=\left[y_{j}\right]_{j=1}^{b}$ using an irregular trellis that is obtained by concatenating $b$ number of regular UEC trellis structures. The proposed IrTrellis can be constructed using diverse combinations of component regular UEC trellises, having any parametrization. However, the component regular trellises may be strategically selected in order to carefully shape the EXIT function of the IrUEC code, for the sake of producing a narrow EXIT chart tunnel and for facilitating near-capacity operation, as it will be detailed in Section IV. Without loss of generality, Fig. 1 provides an example of the irregular trellis for the example scenario where we have $b=7$. Each bit $y_{j}$ in the vector $\mathbf{y}$ is encoded using the corresponding one of these $b$ trellis structures, which is parametrized by an even number of states $r_{j}$ and the codeword set $\mathbb{C}_{j}=$ $\left\{\mathbf{c}_{1}^{j}, \mathbf{c}_{2}^{j}, \ldots, \mathbf{c}_{r_{j} / 2-1}^{j}, \mathbf{c}_{r_{j} / 2}^{j}\right\}$, which comprises $r_{j} / 2$ binary codewords of a particular length $n_{j}$. Note that successive trellis structures can have different numbers of states, subject to the constraint $r_{j} \leq r_{j-1}+2$, as it will be demonstrated in the following discussions. Note that this constraint does not restrict the generality of the IrUEC trellis, since the IrUEC EXIT function shape is independent of the ordering of the component trellis structures.
As in the regular UEC trellis of [10], the encoding process 282 always emerges from the state $m_{0}=1$. The unary-encoded 283 bits of $\mathbf{y}$ are considered in order of increasing index $j$ and 284 each bit $y_{j}$ causes the novel IrTrellis to traverse from the 285 previous state $m_{j-1} \in\left\{1,2, \ldots, r_{j-1}\right\}$ to the next state $m_{j} \in 286$ $\left\{1,2, \ldots, r_{j}\right\}$, which is selected from two legitimate alterna- 287 tives. More specifically,

$$
m_{j}=\left\{\begin{array}{ll}
1+\operatorname{odd}\left(m_{j-1}\right) & \text { if } y_{j}=0 \\
\min \left[m_{j-1}+2, r_{j}-\operatorname{odd}\left(m_{j-1}\right)\right] & \text { if } y_{j}=1
\end{array},\right.
$$

where the function odd $(\cdot)$ yields 1 if the operand is odd or 0 if 289 it is even. Note that the next state $m_{j}$ in the irregular trellis is 290 confined by the number of states $r_{j}$ in the corresponding trellis 291 structure, rather than by a constant number of states $r$, as in the 292 regular UEC trellis of [10]. In this way, the bit sequence $\mathbf{y}$ iden- 293 tifies a path through the single irregular trellis, which may be 294 represented by a vector $\mathbf{m}=\left[m_{j}\right]_{j=0}^{b}$ comprising $b+1$ state 295 values. As in the regular UEC trellis of [10], the transitions of 296 the proposed irregular trellis are synchronous with the unary 297 codewords of Table I. More specifically, just as each symbol 298 $x_{i}$ in the vector $\mathbf{x}$ corresponds to an $x_{i}$-bit codeword $\mathbf{y}_{i}$ in the 299 vector $\mathbf{y}$, the symbol $x_{i}$ also corresponds to a section $\mathbf{m}_{i}$ of 300 the trellis path $\mathbf{m}$ comprising $x_{i}$ transitions between $\left(x_{i}+1\right) \quad 301$ states. Owing to this, the path $\mathbf{m}$ is guaranteed to terminate 302 in the state $m_{b}=1$, when the symbol vector $\mathbf{x}$ has an even 303 length $a$, while $m_{b}=2$ is guaranteed when $a$ is odd [10]. Note 304 that the example unary-encoded bit sequence $\mathbf{y}=[1110010] 305$ corresponds to the path $\mathbf{m}=[1,3,5,3,2,1,1,2]$ through the 306 irregular UEC trellis of Fig. 2.

The path $\mathbf{m}$ may be modeled as a particular realization 308 of a vector $\mathbf{M}=\left[M_{j}\right]_{j=0}^{b}$ comprising $(b+1) \mathrm{RVs}$. Note that 309 the probability $\operatorname{Pr}\left(M_{j}=m_{j}, M_{j-1}=m_{j-1}\right)=P\left(m_{j}, m_{j-1}\right) \quad 310$ of the transition from the previous state $m_{j-1}$ to the next state 311 $m_{j}$ can be derived by observing the value of each symbol in 312 the vector $\mathbf{x}$ and simultaneously its corresponding index. The 313 state transition $\mathbf{M}=\left\{M_{j}\right\}_{j=0}^{b}$ follows the same rule shown in 314 (4), and all the transitions can be categorised into four types, as 315 illustrated in $[10,(8)]$. Owing to this, the probability of a tran- 316 sition $P\left(m_{j}, m_{j-1}\right)$ in the irregular trellis is associated with the 317 transition probabilities $\operatorname{Pr}\left(M_{j}=m, M_{j-1}=m^{\prime}\right)=P\left(m, m^{\prime}\right) \quad 318$ in (5), shown at the bottom of the page. Note that these 319

$$
\begin{aligned}
& \text { if } m_{j-1} \in\left\{1,2,3, \ldots, r_{j-1}-2\right\}, m_{j}=m_{j-1}+2 \\
& \text { if } m_{j-1} \in\left\{1,2,3, \ldots, r_{j-1}-2\right\}, m_{j}=1+\operatorname{odd}\left(m_{j-1}\right) \\
& \text { if } m_{j-1} \in\left\{r_{j-1}-1, r_{j-1}\right\}, m_{j}=1+\operatorname{odd}\left(m_{j-1}\right) \\
& \text { if } m_{j-1} \in\left\{r_{j-1}-1, r_{j-1}\right\}, m_{j} \in\left\{r_{j}-1, r_{j}\right\} \\
& \text { otherwise }
\end{aligned}
$$




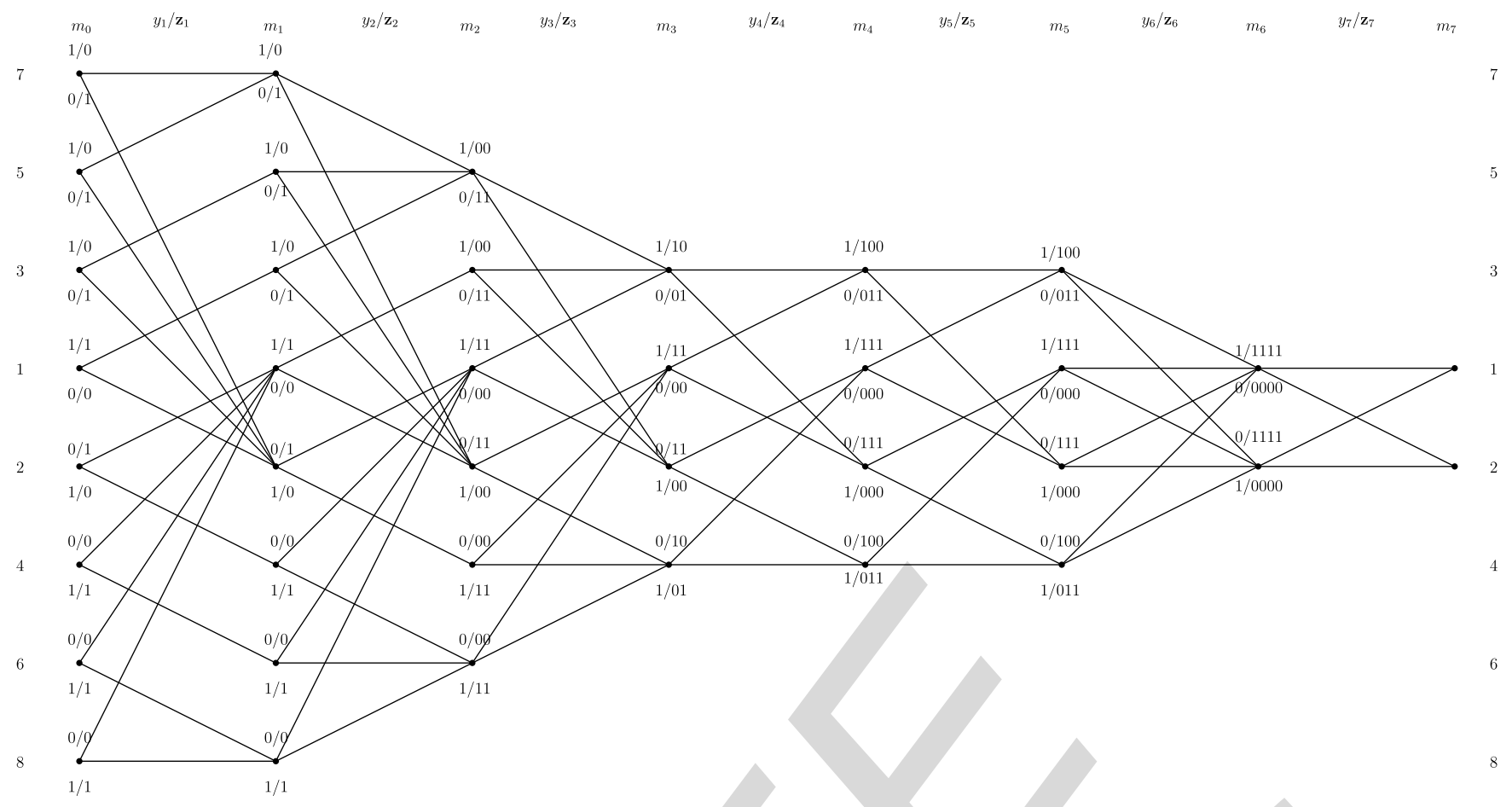

Fig. 2. An example of the proposed irregular UEC trellis, which is obtained by amalgamating seven different UEC trellises. Here, the component UEC codebooks $\mathbb{C}_{1}=\{0,1,1,1\}, \mathbb{C}_{2}=\{0,1,1,1\}, \mathbb{C}_{3}=\{000,000,000\}, \mathbb{C}_{4}=\{00,01\}, \mathbb{C}_{5}=\{000,011\}, \mathbb{C}_{6}=\{000,011\}$ and $\mathbb{C}_{7}=\{0000\}$ are employed.

transition probabilities are generalized, allow their application to any IrUEC trellis and to any source probability distribution $P(x)$.

Similar to the regular UEC trellis encoder, the proposed IrTrellis encoder represents each bit $y_{j}$ in the vector $\mathbf{y}$ by a codeword $\mathbf{z}_{j}$ comprising $n_{j}$ bits. This is selected from the corresponding set of $r_{j} / 2$ codewords $\mathbb{C}_{j}=\left\{\mathbf{c}_{1}^{j}, \mathbf{c}_{2}^{j}, \ldots, \mathbf{c}_{r_{j} / 2-1}^{j}\right.$, $\left.\mathbf{c}_{r_{j} / 2}^{j}\right\}$ or from the complementary set $\overline{\mathbb{C}}_{j}=\left\{\overline{\mathbf{c}_{1}^{j}}, \overline{\mathbf{c}_{2}^{j}}, \ldots\right.$, $\left.\overline{\mathbf{c}_{r_{j} / 2-1}^{j}}, \overline{\mathbf{c}_{r_{j} / 2}^{j}}\right\}$, which is achieved according to

$$
\mathbf{z}_{j}=\left\{\begin{array}{ll}
\overline{\mathbf{c}_{\left\lceil m_{j-1} / 2\right\rceil}^{j}} & \text { if } y_{j}=\operatorname{odd}\left(m_{j-1}\right) \\
\mathbf{c}_{\left\lceil m_{j-1} / 2\right\rceil}^{j} & \text { if } y_{j} \neq \operatorname{odd}\left(m_{j-1}\right)
\end{array} .\right.
$$

Finally, the selected codewords are concatenated to obtain the bit vector $\mathbf{z}=\left[z_{k}\right]_{k=1}^{b \bar{n}}$ of Fig. 1 , where $\bar{n}=\frac{1}{b} \sum_{j=1}^{b} n_{j}$ is the average codeword length. For example, the path $\mathbf{m}=$ $[1,3,5,3,2,1,1,2]$ through the irregular UEC trellis of Fig. 2 yields the encoded bit sequence $\mathbf{z}=$ [1000011111110000], which comprises $b \bar{n}=16$ bits, where we have $\bar{n}=\frac{16}{7}$.

Note that the bit vector $\mathbf{z}$ may be modeled as a specific realization of a vector $\mathbf{Z}=\left[Z_{k}\right]_{k=1}^{b \bar{n}}$ comprising $b \bar{n}$ binary RVs. Observe in Fig. 2 that each of the $b$ component trellis structures in the irregular UEC trellis of the IrTrellis encoder is designed to obey symmetry and to rely on complementary codewords. Hence, bits of the encoded bit vector $\mathbf{Z}$ have equiprobable values, where $\operatorname{Pr}\left(Z_{k}=0\right)=\operatorname{Pr}\left(Z_{k}=1\right)=0.5$, and the bit entropy obeys $H_{Z_{k}}=H\left[\operatorname{Pr}\left(Z_{k}=0\right)\right]+H\left[\operatorname{Pr}\left(Z_{k}=\right.\right.$ $1)]=1$. Owing to this, in contrast to some of the benchmarkers to be considered in Section V, the proposed IrUEC scheme of Fig. 1 does not suffer from additional capacity loss.
We assume that each of the $b$ trellis structures in the proposed 346 irregular UEC trellis is selected from a set of $S$ component 347 UEC trellis structures $\left\{\mathrm{UEC}^{S}\right\}_{s=1}^{S}$, corresponding to a set of $S 348$ component codebooks $\left\{\mathbb{C}_{s}\right\}_{s=1}^{S}$. More specifically, we assume 349 that each codebook $\mathbb{C}_{S}$ is employed for generating a particu- 350 lar fraction $\alpha_{s}$ of the bits in $\mathbf{z}$, where we have $\sum_{s=1}^{S} \alpha_{s}=1$. 351 Here, the number of bits generated using the codebook $\mathbb{C}_{s}$ is 352 given by $b \bar{n} \cdot \alpha_{s}$. We will in Section IV show that the fractions 353 $\alpha=\left\{\alpha_{S}\right\}_{s=1}^{S}$ may be designed in order to appropriately shape 354 the IrUEC EXIT function. Moreover, the IrUEC coding rate is 355 given by $R_{\mathrm{IrUEC}}=\sum_{s=1}^{S} \alpha_{s} \cdot R_{\mathrm{UEC}^{s}}$, where the corresponding 356 coding rate $R_{\mathrm{UEC}^{s}}$ of the regular $\mathrm{UEC}^{s}$ code depends on the 357 codebook $\mathbb{C}_{s}$ and is given by [10, Eq. (11)].

\section{IrURC Encoder and Modulator}

As shown in Fig. 1, the IrUEC-encoded bit sequence $\mathbf{z}$ is 360 interleaved in the block $\pi_{1}$ in order to obtain the bit vector $\mathbf{v}, \quad 361$ which is encoded by an IrURC encoder [14], [20] comprising 362 $T$ component URC codes $\left\{\mathrm{URC}^{t}\right\}_{t=1}^{T}$. Unlike our IrUEC code, 363 each component URC code URC ${ }^{t}$ of the IrURC code employs 364 a separate trellis structure. This is necessary, since the final 365 state of each component URC code has no relation to the ini- 366 tial state of the subsequent component URC code, as described 367 in Section II-B. Therefore, the interleaved IrURC-encoded bit 368 vector $\mathbf{u}$ is decomposed into $T$ sub-vectors $\left\{\mathbf{u}_{t}\right\}_{t=1}^{T}$, each having 369 a length given by $b \bar{n} \cdot \beta_{t}$, where $\beta_{t}$ represents the specific frac- 370 tion of the bits in $\mathbf{v}$ that are encoded by the component $\mathrm{URC}^{t} 371$ code, which obeys $\sum_{t=1}^{T} \beta_{t}=1$. In Section IV, we also show 372 that the fractions $\beta=\left\{\beta_{t}\right\}_{t=1}^{T}$ may be designed in order to shape 373 the IrURC EXIT function. 
In common with each of its $T$ number of component URC codes, the IrURC code has a coding rate of $R_{\text {IrURC }}=1$, regardless of the particular irregular code design. Owing to this, each of the $T$ number of binary sub-vectors $\left\{\mathbf{v}_{t}\right\}_{t=1}^{T}$ that result from IrURC encoding has the same length as the corresponding subvector $\mathbf{u}_{t}$. The set of these sub-vectors $\left\{\mathbf{v}_{t}\right\}_{t=1}^{T}$ are concatenated to obtain the bit-vector $\mathbf{v}$, which comprises $b \bar{n}$ bits.

Finally, the IrURC-encoded bit vector $\mathbf{v}$ is interleaved by $\pi_{2}$ in order to obtain the bit vector $\mathbf{w}$, which is modulated onto the uncorrelated non-dispersive Rayleigh fading channel using Gray-mapped QPSK. The overall effective throughput of the proposed scheme is given by $\eta=R_{\mathrm{IrUEC}} \cdot R_{\mathrm{IrURC}} \cdot \log _{2}(M)$, where we have $M=4$ for QPSK.

\section{IRUEC-IRURC DECODER}

In this section, we introduce the receiver of the proposed IrUEC-IrURC scheme shown in Fig. 1. In analogy with the IrURC encoder, the IrURC decoder employs $T$ number of component URC decoders $\left\{\mathrm{URC}^{t}\right\}_{t=1}^{T}$, each having a distinct independent trellis structure. By contrast, the IrUEC employs a unary decoder and a novel IrTrellis decoder relying on a single irregular trellis. In Section III-A, the demodulator and the iterative operation of the IrURC and IrUEC decoders will be discussed, while in Sections III-B and III-C we will detail the internal operation of two components of the IrUEC decoder, namely of the IrTrellis decoder and of the unary decoder, respectively.

\section{A. Demodulator and Iterative Decoding}

As shown in Fig. 1, QPSK demodulation is employed by the receiver in order to obtain the vector $\tilde{\mathbf{w}}$ of Logarithmic Likelihood Ratios (LLRs), which pertain to the bits in the vector $\mathbf{w}$. This vector is deinterleaved by $\pi_{2}^{-1}$ for the sake of obtaining the LLR vector $\tilde{\mathbf{v}}$, which is decomposed into the $T$ sub-vectors $\left\{\tilde{\mathbf{v}}_{t}\right\}_{t=1}^{T}$ that have the same lengths as the corresponding sub-vectors of $\left\{\mathbf{v}_{t}\right\}_{t=1}^{T}$. Here, we assume that a small amount of side information is used for reliably conveying the lengths of all vectors in the IrUEC-IrURC transmitter to the receiver. The sub-vectors $\left\{\tilde{\mathbf{v}}_{t}\right\}_{t=1}^{T}$ are then input to the corresponding component URC decoders $\left\{\mathrm{URC}^{t}\right\}_{t=1}^{T}$ of the IrURC decoder.

Following this, iterative exchanges of the vectors of extrinsic LLRs [21] commences between the Soft-Input Soft-Output (SISO) IrUEC and IrURC decoders. In Fig. 1, the notation $\tilde{\mathbf{u}}$ and $\tilde{\mathbf{z}}$ represent vectors of LLRs pertaining to the bit vectors $\mathbf{u}$ and $\mathbf{z}$, which are related to the inner IrURC decoder and the outer IrUEC decoder, respectively. Additionally, a subscript of this notation denotes the dedicated role of the LLRs, with $a$, $e$ and $p$ indicating a priori, extrinsic and a posteriori LLRs, respectively.

At the beginning of iterative decoding, the a priori LLR vector $\tilde{\mathbf{u}}^{\mathrm{a}}$ is initialised with a vector of zeros, having the same length as the corresponding bit vector $\mathbf{u}$. As shown in the IrURC decoder of Fig. 1, the vector $\tilde{\mathbf{u}}^{\mathrm{a}}$ is decomposed into the $T$ sub-vectors $\left\{\tilde{\mathbf{u}}_{t}^{\mathrm{a}}\right\}_{t=1}^{T}$, which have the same lengths as the corresponding sub-vectors of $\left\{\mathbf{u}_{t}\right\}_{t=1}^{T}$. Together with $\left\{\tilde{\mathbf{v}}_{t}^{\mathrm{a}}\right\}_{t=1}^{T}$, the sub-vectors $\left\{\tilde{\mathbf{u}}_{t}^{\mathrm{a}}\right\}_{t=1}^{T}$ are fed to the corresponding URC decoder 429 $\mathrm{URC}^{t}$, which then outputs the resulting extrinsic LLR vectors 430 $\left\{\tilde{\mathbf{u}}_{t}^{\mathrm{e}}\right\}_{t=1}^{T}$ by employing the logarithmic Bahl-Cocke-Jelinek- 431 Raviv (BCJR) algorithm [22]. These vectors are combined for 432 forming the extrinsic LLR vector $\tilde{\mathbf{u}}^{\mathrm{e}}$ that pertains to the vec- 433 tor $\mathbf{u}$, which is sequentially deinterleaved by the block $\pi_{1}^{-1}$ in 434 order to obtain the a priori LLR vector $\tilde{\mathbf{z}}^{\mathrm{a}}$ that pertains to the 435 bit vector $\mathbf{z}$. Similarly, the IrTrellis decoder is provided with 436 the a priori LLR vector $\tilde{\mathbf{z}}^{\mathrm{a}}$ and generates the vector of extrinsic 437 LLRs $\tilde{\mathbf{z}}^{\mathrm{e}}$, which are interleaved in the block $\pi_{1}$ to obtain the $a 438$ priori LLR vector $\tilde{\mathbf{u}}^{\mathrm{a}}$ that is provided for the next iteration of 439 the IrURC decoder.

\section{B. IrTrellis Decoder}

As discussed in Section II, our IrUEC code employs a novel 442 bit-based irregular trellis, while the IrURC code employs a 443 selection of independent trellises. The novel IrTrellis decoder 444 within the IrUEC decoder applies the BCJR algorithm to the 445 irregular trellis. The synchronization between the novel irreg- 446 ular trellis and the unary codewords is exploited during the 447 BCJR algorithm's $\gamma_{t}$ calculation of [22, (9)]. This employs 448 the conditional transition probability $\operatorname{Pr}\left(M_{j}=m_{j} \mid M_{j-1}=449\right.$ $m_{j-1}$ ), where we have

$$
P\left(m_{j} \mid m_{j-1}\right)=\frac{P\left(m_{j}, m_{j-1}\right)}{\sum_{\check{m}=1}^{r_{j}} P\left(\check{m}, m_{j-1}\right)}
$$

and $P\left(m_{j}, m_{j-1}\right)$ is given in (5).

Note that the IrUEC decoder will have an EXIT function 452 [23] that reaches the $(1,1)$ point of perfect convergence to an 453 infinitesimally low Symbol Error Ratio (SER), provided that all 454 component codebooks in the set $\left\{\mathbb{C}_{s}\right\}_{s=1}^{S}$ have a free distance of 455 at least 2 [24], as characterised in Section IV. Since the combi- 456 nation of the IrURC decoder and demodulator will also have an 457 EXIT curve that reaches the $(1,1)$ point in the top right corner 458 of the EXIT chart, iterative decoding convergence towards the 459 Maximum Likelihood (ML) performance is facilitated [25]. At 460 this point, the IrTrellis decoder may invoke the BCJR algorithm 461 for generating the vector of a posteriori LLRs $\tilde{\mathbf{y}}^{\mathrm{p}}$ that pertain to 462 the corresponding bits in the vector $\mathbf{y}$.

\section{Unary Decoder}

As described in [10], the unary decoder of Fig. 1 sorts the 465 values in the LLR vector $\tilde{\mathbf{y}}^{\mathrm{p}}$ in order to identify the $a$ number of 466 bits in the vector $\mathbf{y}$ that are most likely to have values of zero. 467 A hard decision vector $\hat{\mathbf{y}}$ is then obtained by setting the value of 468 these bits to zero and the value of all other bits to one. Finally, 469 the bit vector $\hat{\mathbf{y}}$ can be unary decoded in order to obtain the 470 symbol vector $\hat{\mathbf{x}}$ of Fig. 1, which is guaranteed to comprise $a \quad 471$ number of symbols.

\section{Algorithm for the Parametrization of the} IRUEC-IRURC SCHEME

The performance of the IrUEC-IrURC scheme depends on 475 how well it is parametrized. A good parametrization is one that 476 


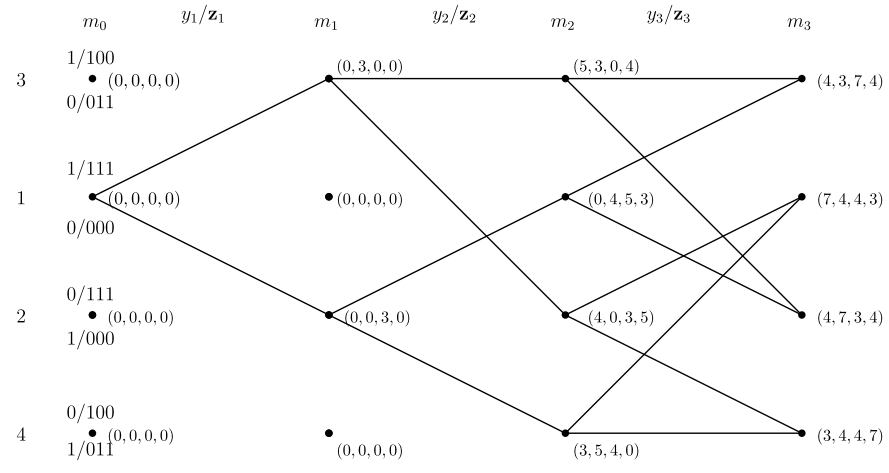

Fig. 3. The legitimate paths through the first three stages in UEC trellis having the codewords $\mathbb{C}=\{000,011\}$.

results in a narrow but still open EXIT chart tunnel, although achieving this requires a high degree of design freedom, when shaping the IrUEC and IrURC EXIT functions. Therefore, we begin in Section IV-A by characterising the free distance property of the UEC codes and selecting a set of UEC component codes having a wide variety of different inverted EXIT function shapes. This maximises the degree of freedom that is afforded, when matching the IrUEC EXIT function to that of the IrURC code. In Section IV-B, we propose a novel extension to the double-sided EXIT chart matching algorithm of [14], which we employ for jointly matching the EXIT functions of the IrUEC and the IrURC codes. However, in contrast to the algorithm of [14], which does not allow a particular coding rate to be targeted for the IrUEC-IrURC scheme, our algorithm designs both the fractions $\alpha$ and $\beta$ to achieve a particular target coding rate. In Section V, this will be exploited to facilitate a fair comparison with benchmarkers having particular coding rates.

\section{A. Design of UEC Component Codes}

Since an $r$-state $n$-bit UEC code is parametrized by a codebook set $\mathbb{C}$ comprising $r / 2$ number of codewords each having $n$ bits, there are a total of $2^{n \cdot r / 2}$ number of candidates for $\mathbb{C}$. It is neither possible nor necessary to employ all these $2^{n \cdot r / 2}$ codebooks as the component codes in our IrUEC code, because some of the codebooks will have identical or similar inverted EXIT function shapes, offering no additional degree of freedom, when performing EXIT chart matching. Therefore, it is desirable to eliminate these candidate codebooks.

The generalised UEC trellis structure associated with the codebook $\mathbb{C}=\left\{\mathbf{c}_{1}, \mathbf{c}_{2}, \ldots, \mathbf{c}_{r / 2-1}, \mathbf{c}_{r / 2}\right\}$ is depicted in [10, Fig. 3(a)]. Note that the upper half and the lower half of the trellis is symmetrical in terms of the output codewords $z_{j}$ generated in response to a given input bit value $y_{j}$, as shown in (6). More specifically, for the states in the upper half of the trellis, the output codewords $z_{j}$ are selected from the codebook $\mathbb{C}$ when $y_{j}=0$, while the codewords from its complementary codebook $\overline{\mathbb{C}}=\left\{\overline{\mathbf{c}_{1}}, \overline{\mathbf{c}_{2}}, \ldots, \overline{\mathbf{c}_{r / 2-1}}, \overline{\mathbf{c}_{r / 2}}\right\}$ are selected when $y_{j}=1$. For the states in the lower half of the trellis, the output codewords $z_{j}$ are selected from the codebook $\mathbb{C}$ when $y_{j}=1$ and from the complementary codebook $\overline{\mathbb{C}}$ when $y_{j}=0$. Intuitively, if any particular subset of the $n$ bits at the same positions within each codeword of $\mathbb{C}$ are inverted, this would not change the distance properties of the output bit vector $\mathbf{z}$, hence resulting in an identical inverted EXIT function. For example, inverting 519 the first bit of each codeword in the codebook $\mathbb{C}_{0}=\{00,01\} 520$ will give a new codebook $\mathbb{C}_{1}=\{10,11\}$ having an identical 521 EXIT function. Likewise, inverting both bits of the codewords 522 in $\mathbb{C}_{0}$ will give $\mathbb{C}_{2}=\{11,10\}$, which also has an identical EXIT 523 function. Similarly, swapping any pair of the $n$ bits at the same 524 positions between each pair of codewords will not affect the 525 distance properties or the shape of the inverted EXIT function 526 either. For example, swapping the two bits in the codebook $\mathbb{C}_{0} 527$ results in a new codebook $\mathbb{C}_{3}=\{00,10\}$, having an identical 528 inverted UEC EXIT function shape. Therefore, each of these 529 four codebooks, $\mathbb{C}_{0}, \mathbb{C}_{1}, \mathbb{C}_{2}$ and $\mathbb{C}_{3}$, as well as their conversions 530 created by bit-inversion and swapping, have identical inverted 531 EXIT functions. Consequently, all but one of these codebooks 532 can be eliminated as candidates for the sake of reducing the 533 complexity of EXIT chart matching.

The number of candidate UEC codebooks may be further 535 reduced by characterising their free distance properties. Since 536 no analytic method has been developed for calculating the free 537 distance $d_{f}$ of a UEC code, we propose a heuristic method 538 for obtaining an approximate measure of $d_{f}$. The free dis- 539 tance represents the minimum distance between any pair of 540 encoded bit vectors produced by different paths through the 541 trellis. The total number of possible pairings of paths emerg- 542 ing from a particular state in a UEC trellis of length $b$ is given 543 by $2^{b-1}\left(2^{b}-1\right)$, which grows exponentially. However, consid- 544 ering the symmetry of a regular UEC trellis, it is possible to 545 use a step-by-step directed search for determining the free dis- 546 tance, rather than using a brute force exhaustive search. Note 547 that in the regular UEC trellis as generalised in [10, Fig. 3(a)], 548 a bit vector $\mathbf{y}=\left[y_{j}\right]_{j=1}^{b}$ identifies a unique path $\mathbf{m}=\left[m_{j}\right]_{j=0}^{b} 549$ that emerges from state 1 and terminates at either state 1550 or 2, hence accordingly identifying a corresponding output 551 bit sequence $\mathbf{z}=\left[z_{k}\right]_{k=1}^{b \bar{n}}$. By exploiting this observation, the 552 free distance $d_{f}$ can be obtained by computing the Hamming 553 Distance(HD) between each pair of paths and then selecting the 554 pair having the minimum HD, whenever two paths merge at a 555 particular state in the trellis. 556

When the bit sequence length considered satisfies $b>557$ $r / 2$, the paths form complete trellis stages, as exemplified 558 in Fig. 3. Therefore, in order to reduce the search complex- 559 ity, we consider all permutations of the $b$-bit unary-encoded 560 vector $\mathbf{y}$ bit-by-bit, considering all paths that emerge from 561 state $m_{0}=1$ and terminate at each particular state $m_{b}=562$ $1,2, \ldots, r$, on a step-by-step basis. For a pair of states 563 $m_{j}, m_{j}^{\prime} \in\{1,2,3, \ldots, r\}$, we define $d_{m_{j}, m_{j}^{\prime}}^{j}$ as the minimum 564 HD between the set of all paths that terminate at state $m_{j} 565$ and the set that ends at state $m_{j}^{\prime}$, given the input bit sequence 566 $\left[y_{1}, y_{2}, \ldots, y_{j}\right]$, where $j \in\{0,1, \ldots, b\}$. Each state $m_{j}$ is 567 labelled as $\left(d_{m_{j}, 1}^{j}, d_{m_{j}, 2}^{j}, d_{m_{j}, 3}^{j}, \ldots, d_{m_{j}, r}^{j}\right)$, where we have 568 $d_{m_{j}, m_{j}^{\prime}}^{j}=d_{m_{j}^{\prime}, m_{j}}^{j}$. For each state $m_{0} \in\{1,2,3, \ldots, r\}$, the min- 569 imum HDs are initialized to 0s. Therefore, the distance $d_{m_{j}, m_{j}^{\prime}}^{j}$ can be calculated by

$$
d_{m_{j}, m_{j}^{\prime}}^{j}=\min _{m_{j-1}, m_{j-1}^{\prime}}\left[d_{m_{j-1}, m_{j-1}^{\prime}}^{j-1}+h\left(\mathbf{z}_{m_{j-1}, m_{j}}, \mathbf{z}_{m_{j-1}^{\prime}, m_{j}^{\prime}}\right)\right] .
$$


Here, $\mathbf{z}_{m_{j-1}, m_{j}}$ is the codeword in the set $\mathbb{C}$ or in the complementary set $\overline{\mathbb{C}}$ that is generated by the transition from state $m_{j-1}$ to state $m_{j}$, while the function $h(\cdot, \cdot)$ denotes the HD between the two operands. Owing to this, our method conceived for determining the free distance of a UEC code has a complexity order of $O[b \cdot r(r-1)]$, where $r$ is the number of states in the trellis and $b$ is the length of the bit vector $\mathbf{y}$ considered. Let $\mathbb{Y}_{b_{1}}$ be the bit sequence set associated with the set of all paths $\mathbb{M}_{b_{1}}$ having a length of $b_{1}$, while $\mathbb{Y}_{b_{2}}$ is the bit sequence set associated with the path set $\mathbb{M}_{b_{2}}$ having a length of $b_{2}$. Therefore, all sequences in $\mathbb{Y}_{b_{1}}$ are prefix of sequences in $\mathbb{Y}_{b_{2}}$, when we have $b_{1}<b_{2}$. For example, when $b_{1}=2$ and $b_{2}=3$, the bit sequence $\mathbf{y}_{2}=\{111011\}$ is a prefix of the bit sequence $\mathbf{y}_{3}=\{111011111\}$, where $\mathbf{y}_{2}$ is associated with the path vector $\mathbf{m}_{2}=\{1,3,2\}$ and $\mathbf{y}_{2}$ is associated with the path vector $\mathbf{m}_{2}=\{1,3,2,1\}$, respectively. Note that according to [26, Lemma 1], the minimum $\mathrm{HD} d_{f}\left(\mathbb{Y}_{b_{1}}\right)$ among all bit sequences in $\mathbb{Y}_{b_{1}}$ is an upper bound on the minimum HD $d_{f}\left(\mathbb{Y}_{b_{2}}\right)$ of $\mathbb{Y}_{b_{2}}$, when we have $b_{1}<b_{2}$. Owing to this, the approximate free distance $d_{f}$ calculated using our method converges to the true free distance, as the lengths of the paths considered are extended towards infinity. In our experiments, we considered bit vector lengths of up to $b=10 r$. In all cases, we found that the free distance has converged before that point, regardless of how the UEC code is parametrised, owing to the common features of all UEC codes described in Section II-B."

For example, Fig. 3 shows all of the legitimate paths through an $r=4$-state trellis employing the codebook $\mathbb{C}=$ $\{000,011\}$ that may be caused by the first three bits in a bit vector $\mathbf{y}=\left\{y_{j}\right\}_{j=1}^{b}$, having a length $b>3$. Particularly, the minimum HD $d_{2,3}^{1}$ between states $m_{1}=2$ and $m_{1}^{\prime}=3$ is given by $d_{2,3}^{1}=d_{1,1}^{0}+h(111,000)=3$. Since there are no legitimate paths leading to the states $m_{1}=1$ or $m_{1}=$ 4 , we do not update the associated distances, as shown in Fig. 3. Similarly, we have $d_{1,2}^{2}=d_{2,3}^{1}+h(111,011)=4$, and $d_{1,2}^{3}=\min \left(d_{1,2}^{2}+h(000,111), d_{1,4}^{2}+h(000,100), d_{2,3}^{2}+\right.$ $\left.h(111,011), d_{3,4}^{2}+h(011,100)\right)=4$. Once the forward recursion has considered a sufficient number of trellis stages for $\min \left(d_{1,1}^{j}, d_{1,2}^{j}, d_{2,2}^{j}\right)=\min \left(d_{1,1}^{j-1}, d_{1,2}^{j-1}, d_{2,2}^{j-1}\right)$, then the approximate free distance becomes $d_{f}=\min \left(d_{1,1}^{j}, d_{1,2}^{j}, d_{2,2}^{j}\right)$.

Our set of candidate component UEC codes was further reduced by considering their free distances. More specifically, in order to achieve a wide variety of EXIT function shapes, we retained only UEC codebooks having the maximal or minimal free distances for each combination of $n \in\{2,3,4\}$ and $r \in\{2,4\}$, where a free distance of 3 is the minimal value that facilitates convergence to the $(1,1)$ point $[24]$ and avoids an error floor. We drew the EXIT functions for all remaining candidate component UEC codes and selected the five codebooks offering the largest variety of EXIT function shapes, as listed in Table II. Our experiments revealed that only insignificant EXIT function shape variations are obtained, when considering more than $r=4$ states. Without loss of generality, our irregular trellis example of Fig. 2 is constructed by concatenating the five UEC codebooks of Table II. In the following simulations, we will consider irregular trellises that are constructed using these
TABLE II

AFter INVERTING AND SWAPPING, We SELECT the IRUEC COMPONENT UEC CODEBOoKS $\left\{\mathbb{C}_{s}\right\}_{s=1}^{5}$ WITH $n$ BITS AND $r$ STATES Both up to 4 . All the Codebooks ARe in the Format $\left(\mathbb{C}_{s}, d_{f}\right)$, Where $d_{f}$ IS The Approximate Free Distance

\begin{tabular}{|l|l|l|}
\hline$n$ & $r=2$ & $r=4$ \\
\hline 2 & & $(\{00,01\}, 3)$ \\
\hline 3 & $(\{000\}, 3)$ & $(\{000,011\}, 4)$ \\
\hline 4 & $(\{0000\}, 4)$ & $(\{0000,0111\}, 5)$ \\
\hline
\end{tabular}

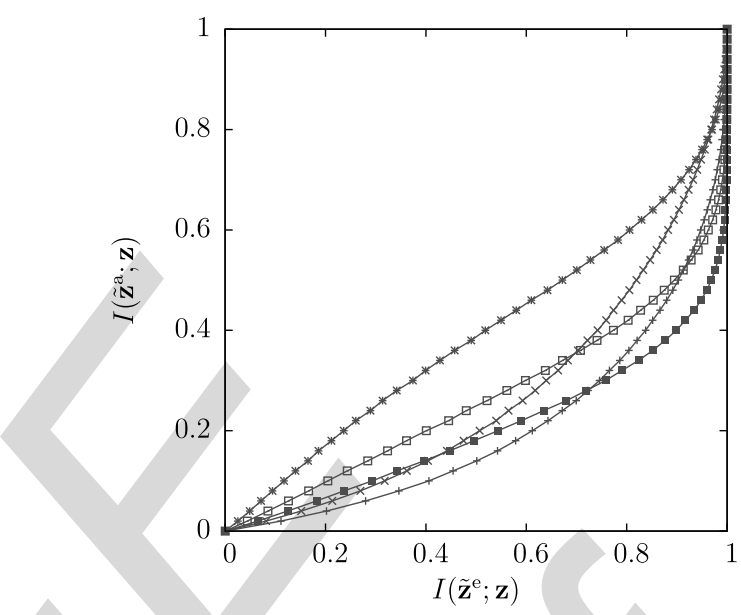

Fig. 4. Inverted EXIT functions for the $S=5$ component UEC codes $\left\{\mathrm{UEC}^{S}\right\}_{s=1}^{5}$ of Table II, when extended to $r=10$ states codebooks, and when the symbol values obey a zeta probability distribution having the parameter value $p_{1}=0.797$.

codebooks. However, the number of states $r$ employed by our 628 five UEC component codes can be optionally and independently 629 increased in the receiver, in order to facilitate nearer-to-capacity 630 operation at the cost of an increased decoding complexity [10]. 631 This is achieved by repeating the last element in the code- 632 book. For example, while the transmitter may use the codebook 633 $\mathbb{C}=\{00,01\}$, the receiver may extend this to the $r=10$-state 634 codebook $\mathbb{C}=\{00,01,01,01,01\}$. Fig. 4 plots the inverted 635 EXIT functions of the component UEC codes $\left\{\mathrm{UEC}^{s}\right\}_{s=1}^{5}, 636$ when extended to $r=10$ states. Note that, similar to the IrURC 637 EXIT function, the composite IrUEC EXIT function $f_{\text {IrUEC }}$ is 638 given as a weighted average of the component EXIT functions 639 $\left\{f_{\mathrm{UEC}^{s}}\right\}_{s=1}^{5}$, where we have

$$
f_{\mathrm{IrUEC}}=\sum_{s=1}^{5} \alpha_{s} \cdot f_{\mathrm{UEC}^{s}} .
$$

\section{B. Double-Sided EXIT Chart Matching Algorithm}

The sixth column of Table III provides the specific $E_{\mathrm{b}} / N_{0} 642$ values, where the DCMC capacity becomes equal to the 643 throughput $\eta$ of each scheme considered. These $E_{\mathrm{b}} / N_{0}$ values 644 represent the capacity bound, above which it is theoretically 645 possible to achieve reliable communication. Note that the 646 capacity bound is a function of the overall effective throughput 647 $\eta$ of the proposed IrUEC scheme, as described in Section II- 648 C. In turn, the overall effective throughput $\eta$ depends on the 649 
TABLE III

Characteristics of The VArious Schemes Considered, INCLuding Outer Coding RATE $R_{\mathrm{O}}$, InNer Coding RATE $R_{\mathrm{I}}$ AND EFFECTIVE THRoughrut $\eta$. $E_{\mathrm{B}} / N_{0}$ BOUNDS ARE GIVEN FOR THE CASE OF GRAY-CODED QPSK TRANSMISSION OVER

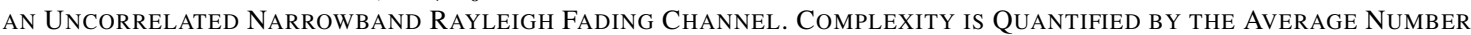
of ACS Operations INCURREd PER DECOdING ITERATION AND PER Bit IN THE Vector $\mathbf{z}$

\begin{tabular}{|c|c|c|c|c|c|c|c|c|}
\hline 1 & 2 & 3 & 4 & 5 & 6 & 7 & 8 & 9 \\
\hline Scheme & Codebooks & $R_{\mathrm{o}}$ & $R_{\mathrm{i}}$ & $\eta$ & $\begin{array}{c}E_{\mathrm{b}} / N_{0}[\mathrm{~dB}] \\
\text { capacity bound }\end{array}$ & $\begin{array}{c}E_{\mathrm{b}} / N_{0}[\mathrm{~dB}] \\
\text { area bound }\end{array}$ & $\begin{array}{l}E_{\mathrm{b}} / N_{\mathrm{o}}[\mathrm{dB}] \\
\text { tunnel bound }\end{array}$ & Complexity \\
\hline IrUEC-IrURC & $\multirow{3}{*}{\mathrm{UEC}^{s}}_{s=1}^{5}$ & \multirow{4}{*}{0.254} & \multirow{4}{*}{1} & \multirow{7}{*}{0.508} & \multirow{7}{*}{-0.05} & 0.21 & $\overline{0.3}$ & 258 \\
\hline IrUEC(med)-IrURC & & & & & & 0.30 & 0.6 & 192 \\
\hline IrUEC(low)-IrURC & & & & & & 1.14 & 1.2 & 157 \\
\hline UEC-IrURC & $\{000,011\}$ & & & & & 0.49 & 1.7 & 120 \\
\hline \multirow{2}{*}{ EG-IrCC-IrURC } & $\left\{\mathbf{C C}_{\mathrm{sys}}^{\mathrm{s}}\right\}_{\mathrm{s}=1}^{13}$ & 0.667 & 0.576 & & & 1.72 & 2.0 & 341 \\
\hline & $\left\{\mathbf{C} \mathbf{C}_{\mathrm{ns}}^{s}\right\}_{s=1}^{11}$ & \multirow{2}{*}{0.254} & \multirow{2}{*}{1} & & & 1.02 & 1.1 & 146 \\
\hline EG-CC-IrURC & $([4,7,7], 6,6)$ & & & & & 1.00 & 2.2 & 132 \\
\hline
\end{tabular}

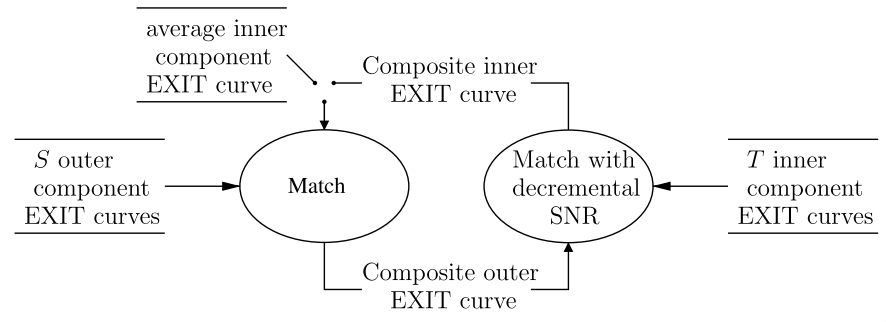

Fig. 5. Data-flow diagram of the proposed double-sided EXIT chart matching algorithm.

IrUEC coding rate $R_{\text {IrUEC }}$, which depends on the entropy of the zeta distribution $H_{X}$, as described in Section II-A. In order to facilitate the creation of an open EXIT chart tunnel, it is necessary, but not sufficient, for the area $A_{\mathrm{o}}$ beneath the inverted outer EXIT function to exceed the area $A_{\mathrm{i}}$ beneath the inner EXIT function [17]. Therefore, the area bound provides the $E_{\mathrm{b}} / N_{0}$ values where we have $A_{\mathrm{o}}=A_{\mathrm{i}}$, which would theoretically allow the creation of an open EXIT chart tunnel [27], if the outer and inner EXIT functions were shaped to match each other. Here, $A_{0}$ and $A_{\mathrm{i}}$ are the areas beneath the outer and inner EXIT functions, respectively. Depending on how well the EXIT functions match each other, a narrow but open EXIT chart tunnel can only be created at a specific $E_{\mathrm{b}} / N_{0}$ value, which we refer to as the tunnel bound. Based on these observations, the $E_{\mathrm{b}} / N_{0}$ difference between the capacity bound and the area bound quantifies the capacity loss that is mitigated by JSCC, while the difference between the area bound and the tunnel bound quantifies the capacity loss that is mitigated by irregular coding [28]. Based on this observation, our doublesided EXIT chart matching algorithm may be iteratively applied in order to match a pair of composite outer and inner EXIT functions, which are formed as a combination of $S$ component UEC EXIT functions and $T$ constituent URC EXIT functions, where the latter depend on the $E_{\mathrm{b}} / N_{0}$ value of the channel. In this way, a narrow but open EXIT chart tunnel between the inverted IrUEC EXIT function and the inner IrURC EXIT function may be created at $E_{\mathrm{b}} / N_{0}$ values that approach the capacity and area bounds, hence avoiding capacity loss and facilitating near-capacity operation.

As depicted in the data-flow diagram of Fig. 5, the algorithm commences by selecting the fractions $\alpha$, in order to yield an IrUEC code design having a particular coding rate $R_{\text {IrUEC }}$ and a composite IrUEC EXIT function that is shaped to match the 682 average of $T$ URC EXIT functions that correspond to a partic- 683 ular $E_{\mathrm{b}} / N_{0}$ value. The technique of [14] may be employed for 684 selecting the fractions $\beta$, in order to yield a composite IrURC 685 EXIT function that is shaped to match that of the IrUEC code. 686 Following this, the algorithm alternates between the matching 687 of the composite IrUEC EXIT function to the composite IrURC 688 EXIT function and vice versa, as shown in Fig. 5. In order 689 to facilitate near-capacity operation, we use a $0.1 \mathrm{~dB} E_{\mathrm{b}} / N_{0} 690$ decrement per iteration for the component URC EXIT func- 691 tions, when designing the fractions $\beta$ for the IrURC code, until 692 we find the lowest $E_{\mathrm{b}} / N_{0}$ value that achieves a marginally open 693 EXIT tunnel. Note that the double-sided EXIT chart matching 694 algorithm allows the design of an IrUEC code having a spe- 695 cific coding rate $R_{\text {IrUEC }}$. This enables us to design the IrUEC 696 code to have a coding rate of $R_{\mathrm{IrUEC}}=0.254$, which provides 697 a fair performance comparison with the regular UEC-IrURC 698 scheme of [10] and with other benchmarkers, as detailed in 699 Section V. More specifically, this results in the same overall 700 effective throughput of $\eta=R_{\mathrm{IrUEC}} \cdot R_{\mathrm{IrURC}} \cdot \log _{2}(M)=0.508701$ $\mathrm{bit} / \mathrm{s} / \mathrm{Hz}$, as listed in Table III.

For the IrURC encoder, we employ the $T=10$-component 703 URC codes $\left\{\mathrm{URC}^{t}\right\}_{t=1}^{10}$ of [20], [29]. After running the double- 704 sided EXIT chart matching algorithm of Fig. 5 until the $E_{\mathrm{b}} / N_{0} \quad 705$ value cannot be reduced any further without closing the EXIT 706 chart tunnel, the composite EXIT functions of the IrUEC and 707 IrURC schemes are obtained, as depicted in Fig. 6(a). Here, the 708 $E_{\mathrm{b}} / N_{0}$ value is $0.3 \mathrm{~dB}$, which is $0.35 \mathrm{~dB}$ away from the DCMC 709 capacity bound of $-0.05 \mathrm{~dB}$ and was found to be the lowest one 710 that creates an open EXIT chart tunnel. More specifically, the 711 fractions of the bit vector $\mathbf{z}$ that are generated by the constituent 712 UEC codes $\left\{\mathrm{UEC}^{s}\right\}_{s=1}^{5}$ of the IrUEC encoder are $\alpha=\left[\begin{array}{lll}0 & 0.7240 & 713\end{array}\right.$ $0.092400 .1836]$, respectively. Similarly, the fractions of the bit 714 vector $\mathbf{u}$ that encoded by the constituent URC codes $\left\{\mathrm{URC}^{t}\right\}_{t=1}^{10} 715$ of the IrURC encoder are $\beta=\left[\begin{array}{llllllllll}0.1767 & 0 & 0.8233 & 0 & 0 & 0 & 0 & 0 & 0 & 0\end{array}\right], 716$ respectively.

\section{BENCHMARKERS AND SIMULATIONS}

In this section, we compare the SER performance of the 719 proposed IrUEC-IrURC scheme of Fig. 1 to that of various 720 SSCC and JSCC benchmarkers. As mentioned in Section IV, 721 the proposed IrUEC-IrURC scheme and all benchmarkers are 722 designed to have the same effective overall throughput of 723 


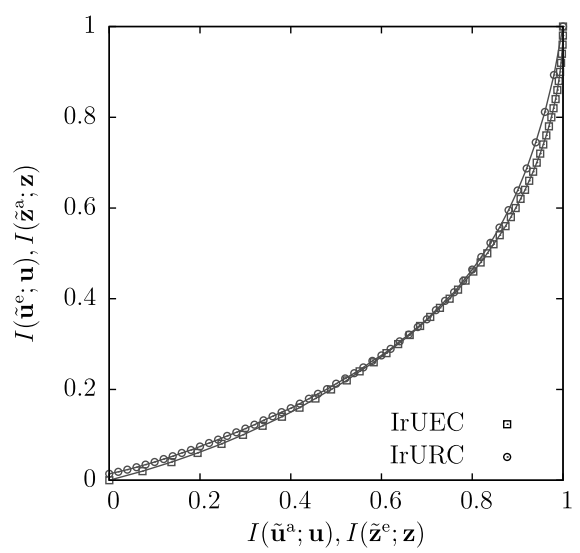

(a)

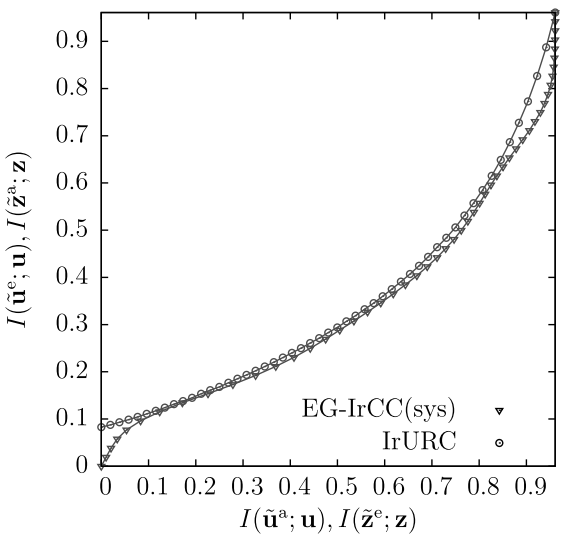

(b)

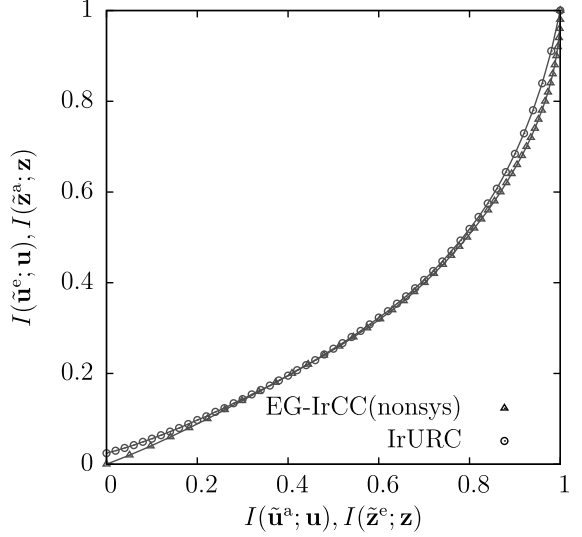

(c)

Fig. 6. Composite EXIT functions of (a) the IrUEC decoder employing $S=5$ component UEC codes $\left\{\text { UEC }^{s}\right\}_{s=1}^{5}$, (b) the EG-IrCC decoder employing the $S=13$ component recursive systematic CC codes $\left\{\mathbf{C C}_{\mathrm{sys}}^{s}\right\}_{s=1}^{13}$ and (c) the EG-IrCC scheme employing the $S=11$ component non-systematic CC codes $\left\{\mathbf{C C}_{\mathrm{ns}}^{s}\right\}_{s=1}^{11}$, and the IrURC scheme employing the $T=10$ component URC codes $\left\{\mathrm{URC}^{t}\right\}_{t=1}^{10}$, when conveying symbols obey a zeta distribution having the parameter $p_{1}=0.797$, and communicating over a QPSK-modulated uncorrelated narrowband Rayleigh fading channel. The EXIT chart tunnel is marginally open when $E_{\mathrm{b}} / N_{0}=0.3$, 2.0 and $1.1 \mathrm{~dB}$, respectively.

EG-IrCC Encoder
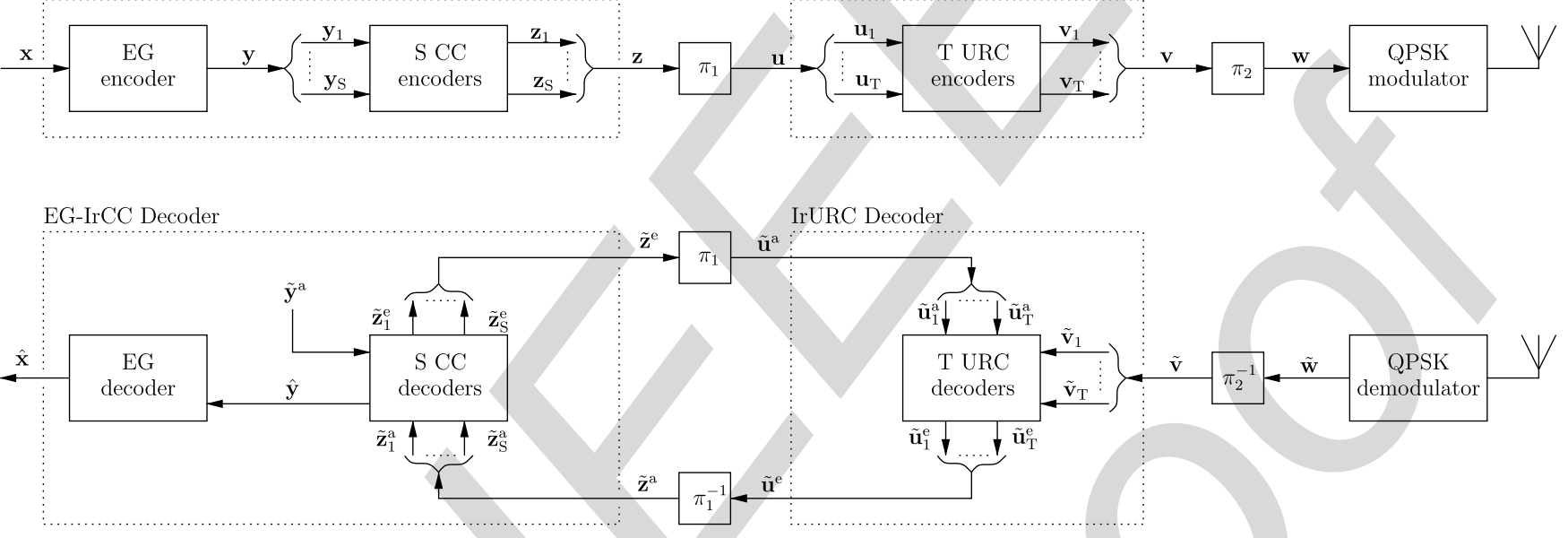

Fig. 7. Schematic of the EG-IrCC-IrURC benchmarker, in which an EG-IrCC code is serially concatenated with IrURC code and Gray-coded QPSK modulation schemes. Here, $\pi_{1}$ and $\pi_{2}$ represent interleavers, while $\pi_{1}^{-1}$ and $\pi_{2}^{-1}$ represent the corresponding deinterleavers.

$\eta=0.508 \mathrm{bit} / \mathrm{s} / \mathrm{Hz}$, for the sake of fair comparison. A pair of benchmarkers are constituted by the UEC-IrURC and EGCC-IrURC schemes of our previous work [10]. Furthermore, a new benchmarker is created by replacing the unary encoder and the IrTrellis encoder in the transmitter of Fig. 1 with an EG encoder and an IrCC encoder, respectively. This results in the SSCC benchmarker of Fig. 7, which we refer to as the EGIrCC-IrURC scheme. Table I shows the first ten codewords of the EG code, which are used for encoding the symbol vector $\mathbf{x}$.

As in the IrUEC-IrURC scheme, the bit vector $\mathbf{y}$ output by the EG encoder may be modeled as a realization of vector $\mathbf{Y}=$ $\left[Y_{j}\right]_{j=1}^{b}$ having binary RVs. However, as observed in [10], these RVs do not adopt equiprobable values $\operatorname{Pr}\left(Y_{j}=0\right) \neq \operatorname{Pr}\left(Y_{j}=\right.$ $1)$, hence giving a less than unity value for the corresponding bit entropy $H_{Y_{j}}$. Similarly, the bit vector $\mathbf{z}$ of Fig. 7 may be modeled as a particular realization of a vector $\mathbf{Z}=\left[Z_{k}\right]_{k=1}^{b \bar{n}}$ comprising $b \bar{n}$ binary RVs. Each binary RV $Z_{k}$ adopts the values 0 and 1 with the probabilities $\operatorname{Pr}\left(Z_{k}=0\right)$ and $\operatorname{Pr}\left(Z_{k}=1\right)$ respectively, corresponding to a bit entropy of $H_{Z_{k}}$. In the case where the IrCC code employs systematic component codes, the 743 bits of $\mathbf{y}$ having the entropy $H_{Y_{j}}<1$ will appear in $\mathbf{z}$, resulting 744 in a bit entropy of $H_{Z_{k}}<1$. However, a bit entropy of $H_{Z_{k}}<1745$ is associated with a capacity loss, as described in [10].

Hence, for the sake of avoiding any capacity loss, it is 747 necessary to use non-systematic recursive component codes, so 748 that the bits in the resultant encoded vector $\mathbf{z}$ have equiprob- 749 able values [10]. In order to demonstrate this, we introduce 750 two versions of the EG-IrCC-IrURC benchmarker. Firstly, 751 the $N=13$ recursive systematic component CC codes [15] 752 $\left\{\mathbf{C C}_{\text {sys }}^{s}\right\}_{s=1}^{13}$ that were originally proposed for IrCC encoding 753 are adopted in the EG-IrCC-IrURC encoder, as it will be 754 described in Section V-A. Secondly, Section V-B employs the 755 $S=11$ non-systematic recursive CC codebooks $\left\{\mathbf{C C}_{\mathrm{ns}}^{S}\right\}_{s=1}^{11} 756$ proposed in [20], in order to offer an improved version of the 757 EG-IrCC benchmarker. Meanwhile, the 10 component URC 758 codebooks $\left\{\mathrm{URC}^{t}\right\}_{t=1}^{10}$ employed by the IrURC encoder in both 759 versions of the benchmarker of Fig. 7 are identical to those in 760 the IrURC encoder of Fig. 1. 


\section{A. Recursive Systematic Component CC Codes}

763

764

765

766

767

768

769

770

771

772

773

774

775

776
The recursive systematic CC codes $\left\{\mathbf{C C}_{\text {sys }}^{s}\right\}_{s=1}^{13}$ employed in [15] were designed to have coding rates of $R_{\mathrm{CC}_{\mathrm{sys}}^{s}} \in$ $\{0.1,0.15, \ldots, 0.65,0.7\}$. However, since the EG-encoded bits in the vector $\mathbf{y}$ are not equiprobable, none of the systematic bits in the bit vector $\mathbf{z}$ will be equiprobable either. As a result, the coding rate $R_{\mathrm{CC}_{\mathrm{sys}}^{s}}=\frac{H_{Y_{j}}}{n_{\mathrm{CC}_{\mathrm{sys}}^{s}} \cdot H_{Z_{k}}^{\mathrm{CC}_{\mathrm{sys}}^{s}}}$ of each systematic $\mathrm{CC}$ will be lower than the above-mentioned values. Since each $\mathrm{CC}$ code $\mathbf{C C}_{\text {sys }}^{s}$ produces a different number of systematic bits, each will have a different bit entropy $H_{Z_{k}}^{\mathrm{CC}_{\text {sys }}^{s}}$, and the EXIT function of each CC code will converge to a different point $\left(H_{Z_{k}}^{\mathbf{C C}} \mathbf{C}_{\text {sys }}^{s}, H_{Z_{k}}^{\mathbf{C C}} \mathbf{C}_{\text {sys }}^{s}\right)$ in the EXIT chart [30]. The composite IrCC EXIT function will converge to a point $\left(H_{Z_{k}}^{\mathrm{IrCC}}, H_{Z_{k}}^{\mathrm{IrCC}}\right)$, where $H_{Z_{k}}^{\mathrm{IrCC}}$ is given by a weighted average of $\left\{H_{Z_{k}}^{\mathbf{C C}_{\text {sys }}^{s}}\right\}_{s=1}^{13}$, according to

$$
H_{Z_{k}}^{\mathrm{IrCC}}=\sum_{s=1}^{13} \alpha_{s} \cdot H_{Z_{k}}^{\mathbf{C C}_{\mathrm{sys}}^{s}} .
$$

Since the vector $\mathbf{z}$ is interleaved to generate the bit vector $\mathbf{u}$ as the input of the IrURC encoder, the IrURC EXIT function will also converge to $\left(H_{Z_{k}}^{\mathrm{IrCC}}, H_{Z_{k}}^{\mathrm{IrCC}}\right)$. However, this presents a particular challenge, when parametrizing the fractions $\alpha$ and $\beta$ of the EG-IrCC(sys)-IrURC scheme. More specifically, the fractions $\alpha$ vary as our double-sided EXIT chart matching algorithm progresses, causing the entropy $H_{Z_{k}}^{\mathrm{IrCC}}$ to vary as well. This in turn causes the IrURC EXIT function to vary, creating a cyclical dependency that cannot be readily resolved. More specifically, the fractions $\alpha$ must be selected to shape the EG-IrCC EXIT function so that it matches the IrURC EXIT function, but the IrURC EXIT function depends on the fractions $\alpha$ selected for the EG-IrCC EXIT function.

Owing to this, we design the fractions $\alpha$ and $\beta$ by assuming that the bits of $\mathbf{y}$ are equiprobable and by plotting the inverted EXIT functions for the $S=13$ recursive systematic CC codes accordingly, giving convergence to the $(1,1)$ point in Fig. 6(b). Then we invoke our double-sided EXIT matching algorithm to design the fractions $\alpha$ and $\beta$ for the $\operatorname{IrCC}$ (sys) and IrURC codes, which we apply to the EG-IrCC(sys)-IrURC scheme. For the case where the bits of the vector $\mathbf{y}$ have the non-equiprobable values that result from EG encoding, the composite EXIT functions are shown in Fig. 6(b). Here, the effective throughput is $\eta=0.508 \mathrm{bit} / \mathrm{s} / \mathrm{Hz}$ and the $E_{\mathrm{b}} / N_{0}$ value is $2.0 \mathrm{~dB}$, which is the lowest value for which an open EXIT chart tunnel can be created. This $E_{\mathrm{b}} / N_{0}$ tunnel bound is $2.05 \mathrm{~dB}$ away from the DCMC capacity bound of $-0.05 \mathrm{~dB}$, owing to the above-mentioned capacity loss. Furthermore, the EG-IrCC(sys)-IrURC scheme has an area bound of $1.72 \mathrm{~dB}$, which corresponds to a capacity loss of $1.77 \mathrm{~dB}$, relative to the capacity bound. The designed fractions for the EGIrCC scheme are $\alpha=\left[\begin{array}{ll}0.06200 .29970 .04970 .00040 .19430 \\ 0.098\end{array}\right.$ $0.09840 .128500000 .00020 .1668]$, while the fractions for the IrURC code are $\beta=\left[\begin{array}{lllllllllll}0.6548 & 0 & 0.3452 & 0 & 0 & 0 & 0 & 0 & 0 & 0\end{array}\right]$, respectively.

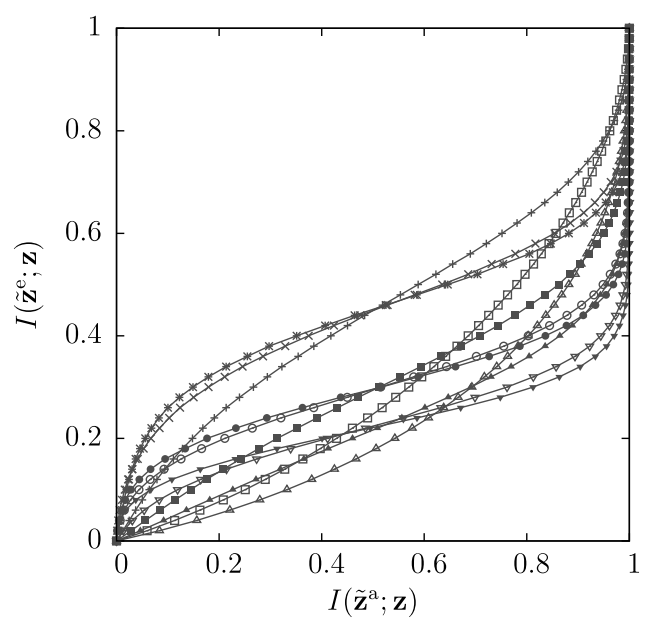

Fig. 8. Inverted EXIT functions for EG-CC code, for the case where the $S=11$ component recursive non-systematic CC codes $\left\{\mathbf{C C}_{\mathrm{ns}}^{S}\right\}_{s=1}^{11}$ are employed, and the symbol values obey a zeta probability distribution having the parameter value $p_{1}=0.797$.

\section{B. Recursive Non-Systematic Component CC Codes}

In order to avoid the capacity loss introduced by the recursive systematic $\mathrm{CC}$ codes, we advocate the recursive non-systematic $\mathrm{CC}$ codebooks $\left\{\mathbf{C C}_{\mathrm{ns}}^{s}\right\}_{s=1}^{11}$, which are described by the generator and feedback polynomials provided in [10, Table II]. More specifically, of the 12 codes presented in [10, Table II], we use all but the $r=2, n=2$ code, for the sake of avoiding an error floor. These recursive non-systematic CC codes attain the optimal distance properties [31] subject to the constraint of producing equiprobable bits $\operatorname{Pr}\left(Z_{j}=0\right)=\operatorname{Pr}\left(Z_{j}=1\right)$, which is necessary for avoiding any capacity loss. The inverted EXIT functions are plotted in Fig. 8.

For the sake of a fair comparison, we apply the doublesided EXIT chart matching algorithm of Fig. 5 again to design the EG-IrCC(nonsys)-IrURC scheme having a coding rate of $R_{\mathrm{EG}-\mathrm{IrCC}}=0.254$ and an effective throughput of $\eta=$ $0.508 \mathrm{bit} / \mathrm{s} / \mathrm{Hz}$. The composite EXIT functions of the EGIrCC(nonsys) and IrURC schemes are shown in Fig. 6(c). Here, the fractions of the EG-IrCC scheme are $\alpha=[0.810100 .0643$ 00000.1256000 ], while the fractions of the IrURC code are

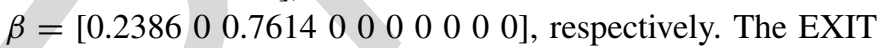
chart of Fig. 8 is provided for an $E_{\mathrm{b}} / N_{0}$ value of $1.1 \mathrm{~dB}$, which is the lowest value for which an open EXIT chart tunnel is created. As shown in Table III, this $E_{\mathrm{b}} / N_{0}$ tunnel bound is just $1.15 \mathrm{~dB}$ away from the DCMC capacity bound of $-0.05 \mathrm{~dB}$. This improvement relative to the EG-IrCC(sys)-IrURC scheme may be attributed to the non-systematic nature of the EGIrCC(nonsys)-IrURC scheme, which has reduced the capacity loss to $1.07 \mathrm{~dB}$, as quantified by considering the difference between the $E_{\mathrm{b}} / N_{0}$ area bound of $1.02 \mathrm{~dB}$ and the capacity bound.

\section{Parallel Component UEC Codes}

In order to make a comprehensive comparison, we also con- 844 sider a Parallel IrUEC-IrURC scheme. As shown in Fig. 9, 845 this scheme employs a parallel concatenation of $S$ number 846 

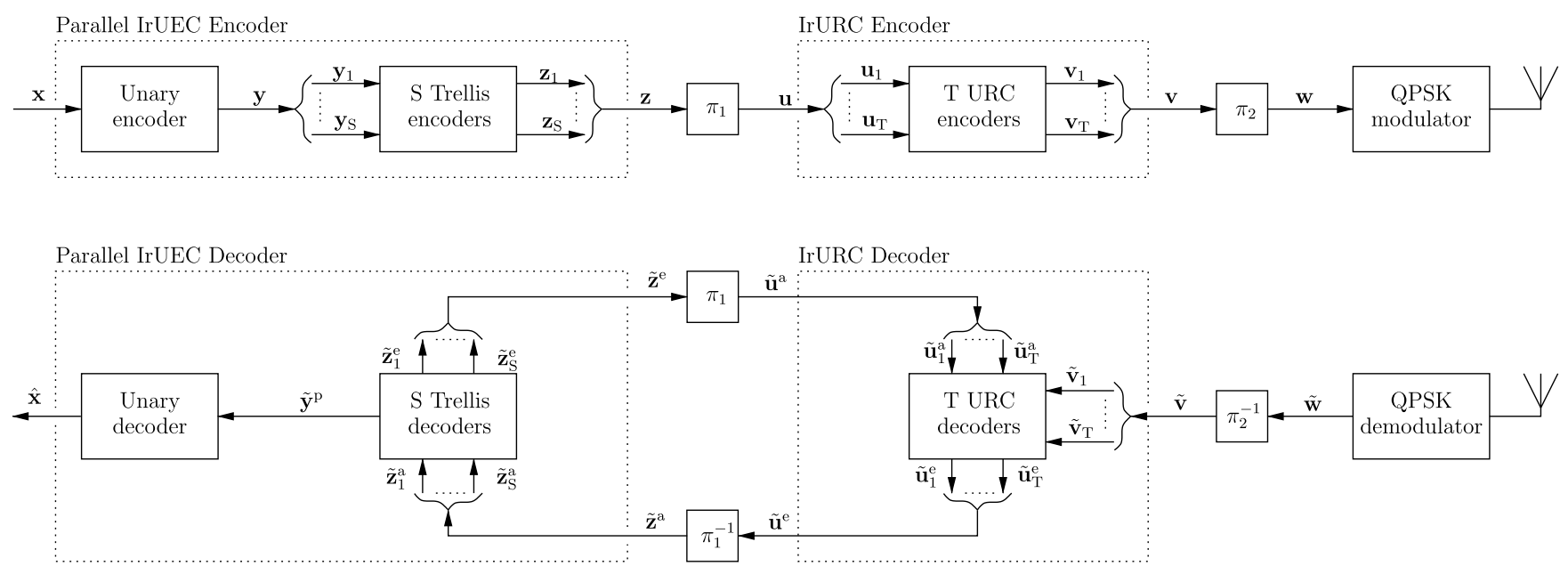

Fig. 9. Schematic of the Parallel IrUEC-IrURC benchmarker, in which a parallel IrUEC code is serially concatenated with IrURC code and Gray-coded QPSK modulation schemes. Here, $\pi_{1}$ and $\pi_{2}$ represent interleavers, while $\pi_{1}^{-1}$ and $\pi_{2}^{-1}$ represent the corresponding deinterleavers.

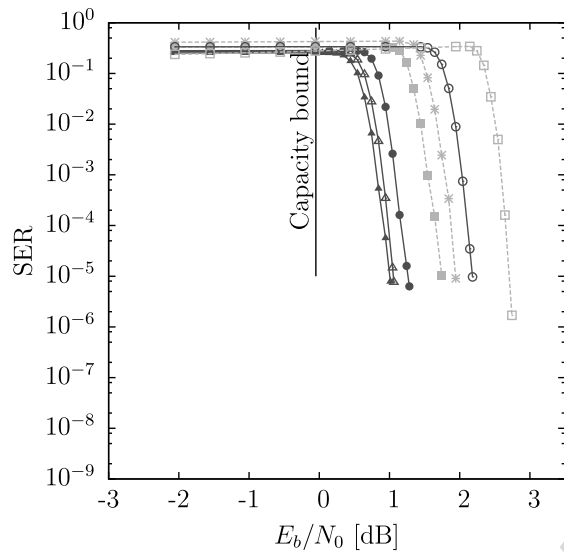

(a)

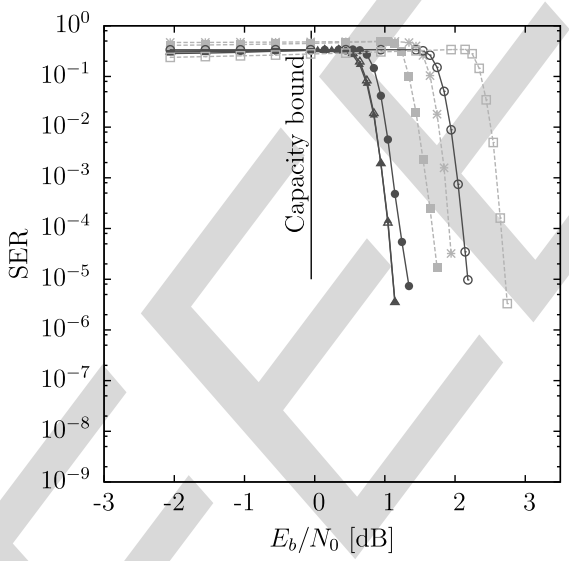

(b)

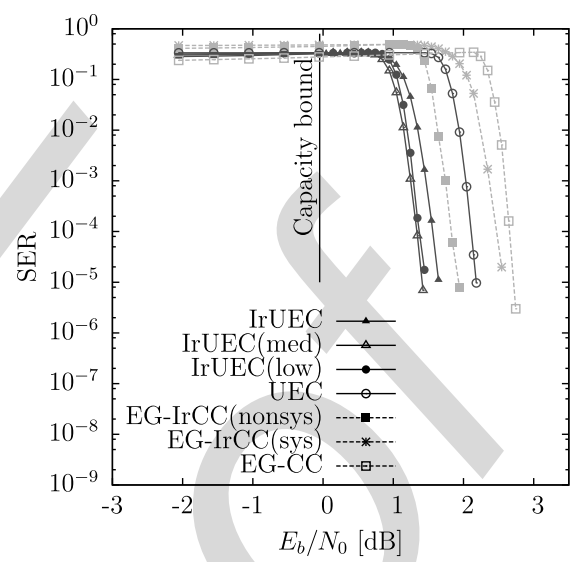

(c)

Fig. 10. SER performance for various arrangements of the proposed IrUEC-IrURC scheme of Fig. 1, the EG-IrCC-IrURC of Fig. 7, the Parallel IrUEC-IrURC scheme of Fig. 9, as well as the UEC-IrURC and the EG-IrURC schemes of [10], when conveying symbols obey a zeta distribution having the parameter $p_{1}=0.797$, and communicating over a QPSK-modulated uncorrelated narrowband Rayleigh fading channel having a range of $E_{\mathrm{b}} / N_{0}$ values. A complexity limit of (a) unlimited, (b) 10,000 and (c) 5,000 ACS operations per decoding iteration is imposed for decoding each of the bits in $\mathbf{z}$.

of separate UEC trellis encoders to encode the bit vector $\mathbf{y}$, in analogy with the structure of the EG-IrCC scheme. More specifically, the component UEC codes of the Parallel IrUEC encoder are selected from the five constituent codes provided in Table II, while the component UEC codes of the Parallel IrUEC decoder are extended to $r=10$ states. The irregular fractions employed by the Parallel IrUEC scheme are the same as those used in our proposed IrUEC scheme. However, in order for each component UEC trellis encoder to remain synchronized with the unary codewords in the bit vector $\mathbf{y}$, it is necessary for each component trellis to commence its encoding action from state $m_{0}=1$ and end at state $m_{b}=1$ or $m_{b}=2$. Owing to this, the subvectors of $\mathbf{y}$ input to each component UEC must comprise an integer number of complete unary codewords. The irregular coding fractions can only be controlled at the symbol level in the case of the parallel IrUEC scheme, rather than at the bit level, as in the proposed IrUEC scheme. Therefore, the corresponding EXIT chart of the parallel IrUEC scheme is not guaranteed to have an open tunnel, when the $E_{\mathrm{b}} / N_{0}$ value approaches the tunnel bound of Table III, hence resulting in a 866 degraded SER performance. However, if the frame length $a$ was 867 orders of magnitude higher, the difference between the symbol- 868 based and bit-based segmentations of the bit vector $\mathbf{y}$ would 869 become insignificantly small. As a result, a similar SER per- 870 formance may be expected for the parallel IrUEC scheme in 871 this case. In the following section, we will compare the perfor- 872 mances of the Parallel IrUEC and the proposed IrUEC schemes, 873 using different values for the frame length $a$.

\section{SER Results}

The SER performance of the IrUEC-IrURC, the EG- 876 IrCC(sys)-IrURC and the EG-IrCC(nonsys)-IrURC, UEC- 877 IrURC and EG-CC-IrURC schemes is characterised in Fig. 10. 878 In each case, the source symbol sequence $\mathbf{x}$ comprises $a=10^{4} 879$ symbols, the values of which obey a zeta distribution hav- 880 ing a parameter value of $p_{1}=0.797$. As shown above, the 881 parametrizations of the irregular codes in each scheme are 882 
designed to achieve the closest possible matching of EXIT charts, while giving the same overall effective throughput of $\eta=0.508 \mathrm{bit} / \mathrm{s} / \mathrm{Hz}$. Transmission is performed over a Graycoded QPSK-modulated uncorrelated narrowband Rayleigh fading channel, resulting in the DCMC capacity bound of $-0.05 \mathrm{~dB}$. We select two parametrizations of the schemes in [10] to create two of our four benchmarkers, namely the $r=4$-state UEC-IrURC and the $r=4$-state EG-CC-IrURC schemes. Note that the $r=4$-state EG-CC-IrURC scheme was found to outperform other parametrizations of the same scheme having higher number of states, owing to its superior EXIT chart matching accordingly. With the same effective throughput $\eta$, a fair comparison is provided between our proposed IrUEC-IrURC scheme and the four benchmarkers.

Note that the practical implementation of the time-variant IrTrellis used in our IrUEC-IrURC scheme follows the same principles as the parallel time-invariant trellises of the benchmarker schemes, such as the EG-IrCC-IrURC scheme and the regular UEC-IrURC scheme. Once the irregular coding fractions have been determined, the specific portions of message that should be encoded and decoded by the corresponding trellises are also determined. In both time-variant and parallel time-invariant trellises, the hardware is required to support different trellis structures, which may be implemented by appropriately changing the connections among the states of a single hardware implementation of a trellis. Although the proposed time-invariant trellis has some peculiarities at the interface between its different sections, these can also be implemented using the same hardware at either side of the interface. As an example platform for hardware implementation, the computation unit of [32] performs one ACS arithmetic operation per clock cycle, which are the fundamental operations used in BCJR decoders [18]. Therefore, the implementational complexity depends only on the computational complexity, as quantified per decoding iteration in Table III. Since a common computational complexity limit is used in our comparisons of the various schemes, they can be deemed to have the same implementational complexity. Although the routing and control of the proposed IrTrellis may be expected to be more complicated than in the parallel time-invariant trellises of the benchmarkers, it may be expected that the associated overhead is negligible compared to the overall implementational complexity.

As shown in Table III, our IrUEC-IrURC scheme imposes a complexity of 258 ACS operations per iteration per bit, when employing $r=10$ states for each component UEC code in the IrTrellis decoder. We also consider alternative parametrizations of our IrUEC-IrURC scheme, which employ an IrTrellis having fewer states, in order to achieve lower complexities. The IrUEC(med)-IrURC scheme relies on $r=6$ trellis states for different stages of the IrTrellis, which results in a total complexity of 192 ACS operations per iteration per bit. This matches that of the UEC-IrURC benchmarker. At the same time, the IrUEC(low)-IrURC scheme employs the minimal number of states for each stage of the IrTrellis, namely either $r=4$ states, as listed in Table II, hence resulting in a complexity of 157 ACS operations per iteration per bit.

During the simulation of each scheme, we recorded both the SER and the complexity incurred after each decoding iteration, resulting in a 3D plot of SER versus $E_{\mathrm{b}} / N_{0}$ and ver- 941 sus complexity. Fig. 10 presents 2D plots of SER versus $E_{\mathrm{b}} / N_{0} 942$ relationship, which were obtained by slicing through these 3D 943 plots at a particular complexity. More specifically, we select the 944 complexity limits of 10,000 and 5,000 ACS operations per iter- 945 ation per bit in Fig. 10(b) and (c), respectively. Meanwhile, 946 Fig.10 (a) characterizes the SER performance achieved after 947 iterative decoding convergence, regardless of the complexity. $\quad 948$

As shown in Table III, the proposed IrUEC-IrURC scheme 949 has an area bound of $0.21 \mathrm{~dB}$, which is the $E_{\mathrm{b}} / N_{0}$ value where 950 the area $A_{\mathrm{o}}$ beneath the inverted IrUEC EXIT function equals 951 that beneath the IrURC EXIT function. Although the UEC- 952 IrURC benchmarker has a similar area bound of $E_{\mathrm{b}} / N_{0}=953$ $0.49 \mathrm{~dB}$, it has an inferior EXIT chart matching capability 954 owing to its employment of regular UEC constituent codes. By 955 contrast, the employment of two irregular codes in the proposed 956 IrUEC-IrURC scheme facilitates an open EXIT chart tunnel at 957 an $E_{\mathrm{b}} / N_{0}$ value of $0.3 \mathrm{~dB}$, which is $1.4 \mathrm{~dB}$ lower than the open 958 tunnel bound of the UEC-IrURC benchmarker. Note that the 959 area and tunnel bounds are degraded in the context of the lower 960 complexity versions of the proposed IrUEC-IrURC scheme, 961 which have fewer states in the IrTrellis. This may be explained 962 by the increased capacity loss encountered when the number 963 of UEC states is reduced [10]. Note however that even with a 964 reduced complexity, the proposed IrUEC-IrURC scheme tends 965 to exhibit superior area and tunnel bounds, when compared 966 to the EG-IrCC-IrURC and EG-CC-IrURC benchmarkers, as 967 shown in Table III. This may be attributed to the large capacity 968 loss that is associated with SSCC scheme [10]. 969

Fig. 10 demonstrates that our proposed IrUEC-IrURC 970 scheme has a superior SER performance compared to all other 971 benchmarkers, regardless of which complexity limit is selected 972 in this particular scenario. For example, as shown in Fig. 10(a), 973 our IrUEC-IrURC scheme facilitates operation within $0.4 \mathrm{~dB}$ of 974 the capacity bound, offering a $0.8 \mathrm{~dB}$ gain compared to the EG- 975 IrCC(nonsys)-IrURC scheme, which is the best-performing of 976 the SSCC benchmarkers. This is achieved without any increase 977 in transmission energy, bandwidth, transmit duration or decod- 978 ing complexity. Note that the EG-IrCC(nonsys)-IrURC bench- 979 marker offers a $0.9 \mathrm{~dB}$ gain over the EG-IrCC(sys)-IrURC 980 benchmarker, which is owing to the capacity loss that is asso- 981 ciated with systematic IrCC component codes. As expected, 982 the reduced complexity versions of the proposed IrUEC-IrURC 983 scheme exhibit a degraded SER performance. However, the 984 IrUEC(low)-IrURC scheme can be seen to offer up to $0.5 \mathrm{~dB} 985$ gain over the UEC-IrURC benchmarker, which has a close 986 decoding complexity per bit per iteration. Since the Parallel 987 IrUEC-IrURC scheme can only provide a symbol-level con- 988 trol of the irregular coding fractions, the EXIT chart tunnel is 989 not guaranteed to be open at low $E_{\mathrm{b}} / N_{0}$ values. As a result, 990 Fig. 11 shows that the Parallel IrUEC-IrURC scheme of Fig. 9991 performs relatively poorly compared to the proposed IrUEC- 992 IrURC scheme, particularly when the frame length has values 993 of $a=10^{2}$ and $a=10^{3}$ symbols. Note that this performance 994 gain offered by the proposed scheme is obtained without impos- 995 ing any additional decoding complexity and without requiring 996 any additional transmission-energy, -bandwidth, or -duration. 997 In analogy with Fig. 10(a), an additional set of SER results 998 


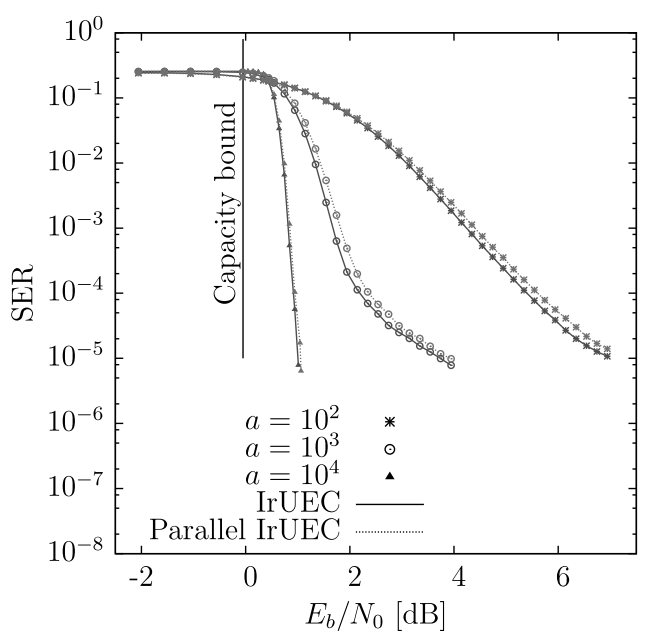

Fig. 11. SER performance for various frame lengths $a \in\left\{10^{2}, 10^{3}, 10^{4}\right\}$ of the proposed IrUEC-IrURC scheme of Fig. 1 and the Parallel IrUEC-IrURC scheme of Fig. 9, when conveying symbols obeying a zeta distribution having the parameter $p_{1}=0.797$, and communicating over a QPSK-modulated uncorrelated narrowband Rayleigh fading channel having a range of $E_{\mathrm{b}} / N_{0}$ values.

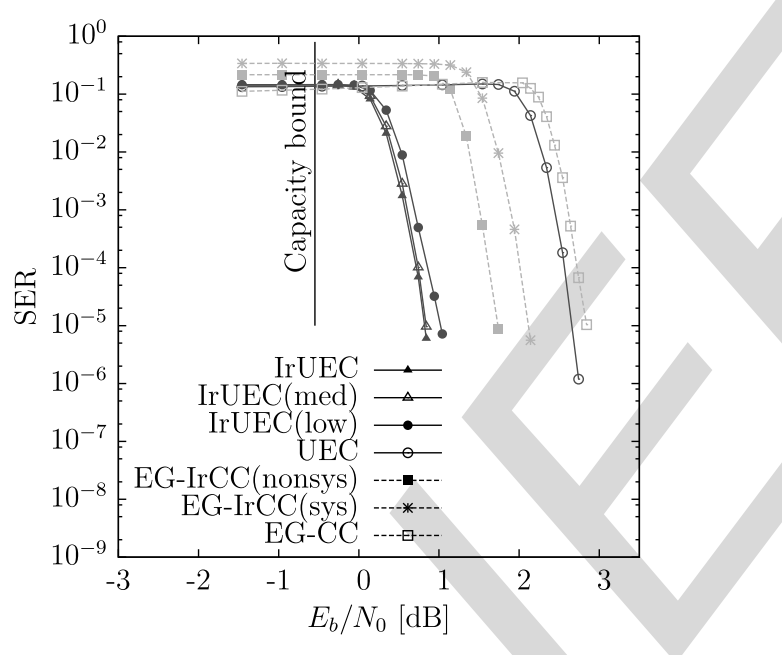

Fig. 12. SER performance for various arrangements of the proposed IrUECIrURC scheme of Fig. 1, the EG-IrCC-IrURC of Fig. 7, the Parallel IrUECIrURC scheme of Fig. 9, as well as the UEC-IrURC and the EG-IrURC schemes of [10], when conveying symbols obey a zeta distribution having the parameter $p_{1}=0.9$, and communicating over a QPSK-modulated uncorrelated narrowband Rayleigh fading channel having a range of $E_{\mathrm{b}} / N_{0}$ values. The complexity is unlimited for decoding each of the bits in $\mathbf{z}$. is provided in Fig. 12 for the various schemes considered, where the source symbols obey a zeta distribution having the parameter $p_{1}=0.9$, where the complexity is potentially unlimited. It can be seen that the proposed IrUEC-IrURC scheme also outperforms all other benchmarkers in this situation, offering a $1 \mathrm{~dB}$ gain compared to the EG-IrCC(nonsys)-IrURC scheme, which is the best-performing one of the set of SSCC benchmarkers.

Note that the performance gain of the proposed IrUECIrURC scheme is obtained by elaborately designing the IrUEC EXIT function, in order to create a narrow but marginally open EXIT chart tunnel at a low $E_{\mathrm{b}} / N_{0}$ value that is close to the area bound and capacity bound, as discussed in Section IV-B. Since the benchmarker schemes suffer from capacity loss which sep- 1012 arates their tunnel, area and capacity bounds, the performance 1013 gain of the proposed IrUEC-IrURC scheme depicted in Fig. 101014 and 12 may be expected in the general case, regardless of the 1015 specific source probability distribution and the parametrization 1016 of the scheme. As an additional benefit of the proposed IrUEC- 1017 IrURC scheme, a single bit error within a particular codeword 1018 can only result in splitting it into two codewords, or into merg- 1019 ing it with the next codeword, since every unary codeword 1020 contains only a single 0 . Fortunately, the decoding of the other 1021 unary codewords will be unaffected. Owing to this, a single bit 1022 error in the IrUEC-IrURC scheme can only cause a Levenshtein 1023 distance [33] of 2, hence preventing error propagation. By con- 1024 trast, in the EG-based benchmarkers, a single bit error can cause 1025 error propagation, resulting in a Levenshtein distance that is 1026 bounded only by the length of the message.

\section{CONCLUSIONS}

1028

In this paper, we have proposed a novel near-capacity JSCC 1029 scheme, which we refer to as the IrUEC code. Like the regular 1030 UEC code of [10], this employs a unary code, but replaces the 1031 UEC's trellis code with a novel IrTrellis code. Unlike a con- 1032 ventional irregular code, the IrTrellis code operates on the basis 1033 of a single amalgamated irregular trellis, rather than a number 1034 of separate trellises. Our results demonstrated that this single 1035 amalgamated trellis offers gains of up to $0.2 \mathrm{~dB}$ over the use 1036 of separate trellises, without imposing any increase in trans- 1037 mission energy, bandwidth, latency or decoding complexity. By 1038 characterizing the free distance property of the UEC trellis, we 1039 have selected a suite of UEC codes having a wide variety of 1040 EXIT chart shapes for the component codes of our IrUEC code. 1041 We concatenated the proposed IrUEC code with an IrURC code 1042 in Fig. 1 and introduced a new double-sided EXIT chart match- 1043 ing algorithm. On the one hand, the component UEC codes 1044 having a wide variety of EXIT chart shapes provide a great 1045 design freedom of the IrUEC EXIT chart. On the other hand, the 1046 novel double-sided EXIT chart matching algorithm utilize this 1047 design freedom sufficiently, in order to parametrize the IrUEC- 1048 IrURC scheme for creating a narrow but marginally open EXIT 1049 chart tunnel at a low $E_{\mathrm{b}} / N_{0}$ value that is close the area bound 1050 and the capacity bound. As a result, near-capacity operation 1051 is facilitated at $E_{\mathrm{b}} / N_{0}$ values that are within $0.4 \mathrm{~dB}$ of the 1052 DCMC capacity bound, when achieving an effective throughput 1053 of $\eta=0.508 \mathrm{bit} / \mathrm{s} / \mathrm{Hz}$ and employing (QPSK) for transmission 1054 over an uncorrelated narrowband Rayleigh fading channel. This 1055 corresponds to a gain of $0.8 \mathrm{~dB}$ compared to the best of several 1056 SSCC benchmarkers, which is achieved without any increase in 1057 transmission energy, bandwidth, transmit duration or decoding 1058 complexity.

\section{REFERENCES}

[1] J. Zou, H. Xiong, C. Li, R. Zhang, and Z. He, "Lifetime and distor- 1061 tion optimization with joint source/channel rate adaptation and network 1062 coding-based error control in wireless video sensor networks," IEEE 1063 Trans. Veh. Technol., vol. 60, no. 3, pp. 1182-1194, Mar. 2011.

[2] Y. Huo, C. Zhu, and L. Hanzo, "Spatio-temporal iterative source-channel 1065 decoding aided video transmission," IEEE Trans. Veh. Technol., vol. 62, 1066 no. 4, pp. 1597-1609, May 2013. 
1068

1069

1070

1071

1072

1073

1074

1075

1076

1077

1078

1079

1080

1081

1082

1083

1084

1085

1086

1087

1088

1089

1090

1091

1092

1093

1094

1095

1096

1097

1098

1099

1100

1101

1102

1103

1104

1105

1106

1107

1108

1109

1110

1111

1112

1113

1114

1115

1116

1117

1118

1119

1120

1121

1122

1123

1124

1125

1126

1127

1128

1129

1130

1131

1132

1133

1134

1135

1136

1137

1138

1139

1140
[3] N. S. Othman, M. El-Hajjar, O. Alamri, S. X. Ng, and L. Hanzo, "Iterative AMR-WB source and channel decoding using differential space-time spreading-assisted sphere-packing modulation," IEEE Trans. Veh. Technol., vol. 58, no. 1, pp. 484-490, Jan. 2009.

[4] C. E. Shannon, "The mathematical theory of communication," Bell Syst. Tech. J., vol. 27, pp. 379-423, Jul. 1948.

[5] B. Ryabko and J. Rissanen, "Fast adaptive arithmetic code for large alphabet sources with asymmetrical distributions," in Proc. IEEE Int. Symp. Inf. Theory, 2002, p. 319.

[6] J. Ziv and A. Lempel, "Compression of individual sequences via variable rate coding," IEEE Trans. Inf. Theory, vol. 24, no. 5, pp. 530-536, Sep. 1978.

[7] P. Elias, "Universal codeword sets and representations of the integers," IEEE Trans. Inf. Theory, vol. 21, no. 2, pp. 194-203, Mar. 1975.

[8] Advanced Video Coding for Generic Audiovisual Services, ITU-T Std. H.264, Mar. 2005.

[9] J. L. Massey, "Joint source and channel coding," in Proc. Commun. Syst. Random Process Theory, Dec. 1978, pp. 279-293.

[10] R. G. Maunder, W. Zhang, T. Wang, and L. Hanzo, "A unary error correction code for the near-capacity joint source and channel coding of symbol values from an infinite set," IEEE Trans. Commun., vol. 61, no. 5, pp. 1977-1987, May 2013

[11] N. L. Johnson, A. W. Kemp, and S. Kotz, Univariate Discrete Distributions. Hoboken, NJ, USA: Wiley, 2005.

[12] Y. Takishima, M. Wada, and H. Murakami, "Reversible variable length codes," IEEE Trans. Commun., vol. 43, no. 234, pp. 158-162, Feb. 1995.

[13] V. Buttigieg and P. G. Farrell, "Variable-length error-correcting codes," IEE Proc. Commun., vol. 147, no. 4, pp. 211-215, Aug. 2000.

[14] R. G. Maunder and L. Hanzo, "Near-capacity irregular variable length coding and irregular unity rate coding," IEEE Trans. Wireless Commun., vol. 8, no. 11, pp. 5500-5507, Nov. 2009.

[15] M. Tüchler, "Design of serially concatenated systems depending on the block length," IEEE Trans. Commun., vol. 52, no. 2, pp. 209-218, Feb. 2004

[16] R. G. Maunder and L. Hanzo, "Genetic algorithm aided design of component codes for irregular variable length coding," IEEE Trans. Commun., vol. 57, no. 5, pp. 1290-1297, May 2009.

[17] A. Ashikhmin, G. Kramer, and S. ten Brink, "Code rate and the area under extrinsic information transfer curves," in Proc. IEEE Int. Symp. Inf. Theory, Lausanne, Switzerland, Jun. 2002, p. 115.

[18] W. Zhang, R. G. Maunder, and L. Hanzo, "On the complexity of unary error correction codes for the near-capacity transmission of symbol values from an infinite set," in Proc. IEEE Wireless Commun. Netw. Conf., Apr. 2013, pp. 2795-2800.

[19] T. Wang, W. Zhang, R. G. Maunder, and L. Hanzo, "Near-capacity joint source and channel coding of symbol values from an infinite source set using Elias gamma error correction codes," IEEE Trans. Commun., vol. 62, no. 1, pp. 280-292, Jan. 2014

[20] L. Hanzo, R. G. Maunder, J. Wang, and L.-L. Yang, Near-Capacity Variable Length Coding. Hoboken, NJ, USA: Wiley, 2010.

[21] S. Benedetto and G. Montorsi, "Iterative decoding of serially concatenated convolutional codes," Electron. Lett., vol. 32, no. 13, pp. 11861188, Jun. 1996

[22] L. Bahl, J. Cocke, F. Jelinek, and J. Raviv, "Optimal decoding of linear codes for minimizing symbol error rate," IEEE Trans. Inf. Theory, vol. 20 no. 2, pp. 284-287, Mar. 1974

[23] R. G. Maunder and L. Hanzo, "Iterative decoding convergence and termination of serially concatenated codes," IEEE Trans. Veh. Technol., vol. 59, no. 1, pp. 216-224, Jan. 2010.

[24] J. Kliewer, N. Goertz, and A. Mertins, "Iterative source-channel decoding with Markov random field source models," IEEE Trans. Signal Process., vol. 54, no. 10, pp. 3688-3701, Oct. 2006.

[25] D. Divsalar, H. Jin, and R. J. McEliece, "Coding theorems for turbo like codes," in Proc. 36th Allerton Conf. Commun. Control Comput., Allerton House, IA, USA, Sep. 1998, pp. 201-210.

[26] A. Diallo, C. Weidmann, and M. Kieffer, "Efficient computation and optimization of the free distance of variable-length finite-state joint sourcechannel codes," IEEE Trans. Commun., vol. 59, no. 4, pp. 1043-1052, Apr. 2011.

[27] S. ten Brink, "Convergence behavior of iteratively decoded parallel concatenated codes," IEEE Trans. Commun., vol. 49, no. 10, pp. 1727-1737, Oct. 2001.
[28] M. Tüchler and J. Hagenauer, "EXIT charts of irregular codes," in Proc. 1141 Conf. Inf. Sci. Syst., Princeton, NJ, USA, Mar. 2002, pp. 748-753.

[29] L. Hanzo, T. H. Liew, B. L. Yeap, R. Y. S. Tee, and S. X. Ng, Turbo 1143 Coding, Turbo Equalisation and Space-Time Coding: EXIT-Chart Aided 1144 Near-Capacity Designs for Wireless Channels. Hoboken, NJ, USA: 1145 Wiley, 2010.

[30] J. Kliewer, A. Huebner, and D. J. Costello, "On the achievable extrinsic 1147 information of inner decoders in serial concatenation," in Proc. IEEE Int. 1148 Symp. Inf. Theory, Seattle, WA, USA, Jul. 2006, pp. 2680-2684. 1149

[31] P. Frenger, P. Orten, and T. Ottosson, "Convolutional codes with optimum 1150 distance spectrum," IEEE Commun. Lett., vol. 3, no. 11, pp. 317-319, 1151 Nov. 1999.

1152

[32] L. Li, R. G. Maunder, B. M. Al-Hashimi, and L. Hanzo, "A low- 1153 complexity turbo decoder architecture for energy-efficient wireless sensor 1154 networks," IEEE Trans. Very Large Scale Integr. (VLSI) Syst., vol. 21, 1155 no. 1, pp. 14-22, Jan. 2013

[33] D. Sankoff and J. B. Kruskal, Time Warps, String Edits, and 1157 Macromolecules: The Theory and Practice of Sequence Comparison. 1158 Reading, MA, USA: Addison-Wesley, 1983.

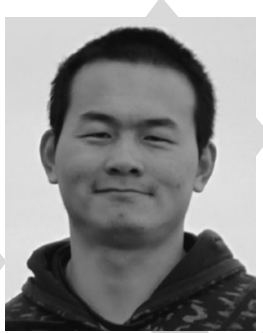

Wenbo Zhang (S'14) received the M.E. degree in 1160 information and communication engineering from 1161 the University of Beijing University of Posts and 1162 Telecommunications (BUPT), Beijing, China, in 1163 2011. Currently, he is pursuing the Ph.D. degree 1164 at the Communications Research Group, School of 1165 Electronics and Computer Science, University of 1166 Southampton, Southampton, U.K. His research inter- 1167 ests include joint source/channel coding and variable 1168 length coding.

1169

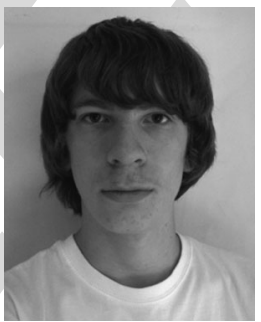

Matthew F. Brejza received the B.Eng. degree 1170 (first class Hons.) in electronic engineering from the 1171 University of Southampton, Southampton, U.K., in 1172 2012, and is currently pursuing the Ph.D. degree 1173 at the Communications Research Group, School of 1174 Electronics and Computer Science, University of 1175 Southampton. His research interests include flexible 1176 hardware implementation, channel coding and their 1177 applications in low power data communications.

1178

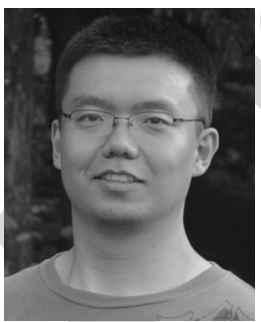

Tao Wang received the B.S. degree in informa- 1179 tion engineering from the University of Science 1180 and Technology of Beijing (USTB), Beijing, China, 1181 and the M.Sc. degree in communication from 1182 University of Southampton, Southampton, U.K., in 1183 2006 and 2008, respectively, and is currently pursu- 1184 ing the Ph.D. degree at the Communications Research 1185 Group, School of Electronics and Computer Science, 1186 University of Southampton. His research interests 1187 include joint source/channel coding and distributed 1188 video coding.

1189

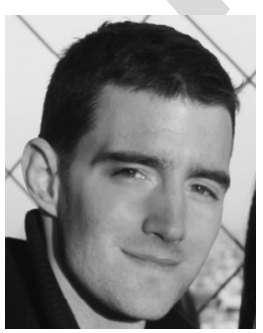

Robert G. Maunder (SM'12) received the B.Eng. 1190 degree (first class Hons.) in electronic engineering 1191 and the Ph.D. degree in wireless communications, 1192 in 2003 and 2007, respectively. He has authored a 1193 number of IEEE papers in his research interest areas. 1194 His research interests include joint source/channel 1195 coding, iterative decoding, irregular coding, and mod- 1196 ulation techniques. He received a lectureship in 1197 December 2007. 


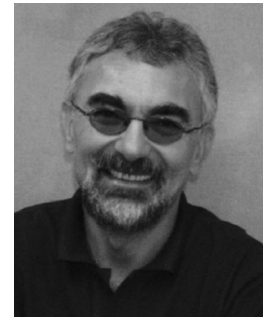

Lajos Hanzo (F'04) received the degree in electronics and the doctorate degree, in 1976 and 1983, respectively. During his 38-year career in telecommunications, he has held various research and academic posts in Hungary, Germany, and the U.K. Since 1986, he has been with the School of Electronics and Computer Science, University of Southampton, Southampton, U.K., where he holds the Chair in telecommunications. He has coauthored 20 Wiley/IEEE Press books on mobile radio communications totalling in excess of 10000 pages, authored more than 1500 research entries at the IEEE Xplore, acted both as TPC and General Chair of the IEEE conferences, presented keynote lectures and has been awarded a number of distinctions. Currently, he is directing a 60strong academic research team, working on a range of research projects in the field of wireless multimedia communications sponsored by industry, the Engineering and Physical Sciences Research Council (EPSRC), U.K., the European Research Council's Advanced Fellow Grant, and the Royal Society's Wolfson Research Merit Award. He is a Governor of the IEEE VTS. From 2008 to 2012, he was the Editor-in-Chief of the IEEE Press and also a Chaired Professor at Tsinghua University, Beijing, China. His research is funded by the European Research Council's Senior Research Fellow Grant. Lajos has more than 22000 citations. In 2009, he was was the recipient of an Honorary Doctorate by the Technical University of Budapest, while in 2015, by the University of Edinburgh. 